\title{
Polarization of the Cosmic Background Radiation
}

\author{
Philip Michael Lubin \\ Space Sciences Laboratory and Lawrence Berkeley Laboratory \\ University of California at Berkeley \\ Berkeley, California 94720
}

\begin{abstract}
This thesis discusses the results and technique of a measurement of the linear polarization of the Cosmic Background Radiation. The ground-based experiment utilizes a single horn ( $7^{\circ}$ beam width) Dicke-type microwave polarimeter operating at $33 \mathrm{GHz}(9.1 \mathrm{~mm})$. Data taken between May 1978 and February 1980 from both the northern hemisphere (Berkeley $I_{-a t}-38^{\circ} \mathrm{N}$ ) and the southern hemisphere (Lima Lat. - $12^{\circ} \mathrm{S}$ ) show the rudiation to be essentially unpolarized over all areas surveyed. For the $38^{\circ}$ declination data the $95 \%$ confidence level limit on a linearly polarized component is $\mathbf{0 . 3} \mathbf{m K}$ for the average and 12 and 24 hour periods. Fitting all date gives the $95 \%$ confidence level limit on $\mathbf{x}$ linearly polarized component of $0.3 \mathrm{mK}$ for spherical hamonics through third order. Constraints on various cosmological models are discussed in light of these limits.

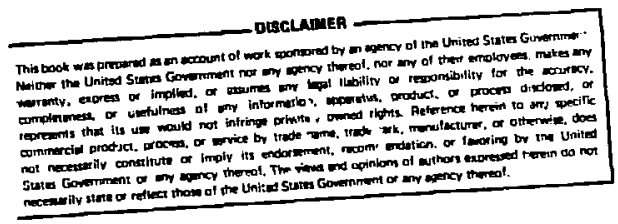


Table of Contents

Chapter I - Cosmology and the Cosmlc Background Radiation

I.I Introduction

1.2 Big Bang Cosmology 1

1.3 Current Knowledge of the Cosmic Brekground Radiation 2

I.4 Anisotroples 3

1.5 Polarization - Causes and Theory 6

1.6 Prevlous Mcarjiements 9

Chapter II - Theory of Measurement

11.1 Optical Analog 12

11.2 Stokes Parameters and Antenna Temperature 12

Chapter III - Experimental Technique

$\begin{array}{ll}\text { III.1 Apparatus Description } & 17\end{array}$

$\begin{array}{ll}\text { III.2 Mlerowave Radlometer } & 17\end{array}$

$\begin{array}{ll}\text { III.3 Thermal Regulation } & 20\end{array}$

$\begin{array}{ll}\text { III.4 Callbration } & 21\end{array}$

Chapter IV - Data Acquisition

IV.1 Data Recording $\quad 24$

IV.2 Data Taking * $\quad 24$ 
Chapter V - Data Reduction and Analysis

V.1 Data Reduction

V.2 Analysis Algorlthm

29

V.3 Data Deletion

32

Chapter VI - Data and Fits

V1.1 Data

V1.2 Polarization Limits at Each Eeclination

VI.3 Spherical Harmonic Fits

V1.4 Comparison to Previous Results

Chapter VII - Astrophysical Interpretation

VII.1 Limits on Anisotropic Models

VII.2 Comparison to Intensity Measurements

Appendix B - Microwave Circuit

B.1 General Description

$\begin{array}{ll}\text { B.3 Faraday Rotation Switch } & 60\end{array}$

B.4 Recelver 
Appendix C - Systematic Error Analysis

C.1 Introduction

C.2 Microwave Front End

C.3 Recelver and Post Detection Electronles

C.4 Atmospheric Fluctuations 68

\section{Appendix D - Backgrounds}

D.1 Introduction

D.2 Galactic and Extragalactic

D.3 Solar System Sources 74

D.3.1 Satellites 74

D.3.2 Dust

D.4 Atmosphere

D.5 Terrestrial Magnetic Field Effects

D.6 Depolarization Processes 


\section{List of Figures}

I.1 Cosmic Background Radiation Spectrum

1.2 Measured First Order Anisotropy'

1.3 Expansion Anisotropy and Resulting Polarization Pattern 10

11.1 Conversion of Stokes Parameter to Magnitude and Angle I? $^{\text {? }}$

$\begin{array}{ll}\text { II.2 Stokes Parameters } Q \text { and } U & 16\end{array}$

$\begin{array}{ll}\text { III.I Polarimeter Schematic } & 18\end{array}$

$\begin{array}{ll}\text { JI!.2 Radiometer System } & 19\end{array}$

$\begin{array}{lr}\text { III.3 Calibration } & 23\end{array}$

$\begin{array}{ll}\text { IV.1 Sky Coverage } & 27\end{array}$

$\begin{array}{lll}\text { V.1 RMS Fluctuation versus Integration Time } & 30\end{array}$

V.2 Data Analysis Agorithm 31

$\begin{array}{lr}\text { VI.1 Plot of Data } & 40\end{array}$

A.1 Wotbble Amplitude versus DC Level

$\begin{array}{ll}\text { B.1 Microwave Circuit } & 57\end{array}$

$\begin{array}{ll}\text { B.2a Measured Antenna Patterns } & 58\end{array}$

B.2b Measured Antenna Pattems with Ground Shield 59

$\begin{array}{llr}\text { B.3 Polarization Switch } & 61\end{array}$

D.1 Extimated Galactic : nchrotron Radiation at $33 \mathbf{~ G H z}$

$\begin{array}{ll}\text { D.2 Estimated Polarized Galactic Emission at } 33 \mathrm{GHz} & 73\end{array}$

E.1 Polarization Basis Vectors and Axisymmetric Coordinate Systam 79

E.2 Polarization and Intensity Anisotropy in an Axisymmetric Univere 82 


\section{List of Tables}

I.1 Possible Causes of Anisotropy and Polarization

I.2 Measured Limits on Linear Polarization

IV.1 Information Recorded on Tape

V.1 Statistical Tests of Datn

VI.1 Data

V1.2 Individual Declination Fits

V1.3 Spherical Harmonic Fits

VI.4 Fit to Anisotropic Axisymmetric Model (Rees)

V1.5 Results of Previous Measurements

VII.I Model Constraints from Polarization Data

VI1.2 Comparison to Intensity Measurements

VII.3 Comparison of Polarization and intensity

A.1 Wobble Correction

C.1 Polarimeter System Performance Parameters

C.2 Temperature Stability of Components

D.1 Systematic Errors: Causes and Solutions

D.2 Faraday Rotation and Depolarization 


\section{Chapter I - Cosmology and the Cosmic Background Radiation}

\subsection{Introductlon}

We are living in an era which is revolutionizing our understanding of the universe. This has been achieved primarily by the development of a viable gravitational theory (Einstein's General Relativity) combined with our increased understandins of particle physics. This union of large and small has led to a situation where predictions are many but relevant observations are few.

A basic tenant in all physics is symmetry. Its presence or absence in nature often yields information far beyond the immediate observation. Ultimately the experiment described in this thesis is concerned with a large scale symmetry in the radiation field (Cosmic Background Radiation). This in turn allows us to infer knowledge of the symmetry in the matter distribution of the universe, and beyond that, tells us something of the processes that took place in the early universe.

\section{I.2 Big Bang Cosmology}

There are currently several theories describing the evolution of the universe. The one which is most consistent with prestent dato is the so-called "Hot Big Bang Theory" (Harrison, 1973). Briefly it says the universe evolved explosively from a hot dense initial state. The origin of this initial state is unknown. To account for the presently observed helium abundance (approximately 25\% by mass), an energetic photon gas is postulated to exiat in the early universe. This allows suficient photodissociation of the forming deuterium to prevent all the protons and neutrons from producing helium and teavier elements. Rapid thermalization of the pholons occurs because of the numerous scatterings. It is believed that the remnant of this radiation is observed today as the Cosmic Background Raciation (CBR). In is remarkable that the existence of this radiation was prejicted almost two decades before it was discovered in 
1965 (Alpher, Bethe \& Gamow, 1948).

Several minutes after the beginning, the helium to hydrogen ratio is frozen out and essentially all element production stops. For roughly a million years the universe expands adiabatically and uneventfully, until the temperature has cooled sufficiently to allow the electrons to combine witi nuclei to form neutral monatomic matter. This occurs approximately at a temperature of $4000^{\circ} \mathrm{K}$ (Peebles, 1968) or a redshift of $Z-1500$ (redshift being defined so that $1+Z$ is just the ratio of the present linear scale size to the past scale size). Since the expansion is essentially adiabatic, the photon temperature drops inversely as the linear scale size, analogous to classical adiabatic expansion of a photon gas.

This is called the "era of recombination" or "decoupling" since the photons no longer effectively interact with the matter after this time. This is because the cross section for interaction between matter and radiation drops from the Thomson cross section before recombination to the Rayleigh cross section (scattering off bound electrons) after recombination. From the time of decoupling $Z-1500$ to the time of galaxy formation, mattet and radiation evolve independently. Whether matter is re-ionized and, thus ggain interacts with the radiation during galaxy formation is uncertain. The time scale for this entire sequence is thought to be of the order of 10 to 20 billion years.

\subsection{Current Knowledge of the Cosmlc Background Radiatlon}

The Cosmic Background Radiation (CBR), discovered in 1965 by Penzias and Wilson (Penzias \& Wilson, 1965), has had profound implications regarding our understanding of the universe as a whole. Thought to have been emitted within minutes of the beginning of the universe, it is the culmination of billions of years of evolution and $x$ unique probe into the past.

As a radiation field, it can be uniquely characterized by its electric field $\vec{E}(\vec{x}, t)$ at each point in space and time. Equivalently by Fourier analysis, it can be represented in momentum space by its transform $\vec{E}(\bar{\kappa}, \omega)$ 
Physically, at a fixed point in space, we can specify the field by its:

(1) Spectrum $\vec{E}(\bar{\kappa}, \omega)$

(2) Angular distribution $\bar{E}(\overrightarrow{\boldsymbol{x}}, \boldsymbol{\omega})$ and,

(3) Polarization state $\vec{E}(\vec{\kappa}, \omega)$.

These three features then uniquely characterize the radiation at a particular point in space.

Our current understanding of the spectrum is that it qualitatively is a blackbody of characteristic temperature about $3^{\circ} \mathrm{K}$ with a possible deviation from a purely Planckian spectrum (Woody \& Richards 1979). Figure 1.1 shows the current measurements of the spectrum. The angular distribution of the radiation is nearly isotropic, with a deviaticn of amplitude -3.5 $\mathrm{m}^{\circ} \mathrm{K}$, interpreted as being due to the motion of the earth through the radiation (Corey \& Wilkinson, 1976; Smoot, Gorenstein \& Muller, 1977). After removal of this "first order anisotropy no residual anisotropy is seen with a $95 \%$ confidence level of $1 \mathrm{~m}^{\circ} \mathrm{K}$ for quadrupole terms (Smoot \& Lubin, 1979; Gorenstein \& Smoot, 1980). Figure 1.2 shows the measured first ordf isotropy. The current knowledge of the polarization state of the radiation is the subject of this thesis, but to spare the reader undue anxiety, no evidence for linear polarization is seen (Cademi et al., 1978; Lubin \& Smoot, 1979; Nanos, 1979).

A decade and a hallf after its discovery, our knowledge of this assumed primordial radiation can be summarized as the radiation being approximately blackbody, nearly isotropic, and essentially unpolarized. In the next decade our understanding of the Cosmic Background Radiation should increase substantially due to vigorous interest, particularly with the launch of the Cosmic Background Explorer (COBE) satellite dedicated to its study.

\subsection{Anlsotroples}

There are two basic classes of anisotropies, those intrinsic to the radiation itself and those which are extrinsic in origin. The latter are typified by the first order anisotropy caused by the motion of the observer through the radiation. This is what Peebles calls the "New Aether 
FIgure I.1 - Cosmic Background Radiation Spectrum

( Hoody \& Richardo 1979 )

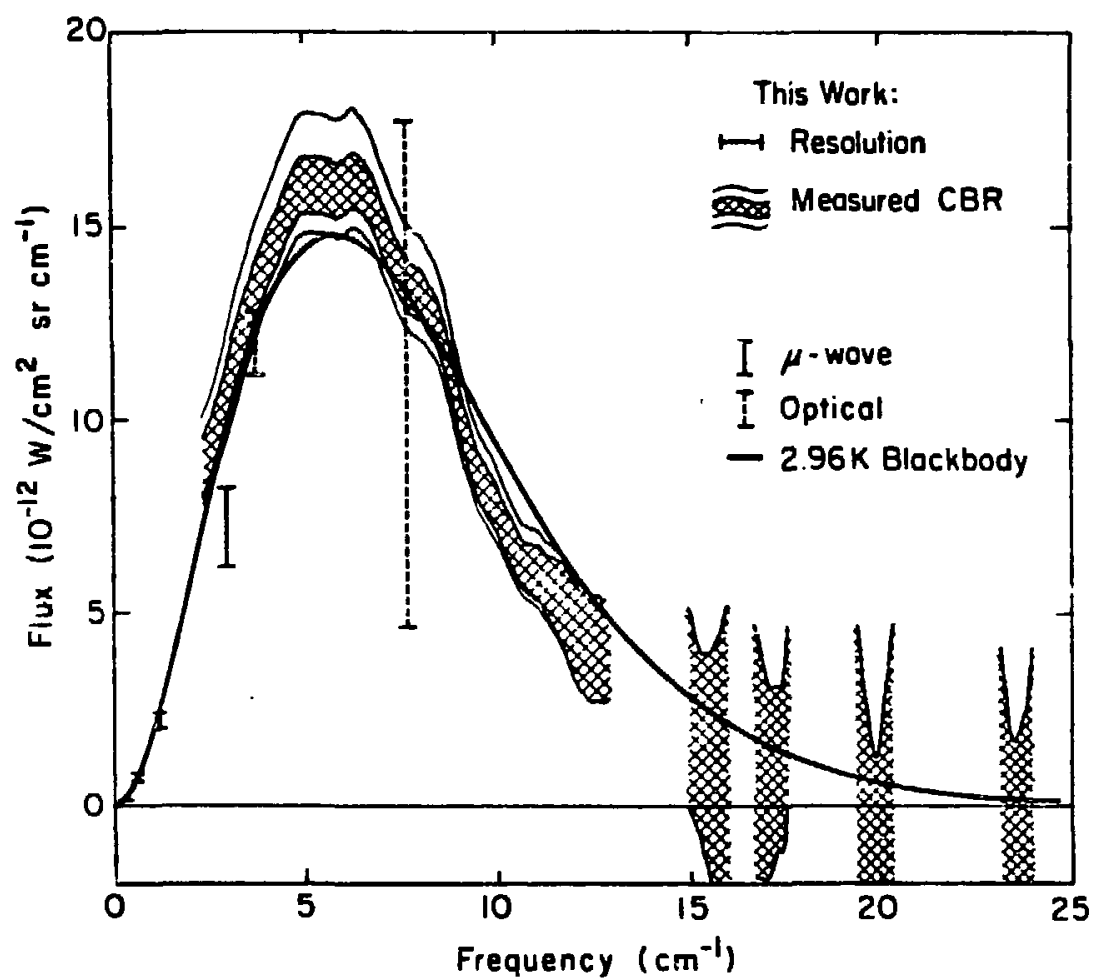

XBL 789-5876B 
Figure I.2 - Messured First Order Anlsotropy

( data from Smoot \& Lubin 1972, Corenstein a Smoot 1080)

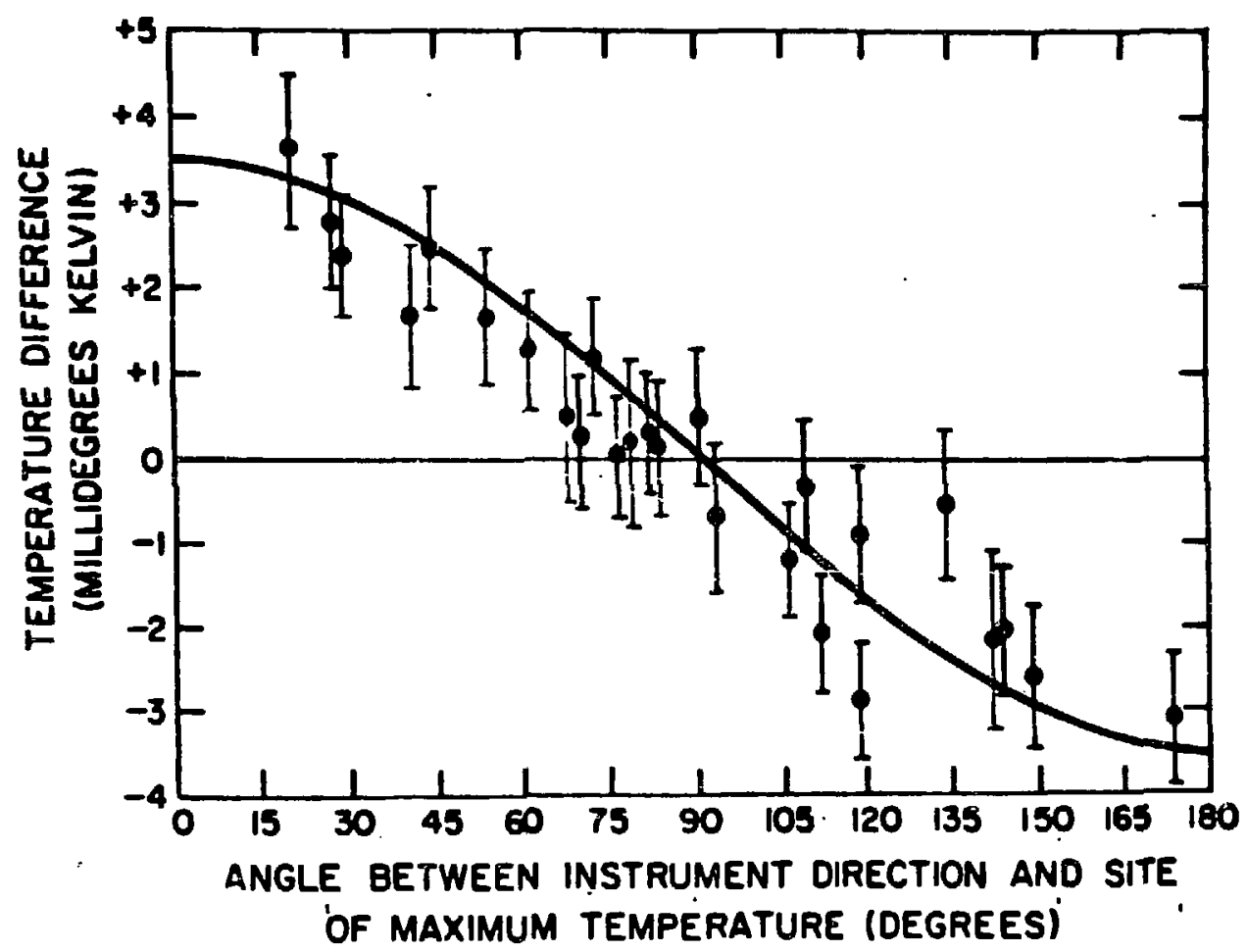


Drift" (Peebles, 1971). Also in this category are local density inhomogeneities which through their increased (or decreased) gravitational attraction cause deviations from the local co-moving frame (Hubble flow), and again cause a motional induced anisotropy. These extrinsic types of anisotropies are generally not accompanied by a net polarization.

Any intrinsic anisotropy, such as some of those listed in Table I.1 is accompanied by a net polarization. In general, intrinsic anisotropies are expected, though the level is uncertain. From causality arguments alone, anisotropies should arise because parts of the universe have been out of the light cone (out of communication) with other parts. In simple models anisotropy is expected on an angular scale size characterized by $\theta_{r}=4.2^{\circ} \sqrt{q_{0}}$ where $q_{0}$ is the deceleration parameter (Weinberg, 1972). If $q_{0}=0.5$ (minimum needed for closed universe), then $\theta$, $-3^{\circ}$. If the universe were rotating, tiren an intrinsic anisotropy would also be expected (Hawking, 1969). Thus, anisotropy measurements and hence, polarization measurements provide a test of :Aach's principle (Mach, 1893).

Anisotropic expansion of the universe is discussed more fully in Appendix E. Heuristically, this type of expansion causes an anisotropy because the universe expands more rapidly in some directions than in others, and thus, radiation is red shifted by differing amounts in difierent directions.

\subsection{Polarization - Causes and Theory}

The reasons for studying the polarization of the CBR are several. First, it is a basic characteristic of any electromagnetic radiation (interesting in its own right) and second, any intrinsic anisotropy of the CBR should be accompanied by a net polarization. That this second reason is physically plausible can be seen literally, by gazing outside on a clear day. Of common knowledge is the fact that the sky appears blue. Less discemible, but still noticeable to some well adapted human eyes (Shurcliff \& Ballard, 1962) and most bees (Waterman, 1955), is that the scattered sunlight is slightly linearly polarized. These two effects are due to the scattering of light from an anisotropic illuminator, namely the sun. The primary scattering mechanism is 
Table I.1 - Posstble Causes of Anisotropy and Polarization

\section{POSSIBLE CAUSES OF ANISOTROPY AND POLARIZATION IN THE $3^{\circ} \mathrm{K}$ COSMIC BACKGROUND RADIATION}

\begin{tabular}{l|c|c}
\multicolumn{1}{c}{ ANISOTROPY CAUSE } & TYPE & POLAFIZATION \\
\hline Motion of observer & LOCAL & NO \\
Rotation of universe & INTRINSIC & YES \\
$\begin{array}{l}\text { Long.wavelength gravity } \\
\text { waves }\end{array}$ & INTRINSIC & YES \\
$\begin{array}{l}\text { Anisotropic expansion } \\
\text { (Shear) }\end{array}$ & INTRINSIC & YES \\
$\begin{array}{l}\text { Density inhomogeneities } \\
\text { A) Primordial }\end{array}$ & INTRINSIC & YES \\
B) Local & LOCAL & NO \\
$\begin{array}{l}\text { Motion of source } \\
\begin{array}{l}\text { Transverse motion } \\
\text { of clusters }\end{array}\end{array}$ & INTRINSIC & YES \\
& LOCAL & YES
\end{tabular}


Rayleigh scattering (scattering by bound electrons). The biue results from the frequency dependence of the cross section, while the polarization results because the electron is accelerated by the scattering interaction and preferentially radiates with polarization perpendicular to the direction of illumination and observaticn.

In the cosmological case, the effect is precisely the same. If an anisotropy in intensity exists in the CBR, then the radiation acquires a net linear polarization by scattering off the : רatter. In principle any intrinsic anisotropy in intensity leads to a net linear polarization. Hence, studying the polarization properties of the radiation serves a dual purpose: (1) it measures possible inherent polarization that may exist, and (2) it provides a secondary means of searching for any intrinsic anisotropy in intensity.

Since scattering is the essential mechanism, the type of scattering involved is important. While the matter in the universe is a hot plasma, the primary interaction betweer jiation and matter is via Thomson scattering. This has a frequency independent total cross section of $\sigma_{T}=8 \pi / 3 r_{p}^{2}=0.67$ barns for $h \nu \ll m_{p} c^{2}$. After the nuatter has cooled sufficiently to combine (decoupling), the primary interaction between radiation and the neutral matter is Rayleigh scattering of the bound elecirons with a frequency dependent total cross section of $\sigma_{R}=\left(\frac{\nu}{\nu_{0}}\right)^{4} \sigma_{T}$ for $\nu \ll \nu_{0}$ where $h \nu_{0}$ is the binding energy. The matter in the universe is primarily hydrogen, so $h v_{0}-10^{\prime} \mathrm{ev}$ and $\nu_{0}-10^{16} \mathrm{~Hz}$. At our frequency of observation $y=3 \times 10^{10} \mathrm{~Hz}$, so at decoupling these same photons had a frequency of $5 \times 10^{13} \mathrm{~Hz}$. This is small conspared to $\nu_{0}, 50$ Rayleigh scattering after decoupling is negligible compared to Thomson scattering before. Actually Thomson scattering from residual ionization ( $0.01 \%)$ is thought to dominate ufter decoupling (Peebles 1968). In terms f the mean time between scatterings, the typical mean free path is $\mathbf{1 0 0}$ years before decoupling and a million years after for a critically dense universe. This is to be compared to the typical scale size of the universe at Gecoupling of less than a million years. So there are many scatterings before decoupling but typically less than one per photon after. 
As mentioned in the reivious section, intrinsic anisotropies are expected in the background radiation if for no other reason than the causality arguments given previously. Since this anisotropy is expected to have originated before the period of recombination, a net polarization is also expected by the reasons given above. Unfortunately, the level at which this anisotropy and hence, polarization is expected, is unknown. Rees (1968) originally suggested that an anisotropically expanding universe would generate a net polarization because of the anisotropy in the intensity of the CBR. Figure 1.3 shows the polarization pattern expected for two c ases of an axisymmetric expansion. This is discussed in detail in appendix E.

Additionally, the polarization measurements provids a check of the first order anisotropy seen in intensity. If the anisotropy is due to our motion, no net polarization is expected; however, if this first order anisotropy is intrinsic to the radiation itself in part or total, a net polarization could exist. So a null result tends to suppon the interpretation of the intensity anisotropy as being locally induced by our motion.

\section{I.6 Prevlous Measurements}

Since the suggestion by Rees (1968) that anisotropic expansion would yield a net linear polarization in the Background Radiation, little attention has been directed towards this method of searching for anisotropies. It is more straightforward to look for anisotropies in measurements of the intensity rather than in the polarization. However when it is realized that the polarization measurement is sensitive only to intrinsic anisotropies, it becomes an interesting test on its own merits. In their original paper on the discovery of the Background Radiation (Penzias \& Wilson, 1965), it is stated that the signal is "isotropic, unpolarized and free from seasonal variation". We now know that the radiation is slightly anisotropic by about one part in a thousand, due to our motion in the galaxy and the motion of our galaxy. We also believe the intensity to in fact be dependent on the seasons, to about a part in ten thousand, owing to the earth's motion around the sun. 
Figure I.3 - Expansion Anisotropy and Resulting Polarization Pattern

EXPANSION

ANISOTROPY
RESULTINS

POLARIZATION PATTERN ON SKY

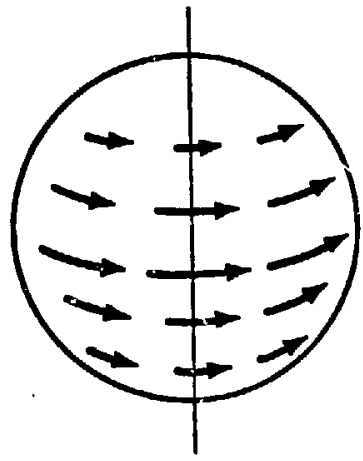

"Cigar" Universe

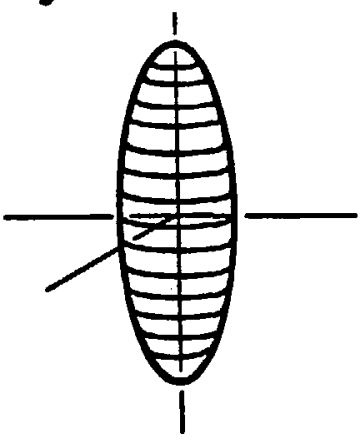

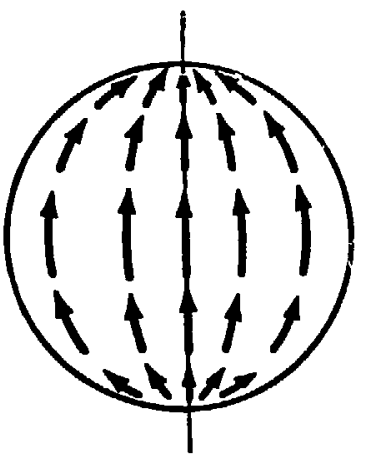

XBL791-184 
In 1972, George Nanos $(1974,1979)$ under Dave Wilkinson at Princeton, initiated an experiment to search for linear polarization with a null result. In addition Caderni et al (1978) reported no net linear polarization from an infrared balloon experiment. Unfortunately the balIoon flight was terminated prematurely, so only a small portion of the sky could be surveyed. Table 1.2 summarizes the previous measurements.

\begin{tabular}{|l|l|l|l|}
\hline \multicolumn{4}{|c|}{ Table I.2 } \\
\hline \multicolumn{2}{|c|}{ Measured Limits on Linear Polarization $95 \%$ Confidence Level } \\
Penzias and Wilson (1965) & 7.35 & \multicolumn{1}{|c|}{ Sky Coverage } & Limit \\
Nanos (1979) & 3.2 & scattered & $10 \%$ \\
Caderni etal (1978) & $0.05-0.3$ & $\begin{array}{l}\text { declination }-+40^{\circ} \\
\text { near galactic center }\end{array}$ & $0.05 \%$ \\
This work & 0.91 & $\begin{array}{l}11 \text { declinations } \\
-37^{\circ} \text { to }+63^{\circ}\end{array}$ & $0.006 \%$ \\
\hline
\end{tabular}




\section{Chapter II - Theory of Measurement}

\subsection{Optical Analog}

One can determine the polarization state of optical radiation by taking a linear polarizing sheet and rotating it about the line of sight. Any variation in intensity with angle will be indicative of linear polarization. Similarly, by using a quarter wave plate to convert circular polarization to linear polarization, the circular polarization state of the radiation can be determined.

The measurement in the microwave is very much analogoss. As a rotating polarizer, a switch (Faraday Rotation Switch) is used. It alternately selects orthogonal polarization states. the difference in power being indicative of net linear polarization. Rotating the instrument by $45^{\circ}$ allows both Stokes parameters $Q$ and $U$ to be measured. The connection between $Q, U$, and the magnitude, angle (of the polarization vector) is shown in Figure Il.1.

\section{II.2 Stokes Parameters and Antenna Temperature}

Monochromatic radiation can be uniquely described by four parameters:

(1) number of photons arriving

(2) number of right (or left) helicity photons

(3) phase angle

(4) fraction of randomly phased photons

These four parameters are conveniently reparameterized by the four Stokes parameters (Kraus, 1966; Chandrasekhar, 1950) I, Q, U, and V, which provide the following information:

I - total intensity

$Q$ - intensity in one linear polarization state minus the orthogonal state 
F1gure II.1 - Conversion of Stokes Parameters to Magnitude and Angle

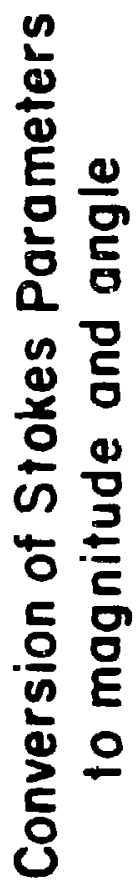

$$
\begin{aligned}
& \mathbf{d} \\
& \mathbf{v} \\
& \mathbf{0} \\
& \frac{1}{0} \\
& \frac{1}{\infty} \\
& x
\end{aligned}
$$
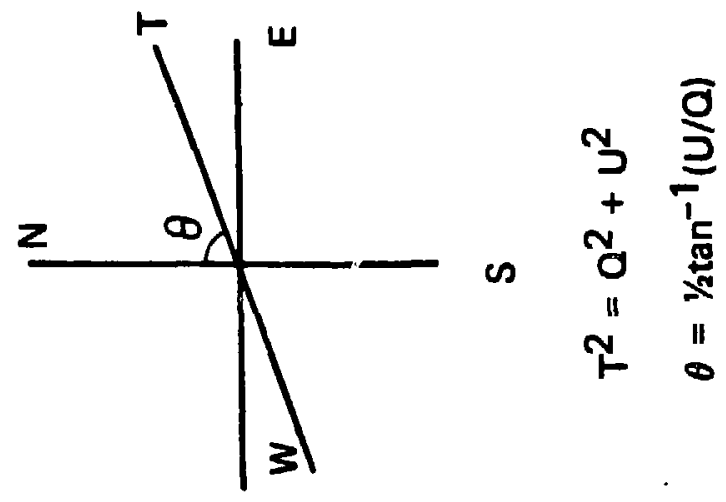
$U$ - same as $Q$ but rotated by $45^{\circ}$

v - left circular polerization intensity minus the right

For completely polarized radiation, $\mathrm{I}^{2}=\mathrm{Q}^{2}+\mathrm{U}^{2}+\mathrm{V}^{2}$ whereas for partially polarized radiation $\mathrm{I}^{2}>\mathrm{Q}^{2}+\mathrm{u}^{2}+\mathrm{v}^{2}$.

The purpose of this experiment is to determine $Q$ and $U$ for the CBR.

For black body radiation of temperature $T$, the flux $I$ is given by:

$$
I=\frac{2 h \mathrm{r}^{3}}{c^{2}} \frac{1}{e^{h / / \mathrm{L}}-1} \operatorname{erg} \mathrm{cm}^{-2} \sec ^{-1} \mathrm{si}^{-1} H_{z}^{-1}
$$

for hv $<<k T$ this reduces to:

$$
I=\frac{2 k T}{\lambda^{2}}
$$

This is the so-called Rayleigh Jeans limit.

For a given nux $1\left(\operatorname{ergs~} \mathrm{cm}^{-2} \mathrm{sec}^{-1} \mathrm{st}^{-1} \mathrm{~Hz}^{-1}\right)$, the Antenna Temperature $\mathrm{T}_{A}$ is defined such that, in the Rayleigh-Jeans limit, the flux produced by a black body of temperature $T_{A}$ would produce the given fux 1. Because microwave radiometers measure flux, it is convenient to define an equivalent temperature $T_{A}$ as:

$$
T_{1}=\frac{\wedge^{2}}{2 k} l
$$

Using (II.1) for I gives:

$$
T_{A}=\frac{x}{e^{x}-1} T, \quad x=\frac{h v}{k T}
$$

Also

$$
\frac{d T_{A}}{d T}-\frac{x^{2} e^{x}}{\left(e^{x}-1\right)^{2}}
$$


The antenna temperature at $\nu=33 \mathrm{GHz}$ for $\mathrm{T}=2.7 \mathrm{~K}$ is:

$$
T_{A}=2.0 \mathrm{~K} \text { while } \quad \frac{d T_{A}}{d T}=0.98
$$

In terms of antenna temperature $Q$ and $U$ are defined as follows:

$$
\begin{aligned}
& Q=T_{N S}-T_{E W} \\
& U-T_{N W . S E}-T_{N E . S W}
\end{aligned}
$$

where:

$$
\begin{aligned}
& \mathrm{T}_{\mathrm{NS}} \text { - antenna temperature of radiation polarized along } \\
& \text { the north-south direction } \\
& \mathrm{T}_{\mathrm{EW}} \text { - antenna temperature of radiation polarized along } \\
& \text { the east-west direstion } \\
& \mathrm{T}_{\mathrm{NW}, \mathrm{SE}}=\text { antenna temperature of radiation polarized along } \\
& \text { the northwesi (southeast) direction } \\
& \mathrm{T}_{\mathrm{NE}, \mathrm{SW}} \text { - antenna temperature of radiation polarized along } \\
& \text { the northeast (southwest) direction }
\end{aligned}
$$

Stokes parameters are ideally suited for this experiment since the measured quantities differ from the Stokes parameters by a simple scale factor.

If the measuring instrument is initially aligned to measure $Q$, then physically rotating the instrument by $45^{\circ}$ gives $\mathrm{U}$ while in general a rotation by $90^{\circ}$ reverses the sign of $\mathrm{Q}$ and $\mathrm{U}$. This is shown schematically in Figure II.2. Usually instrumental effects are either constant with rotation or change sign under rotation by $180^{\circ}$. Rotating in $45^{\circ}$ increments for a full $360^{\circ}$ will therefore measure $Q$ and $U$ as well as the instrumental effects. This is a crucial aspect of the experiment, since we are attempting to measure polarization to a level which is typically onethousandth of the instrumental effect. 
FIgure II.2 - Stokes parameters $Q$ and $U$

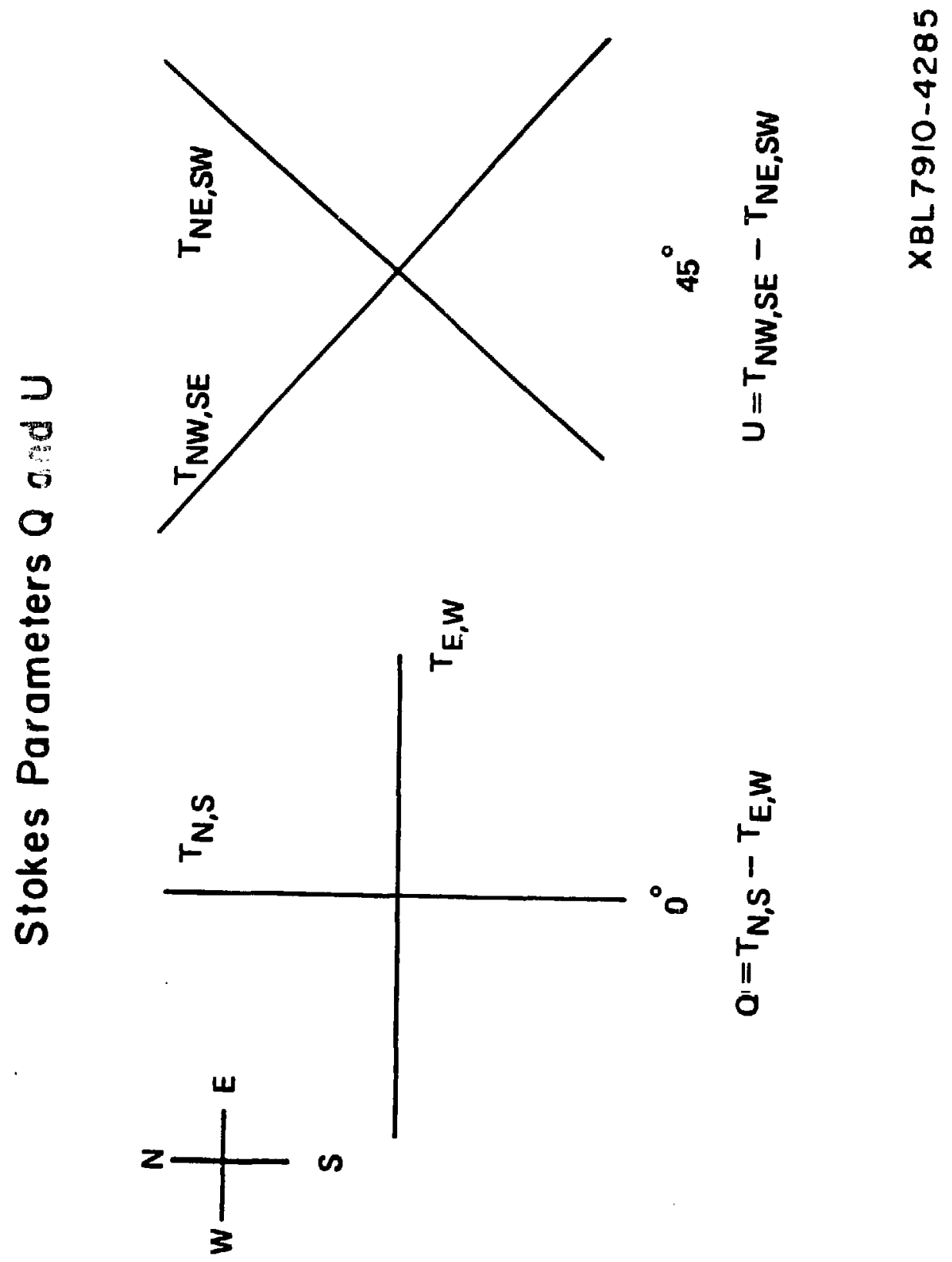




\section{Chapter III - Experimental Technique}

\section{III.1 Apparatus Description}

The apparatus is shown schematically in Figure III.1. The antenna axis can also be tilted relative to vertica!, in order to obtain various declination runs from one location. The ground shield aids in rejecting power from nearby objects, thermal and otherwise. A stepping motor rotates the radiometer ahout its axis on the bearing shown in eight $45^{\circ}$ increments to allow both Stokes parameters $\mathbf{Q}$ and $\mathbf{U}$ to be measured and to providr a basic summetry in order to cancel instrumental effects. A rain shield of 0.5 mil polyvinylidene (Saran Wrap) provides protection from rain and dust. Polyvinylidene is superior to many materials tested, because of its low attenuation and reflection of $9.1 \mathrm{~mm}$ radiation.

The radiometer is encased in a metal can which provides RF shielding. The radiometer is electrically insulated Irom the can, thus decoupling any possible grounding effects. The lockin amplifier uses an "ideal" integrator and a narrow band amplifier, $Q=10$, with a center frequency of $100 \mathrm{~Hz}$ and responds only to signals synchronous with the switching of the Faraday Rotation Switch. The output of the lockin is then digitized and recorded on a remote Kennedy 1600 incremental, 7-track tape recorder. Because the distance from radiometer to tape is typically 100 feet or more, a shielded twisted-pair line driver-receiver system is used to transmit and receive the data. This has the added virtue of eliminating any ground loops between the tape recorder and radiometer.

\section{III.2 Microwave Radiometer}

The radiometer is shown schematically in Figure III.2. It is a superheterodyne microwave receiver operating at $9.1 \mathrm{~mm}$ wavelength which can be rapidly switched between two polarization states. The output is proportional to the power difference in these two polarization states. As with all receivers, it has a sensitivity which is limited by its intrinsic noise. In our case this 
FIgure III.I - Polarimeter Schemat1c

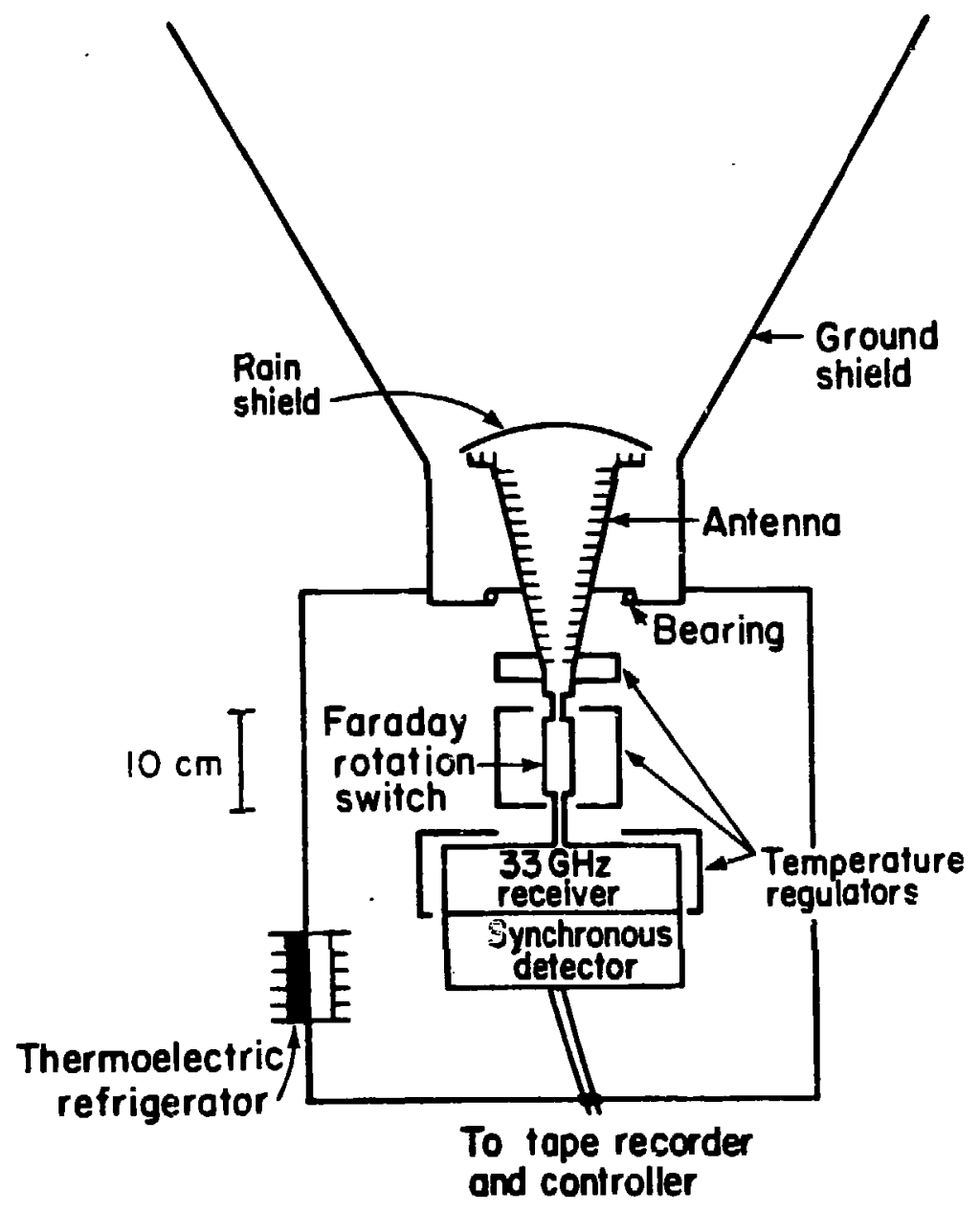

XEL 7910-4283A 


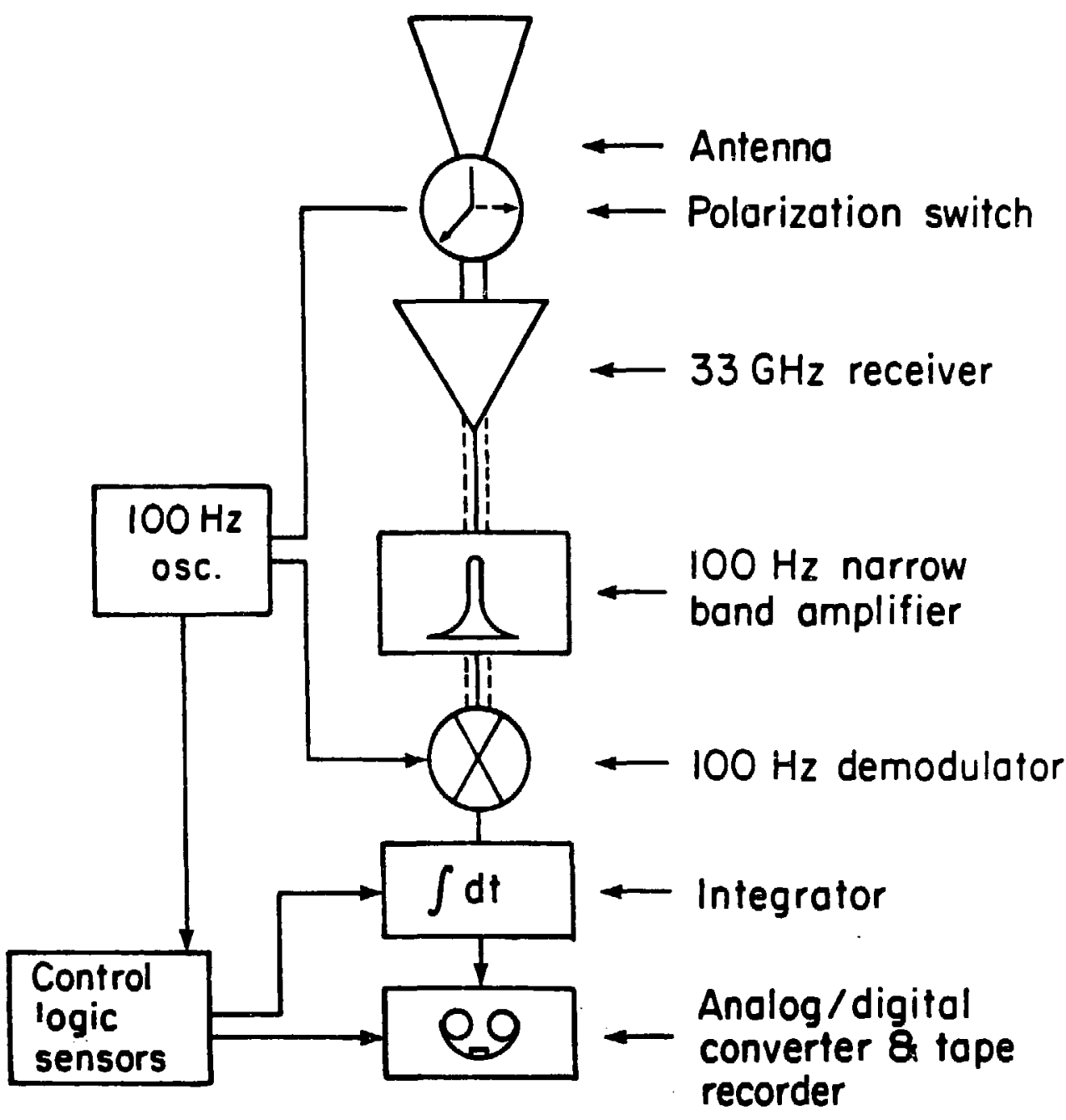

XBL $776-1338 A$ 
limit is specified by the minimum detectable temperature difference $\Delta T$ between the two polarization states. For a square-wave switched, narrow band detected radiometer, such as ours, $\Delta T=\frac{2.2 T_{\mathrm{sys}}}{\sqrt{B_{T}}}$ where $T_{\mathrm{sys}}$ is the system noise temperature (characteristic of system performance), $B$ is the IF bandwidth, and $\tau$ is the measurement time (Kraus, 1966). For our system, the system noise temperature is typically $T_{\text {sys }}=520^{\circ} \mathrm{K}$ and the IF bandwidth is $B=500 \mathrm{MHz}$, so $\Delta T=52 \mathrm{~m}^{\circ} \mathrm{K} / \mathrm{sec}^{1 / 2}$. Thus, by integrating (measuring) for a sufficient period of time, the desired sensitivity can be obtained. For example, integrating for one year allows us to reach a theoretical sensitivity of $0.01 \mathrm{~m}^{\circ} \mathrm{K}$.

\section{III.3 Thermal Regulation}

Thermal regulation is crucial, as various components are sensitive to temperature variations, particularly the Faraday Rotation Switch. The detailed requirements are discussed in the section on systematic error analysis (Appendix C). As shown in Figure III.I we thermally regulate three sections: the lower portion (throat) of the antenna, the Faraday Rotation Switch, and the microwave receiver. In addition, the lockin amplifier is temperature stabilized through attachment to the regulated block of the receiver.

Regulation is achieved by a combination of active and passive thermal elements. The three regulated areas have independent linear proportional heaters with feedback from a thermistor at the critical point. Thermal mass assures that heat is evenly distributed with a long time constant. By these two means, thermal regulation is achieved to $\pm 0.2^{\circ} \mathrm{C}$, except during times of excessive heat input, such as when the sun shines on the horn. Sufficient thermal mass (aluminum) assures that the time-rate. of change of the temperature is less than $0.3^{\circ} \mathrm{C}$ per hour. A thermoelectric refrigerator was installed for the southern hemisphere measurements because of the warm climate and was subsequently utilized in Berkeley. 


\section{III.4 Calibration}

Calibration is periodically performed using a polarized blackbody source at ambient temperature. The calibrator is shown in Figure III.3. Theoretical calculations (Chu, Gans \& Legg, 1975) and our radiometric measurements show that the calibrator is nearly ideal in that the polarized signal is equal to the difference in temperature between the reference blackbody (eccosorb) and sky. In practice we measure the eccosorb temperature each time, but only periodically measure the sky temperature. This simple procedure allows us to make an absolute calibration, good to better than $4 \%$ which is sufficient for our purposes. Additional calibration using the same receiver but with a Dicke switch in place of the Faraday Rotation Switch, agrees with the above method within $5 \%$.

The calibrator wire grid is made by photo-etching copper plated 2 mil Kapton. The wires are spaced $\mathbf{0 . 6 4} \mathrm{mm}$ on center. This dimension is not critical as long as it is small compared to the wavelength of $9.1 \mathrm{~mm}$. The grid is canted at a $45^{\circ}$ angle. Radiation whose electric field (polarization) is along the wire direction will be reflected, whereas radiation polarized perpendicular will be transmitted. This is precisely analogous to the optical case of a polaroid sheet, where the conduciive wires are provided by iodine ions on a stretched polymer grid (Shurcliff \& Ballard, 1962).

The calibration signal seen by the polarimeter is then a partially polarized signal, the magnitude of the polarized part being the difference in temperature between the eccosorb (ambient temperature blackbody source) and the sky (atmosphere plus background radiation). Independent measurements of the atmospheric contribution give $T_{1} \sim 12 \pm 1 \%$ for typical clear day. The presence of variable amounts of water vapor changes this by several degrees Kelvin. Adding the contribution of the cosmic background radiation of $2 \mathrm{~K}$ yields an atmospheric contribution of $T_{1}-14 \pm{ }^{\circ} \mathrm{K}$ where the skewed error is due to the variability of water vapor in the atmosphere.

Independent radiometric measurements at $33 \mathrm{GHz}$ give an insertion loss through the grid 
of $1.5 \pm 0.1 \%$ for the transmission mode and reflection of $0.99 \pm 0.01$ in the reflection mode. The eccosorb temperature is measured for each calibration with an error of less than $1 \%$. The total polarized signal is then $T_{\text {col }}=T_{\text {err }}-14 \%$ with an error of less than $4 \%$. 


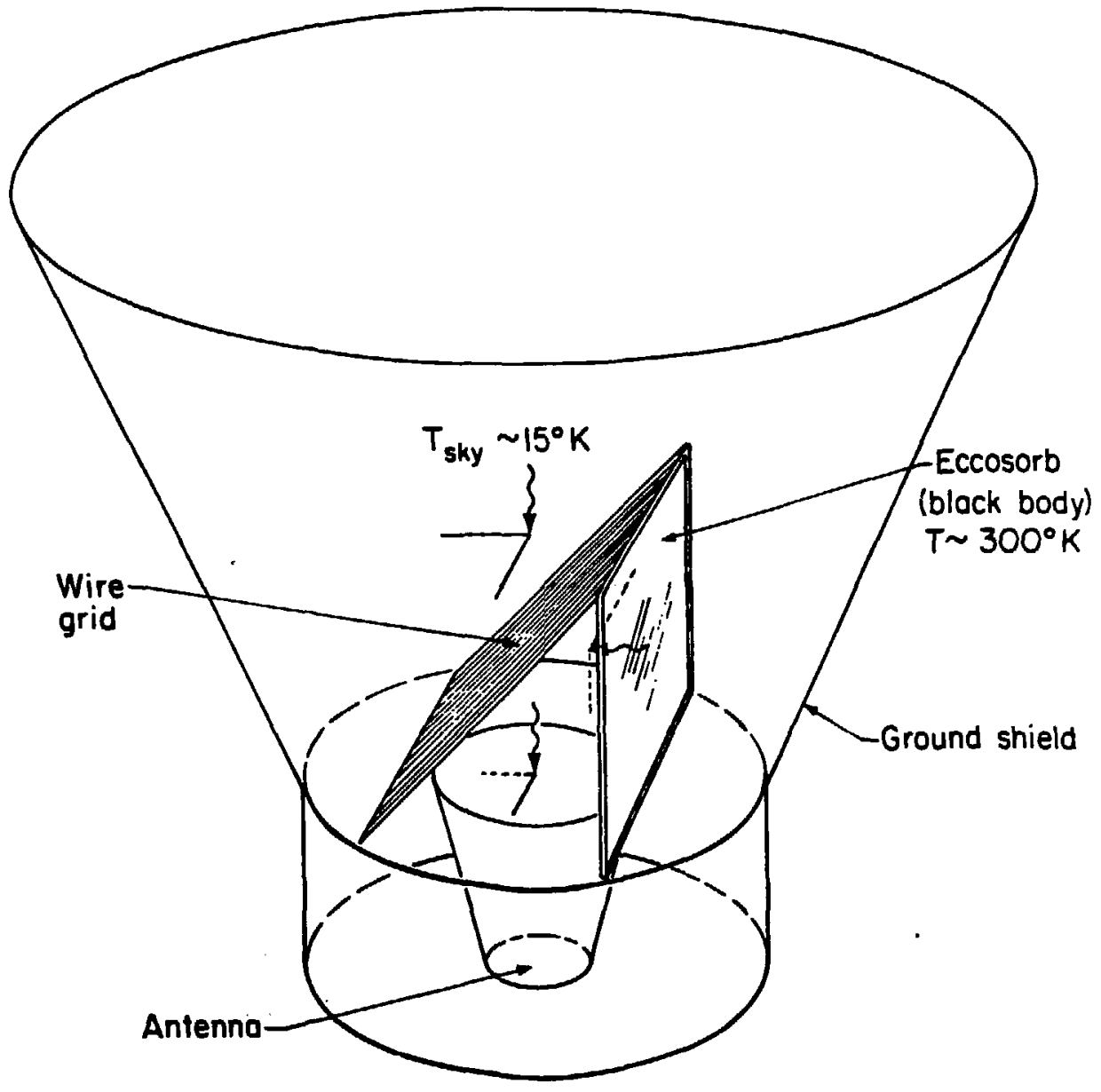

Calibration

XBL $601-140$

FIgure III.3 - Calibration 


\section{Chapter IV - Data Acquisition}

\section{IV.1 Data Recording}

The radiometer signal $\pm Q$ or $\pm U$ is integrated for 100 seconds after which it is digitized with 12 bit resolution and recorded. The radiometer is then rotated by $45^{\circ}$, and the process is repeated until a $315^{\circ}$ rotation has been achieved, after which the instrument rotates back to its $0^{\circ}$ initial position and the cycle repeats. The system is automated and runs unattended except for cleaning and occasional repair. The 7-track Kennedy 1600 tape recorder used has a readafter-write error checking ability, which is used to flag any improperly recorded data. A list of the recorded information is given in Table IV.1.

A typical tape contains about two weeks of data before being analyzed. After analysis this data is added to a lihrary tape containing all previous data. Time is recorded from a crystal controlled clock for later binning of data and correlation of time related events. The basic record structure consists of eight 40 bit words corresponding to a full rotation cycle. Each word corresponds to a rotation position and consists of the signal, time, rotation position, and various housekeeping signals. Each record is self contained ir that all information necessary to calculate the Stokes parameters is present. Data taken during jeriods of rain or dew is deleted. Humidity is recorded allowing an additional check of contaminated data.

\section{IV.2 Data Taking}

The northem declination data was taken from the Lawrence Berkeley Laboratory, Lat. = $38^{\circ} \mathrm{N}$. Heavy fog was apparently only a problem when it condensed on the rain shield. During periods of rain the equipment was either removed or covered. The ground shield was cleaned every week with alcohol and the rain shield was replaced at the same time.

Southem declination data was taken from the Naval Air Base at the Jorge Chavez airport 


\section{INFORMATION RECORDED ON TAPE}

\section{Analog (12 bit resolution)}

1 Data (Perfect Integrator)

2 Data (R C Integrator)

3 Polarization Switch - ferrite temperature

4 Polarization Switch - outer case temperature

5 Mixer $-I F$ temperature

6 Ambient temperature

7 Horn heater current

8 Polarization Switch heater current

9 Mixer - I F heater current

10 Ambient Humidity

Digital

1 Julian Day

2 Universal Time: Hours, Minutes, Seconds

3 Sense switch setting - for tagging data

4 Antenna rotation position

5 Bit recording error - tape recorder has read after write comparator. 
in Lima, Peru, Lat. $=12^{\circ} \mathrm{S}$. This was done simultaneously with our U-2 operations in Lima to measure the intensity anisotropy. Heat, dust, power failures, and logistics made the southern data taking less than optimal, but useful data was obtained nevertheless. In both hemispheres the instrument was aligned along the north-south direction such that $Q$ and $U$ were properly defined. Tilting of the instrument is done along the north-south direction so that as the earth sweeps the antenna beam along a constant declination the proper orientation of $Q$ and $U$ is maintained for each declination. During a typical run the instrument was pointed towards a fixed Jeclination for two weeks with a calibration at the beginning and the end of the run. Multiple runs were taken at most declinations. Figure IV.l shows the sky coverage obtained from both the northern and southern hemisphere. In tolal, eleven declinations were surveyed ranging from $-37^{\circ} 10+63^{\circ}$ declination. 


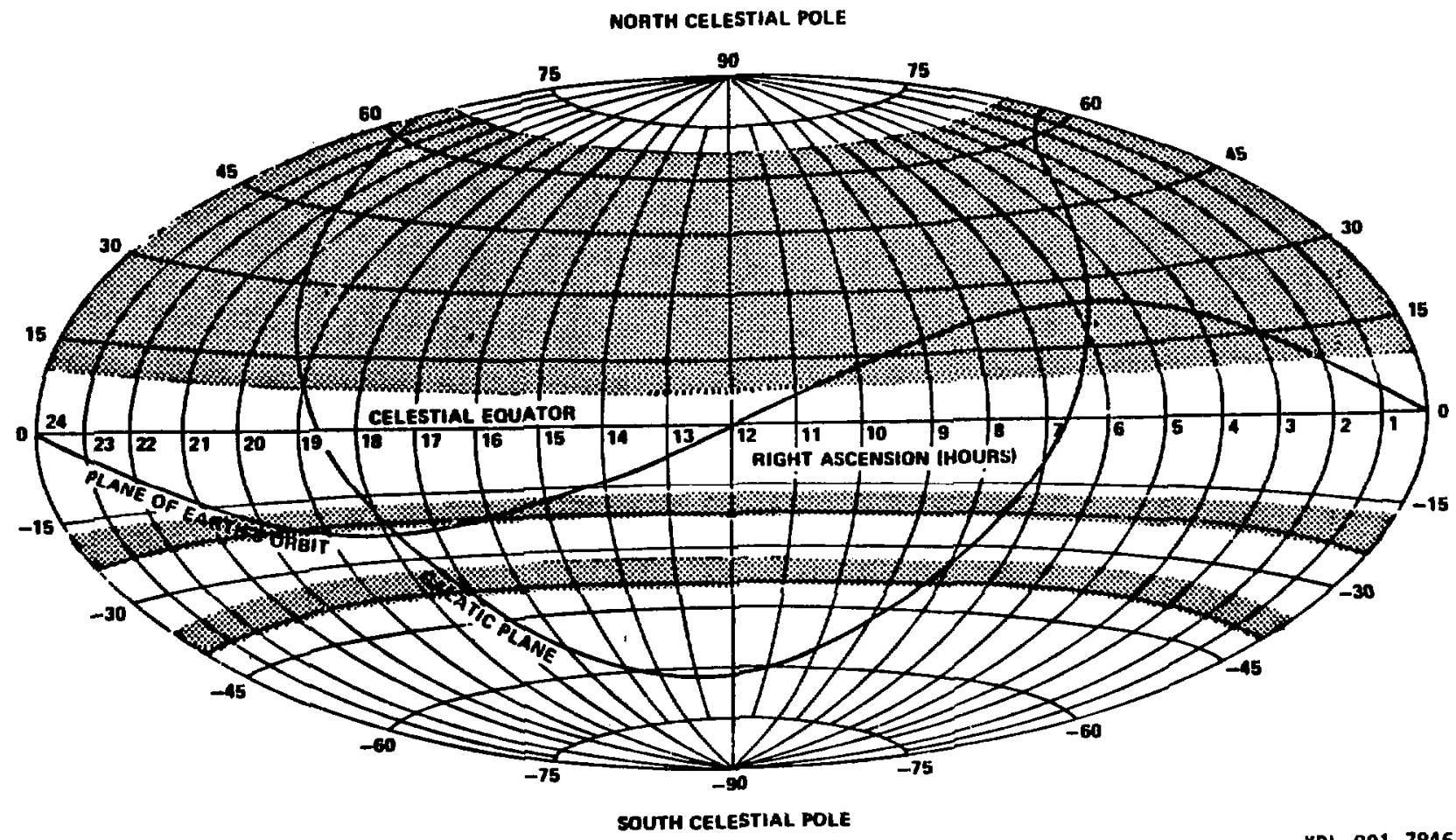




\section{Chapter V - Data Reduction and Analysis}

\section{V.1 Data Reduction}

To eliminate the instrumental ofiset (average DC output) and to obtain both components of linear polarization, the instrument is rotated in $45^{\circ}$ increments about the horn axis. If the offset were independent of rotation, then three consecutive positions would suffice to determine Q. U, and the offset, since this is equivalent to three equations in three unknowns. This is possible when the instrument axis is vertical, so as to minimize gravitational torques on the instrument. When the instrument axis is not vertical, gravitational forces cause the instrument to wobble slightly as it rotates. A full rotation in $45^{\circ}$ steps allows us to remove this wobble because of its mechanical nature. The "wobble correction" is discussed in appendix A.

A basic rotation cycle produces eight values $S_{1}, \ldots . S_{8}$, corresponding to rotation positions $0^{\circ}, 45^{\circ}, \ldots, 315^{\circ}$. The offset is constant with rotation angle lexcept for the wobble which changes sign under rotation by $180^{\circ}$ ), while any signal indicative of a true polarization would reverse sign upon rotation of the instrument by $90^{\circ} . Q$ and $U$ can thus be calculated as follows:

$$
\begin{aligned}
& Q=\left(S_{1}-S_{3}+S_{5}-S_{7}\right) / 4 \\
& U=\left(S_{2}-S_{4}+S_{6}-S_{5}\right) / 4
\end{aligned}
$$

The offset is calculated as the average of $S_{1}, \ldots, S_{1}$.

Siderial time is calculated for each value of $Q$ and $U$ from the recorded universal time. $A$ least-squares fit is done to Fourier components with periods of DC (constant) 24, 12, 8, 6, and 4.8 hours for $Q$ and $U$ at each declination observed. $Q$ and $U$ are binned in hourly siderial bins and time plots are made. Global fits are made by doing a least-square fit to the hourly bins of each declination, using a series of spherical harmonics as fitting functions.

A diagnostic program is run on the data to test their statistical properties. Table V.I lists the statistical tests performed. 


\begin{tabular}{|c|c|}
\hline & $\begin{array}{c}\text { Table V.1 } \\
\text { Statistical Tests of Data Performed }\end{array}$ \\
\hline Fourier Transform & Test for Spurious Periodic Signals \\
\hline Run Test & Test for Random Nature of Data Above and Below Mean \\
\hline Gaussian Statistics & $\begin{array}{l}\text { Check for Gaussian Nature of Data and look for } \\
\text { Non-statistical Behavior in Tails of Distribution }\end{array}$ \\
\hline Integration Test & $\begin{array}{l}\text { Check for low level systematic errors } \\
\text { by plotting RMS luctuations of } \\
\text { binned data against number of data } \\
\text { points in each bin. The fluctuations } \\
\text { should average down inversely as } \\
\text { the square root of the number of } \\
\text { data points in each bin. }\end{array}$ \\
\hline
\end{tabular}

Recall that the minimum detectable signal is inversely proportional to the square root of the integration time. This is of particular importance as it tells us whether or not the data "integrates down" properly. Such a test is done on the data in the diagne" "rogram and a sample of this is shown in Figure V.I. The $\frac{1}{\sqrt{N}}$ line is drawn in for comparison.

\section{V.2 Analysis Algorithm}

The reduction of data from the tape to the actual limits on cosmological models involves a number of diverse steps. Figure V.2 shows the general algorithm we use in the analysis. A typical analysis of a tape proceeds as follows. A calibration is performed just before removal of the tape. The tape is then checked for bad records on an IBM 1401 and if any are found they are deleted. An initial run is performed to produce time plots and fits to $Q$ and $U$. This is inspected and any obviously bad data are deleted (see next section); the data are rerun to produce fits for the particular declination. Cards are automatically punched of the hourly siderial averages of $Q$ and $U$. The diagnostic program is then run for statistical testing of the data. $A$ permanent copy of the tape is made. Global analysis proceeds using the punched hourly averages of $Q$ and $U$ at each declination. Calibration constants for each run are folded into $Q$ and $U$ at each declination to convert from digital units to millidegrees Kelvin. 


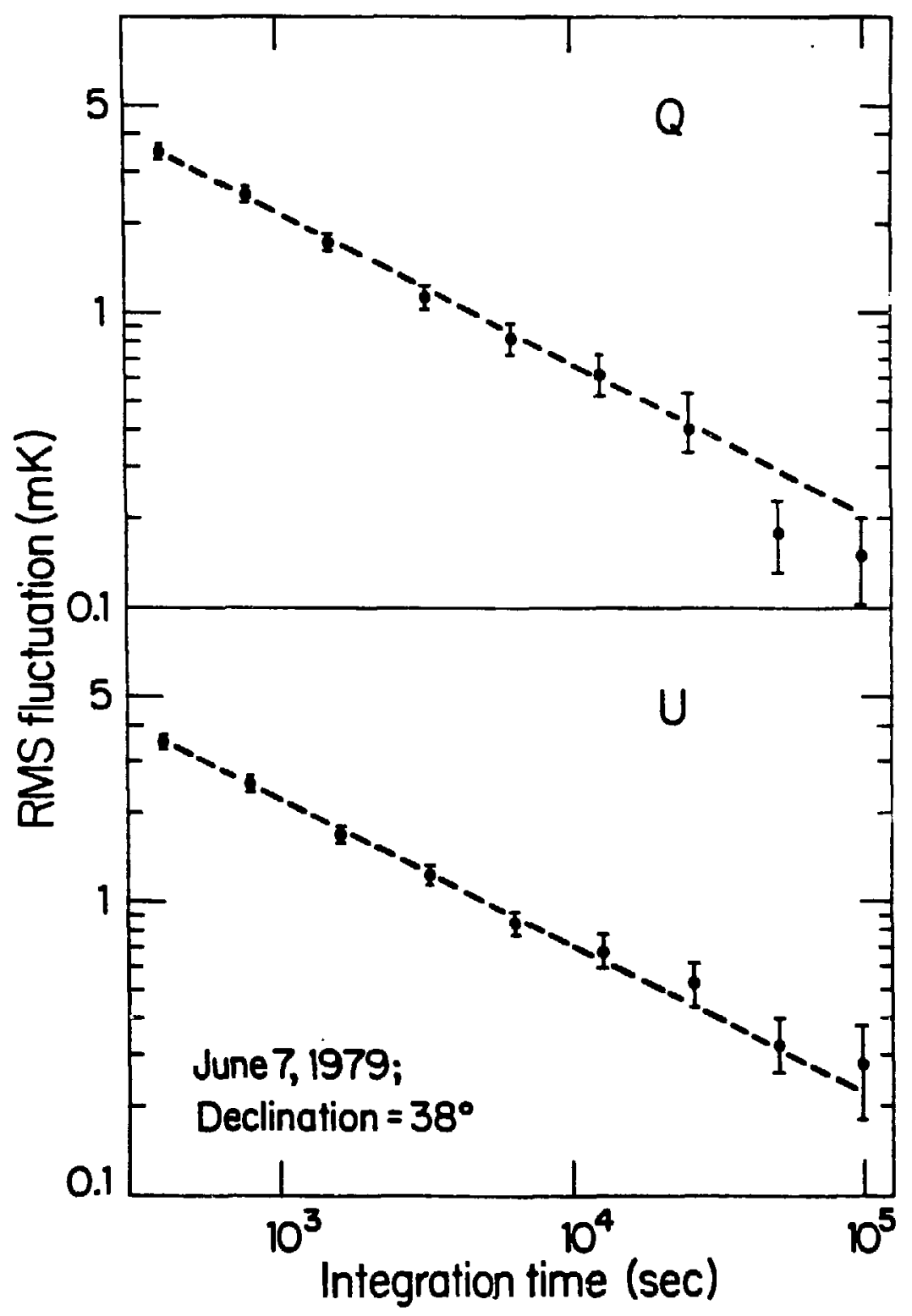

XBL $802-237$

Figure V.I - RMS Fluctuation versus Integration Time 


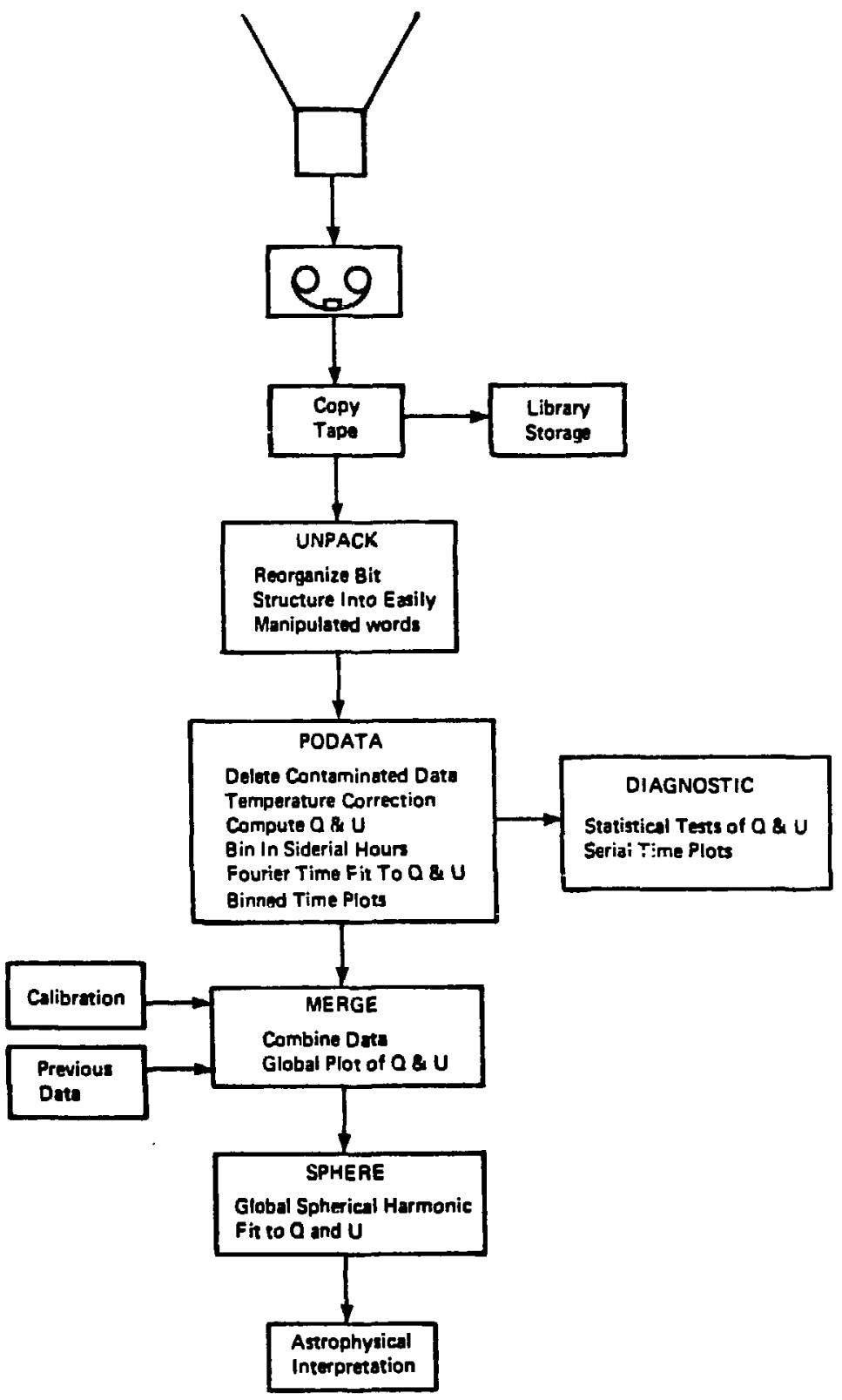

XBL799-3003

FIgure V.2 - Data Analysis Algorithm 


\section{V.3 Data Deletion}

Deleted or edited data falls into two categories: data which can be eliminated because of known causes (sun overhead, rain, cleaning ground shield, and data which have obvious nonstatistical behavior but whose cause is known. The latter category is somewhat more difficult to quantify in terms of a rejection threshold.

Our philosophy is to use all data which are not "obviously" bad, so as not to bias the results. In this experiment there are large quantities of data, so it is crucial to constantly check for consistency lest a real signal be randomized. 


\section{Chapter VI - Data and Fits}

\section{VI.1 Data}

Table VI.1 gives a list of the data and errors for each declination surveyed. These data have been corrected for the temperature dependence of the Faraday Rotation switch, and for the instrument wobble in runs where the apparatus is not pointed vertically. The northern hemisphere data consist of several runs at each declination which are merged to form the data in Table VI.1. Figure VI.1 shows the data in graphical form for each declination. Because of the restriction imposed by contamination from the sun and the limited time in the southern hemisphere, the errors are not equal for each declination. The errors given are actual errors, not just " statistical errors based on receiver noise only ".

\section{VI.2 Polarization Limits at Each Declination}

For each declination surveyed, a least-squares fit to Fourier elements of various periods is performed. The functions, fitted values and errors are given in Table VI.2.

\section{VI.3 Spherical Hammonic Fits}

A least-squares fit to various spherical harmonics is made using the binned hourly data given in Table VI.1. The fitting functions, amplitudes and errors are given in Table VI.3. These are independent fits to the average, dipole, quadrupole and octupole spherical harmonics. As can be seen from Table VI.3, none of the fits is very significant.

A fit to the null hypothesis of no polarization yields a chi-square of 279 with 264 degrees of freedom and a corresponding confidence level of $25 \%$ for $Q$, and a chi-square of 265 with 264 degrees of freedom and a confidence level of $47 \%$ for $U$. In addition, the model of Rees 


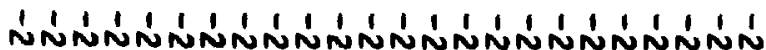

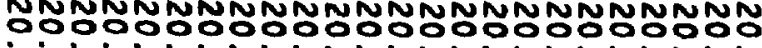

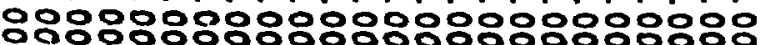

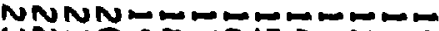

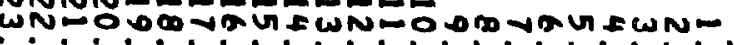

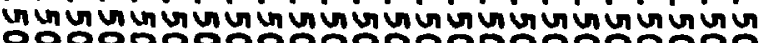

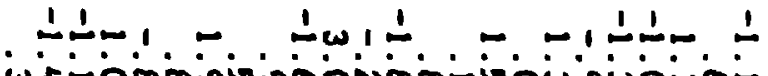

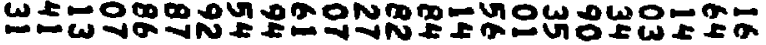

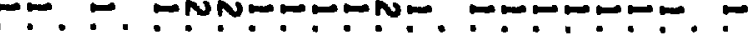

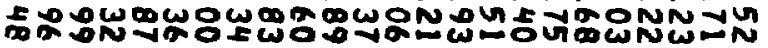

—! . N.

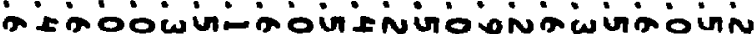

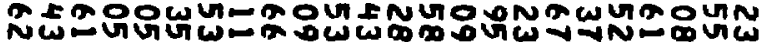

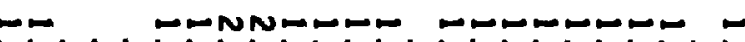
WPBO

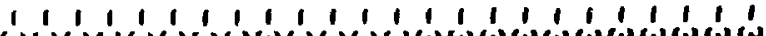

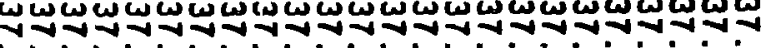

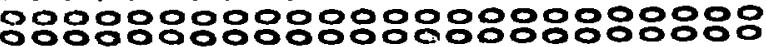

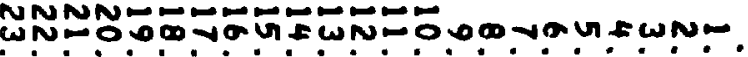

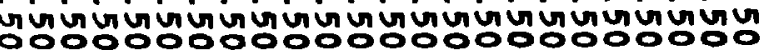

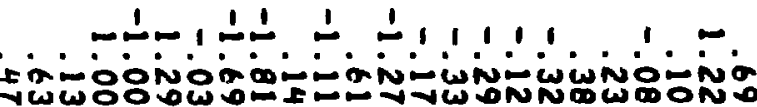

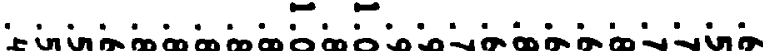

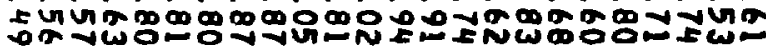

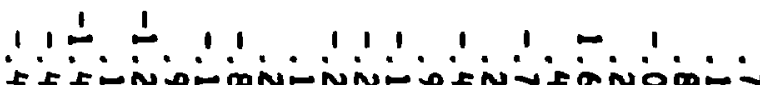

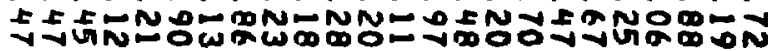

$\boldsymbol{D}$

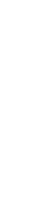

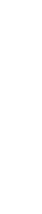

.

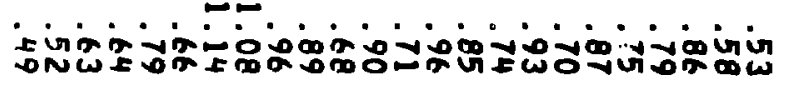




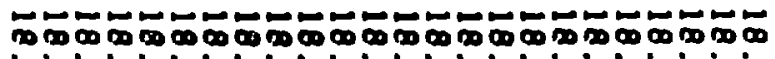

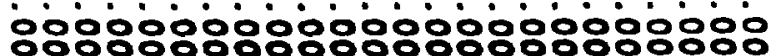

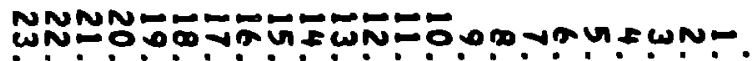

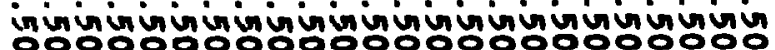

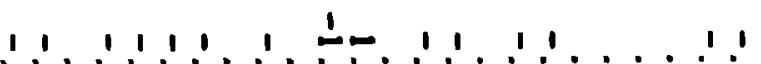

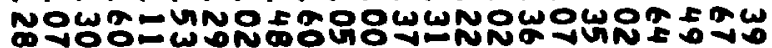

stson

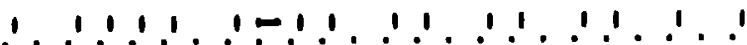

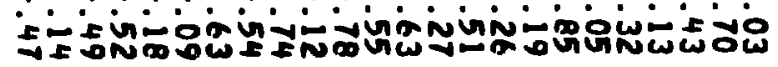

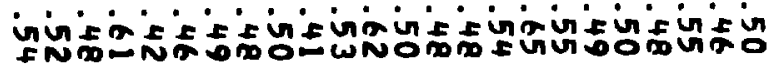

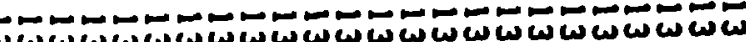
\%

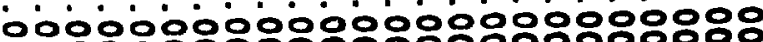

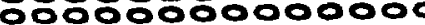

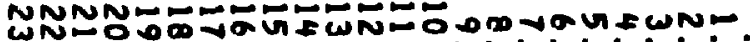

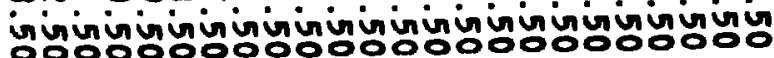

$\mathbf{D}$

is

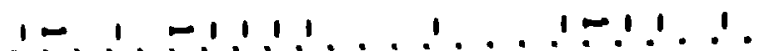
-

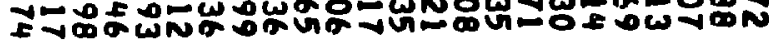

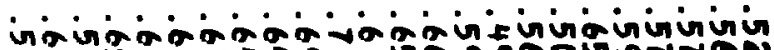

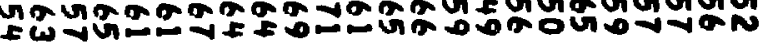

㽚

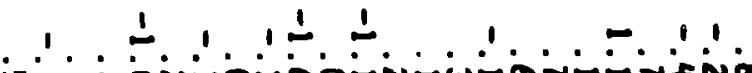

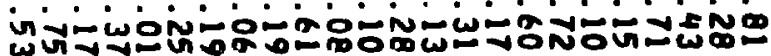

$r$

m ininging 
NNNNNNNNNNNNNMNNNNNNNNNN

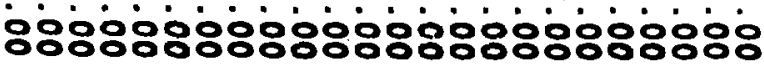

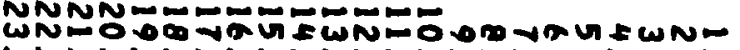

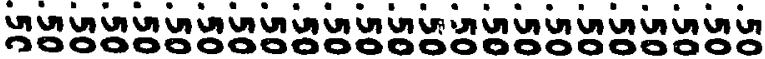

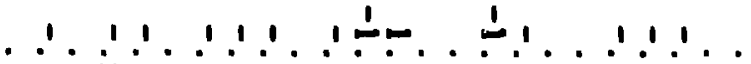
wW

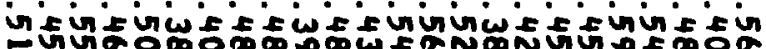

1

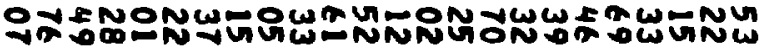

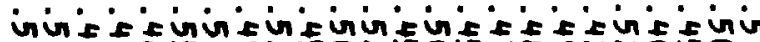
MUE⿱

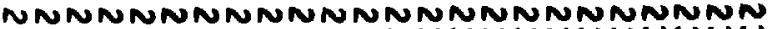
WWWWWWWWW WWWWWWWWWWWWW 808880880808080808808880

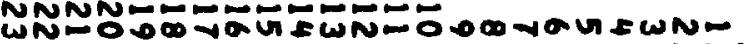

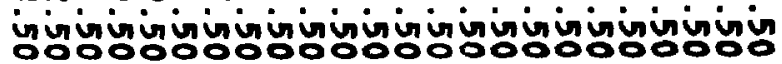

$\boldsymbol{\nabla}$

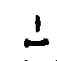

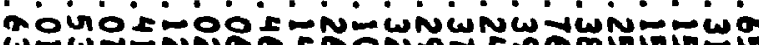

$\sigma$

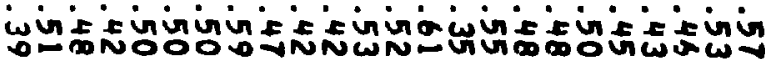




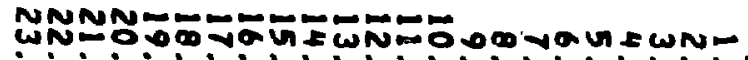
invinivinivininivinivinirinininin 000000000000000000060000

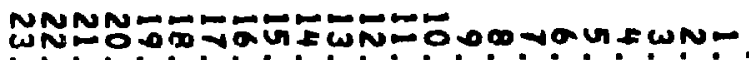

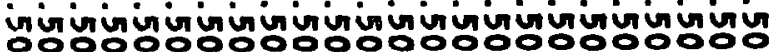

$\therefore 1.1-1111.1111111$ पूँ

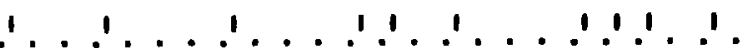

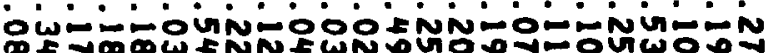

$\mathbf{D}$ 


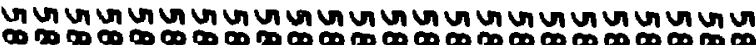

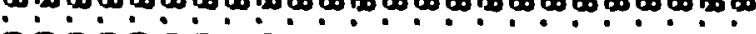

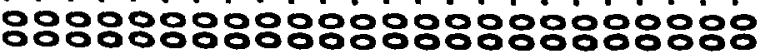

WNNSW

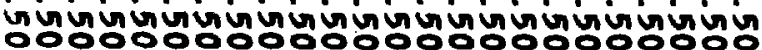

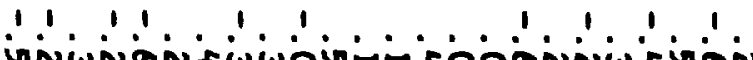

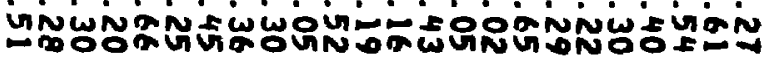

11 जท $\omega$ w

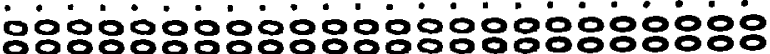

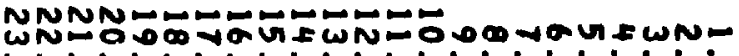

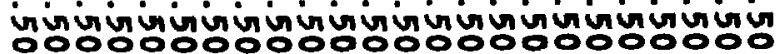

111

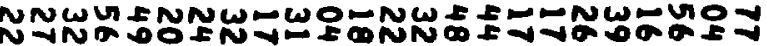

8

n

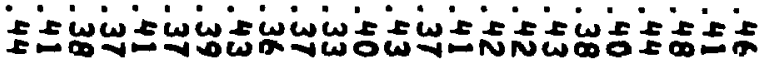

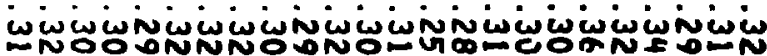

$\stackrel{\mathbf{m}}{\mathbf{3}}$

$\infty$

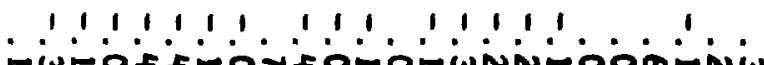

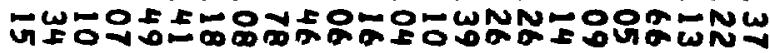

$\approx$

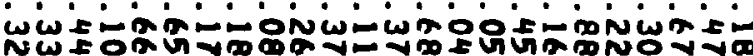

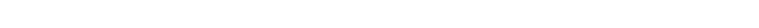

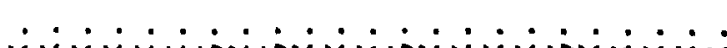
WWWWWWWNWNWWWNWWWWNWWWWW 


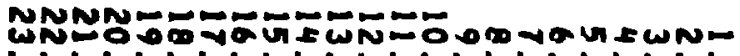
inirininirinininininininininin

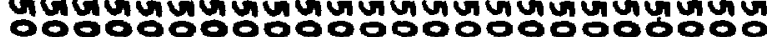

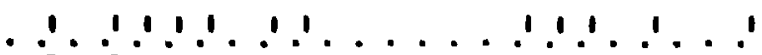

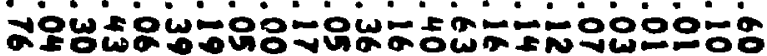

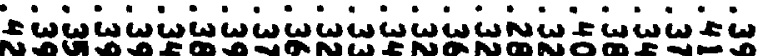

孚

$111111-1111111$

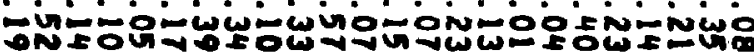

- sw 
Figure VI.1 - P1ot of Data

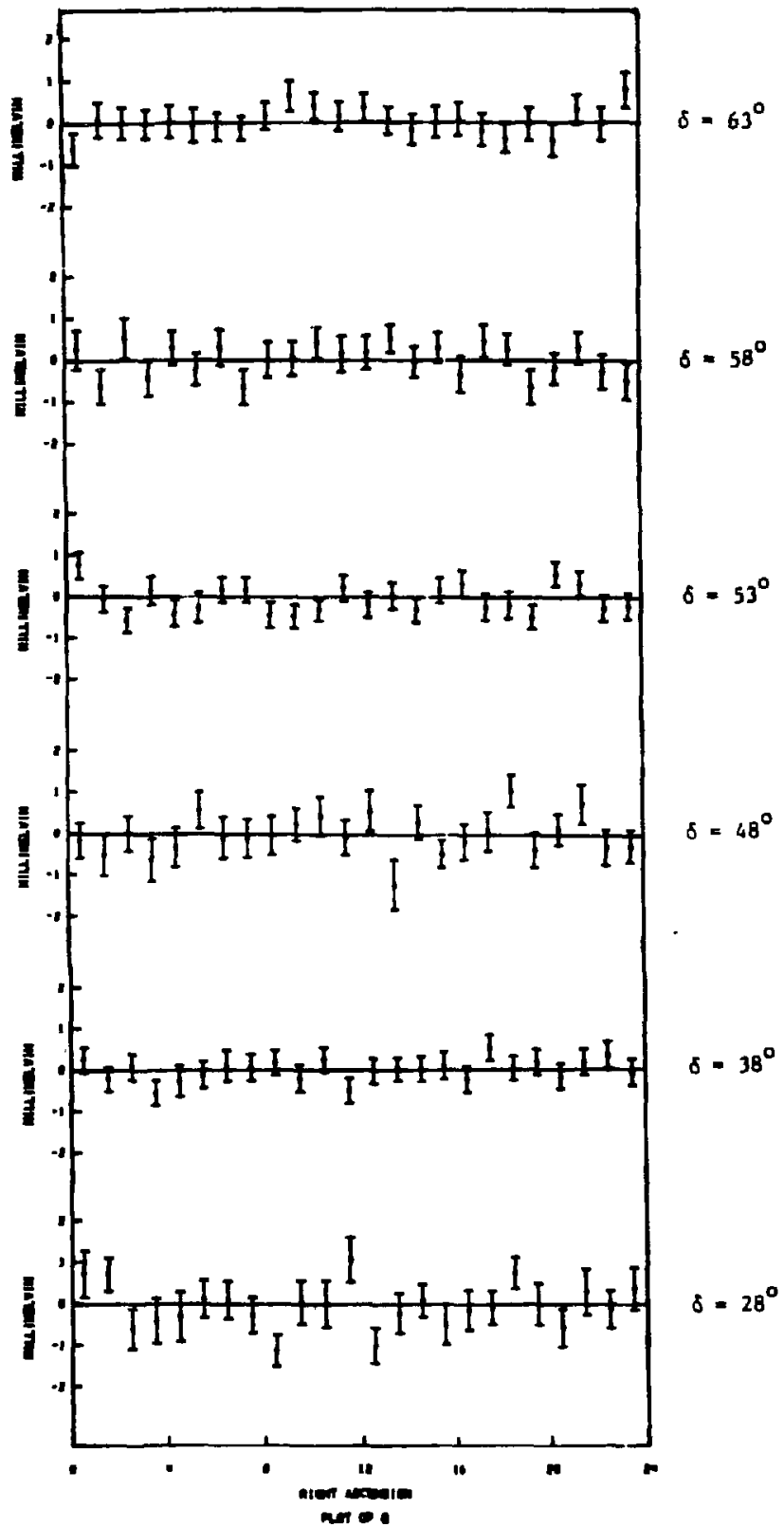

XBL 802-8409

Q 
Figure VI. 1

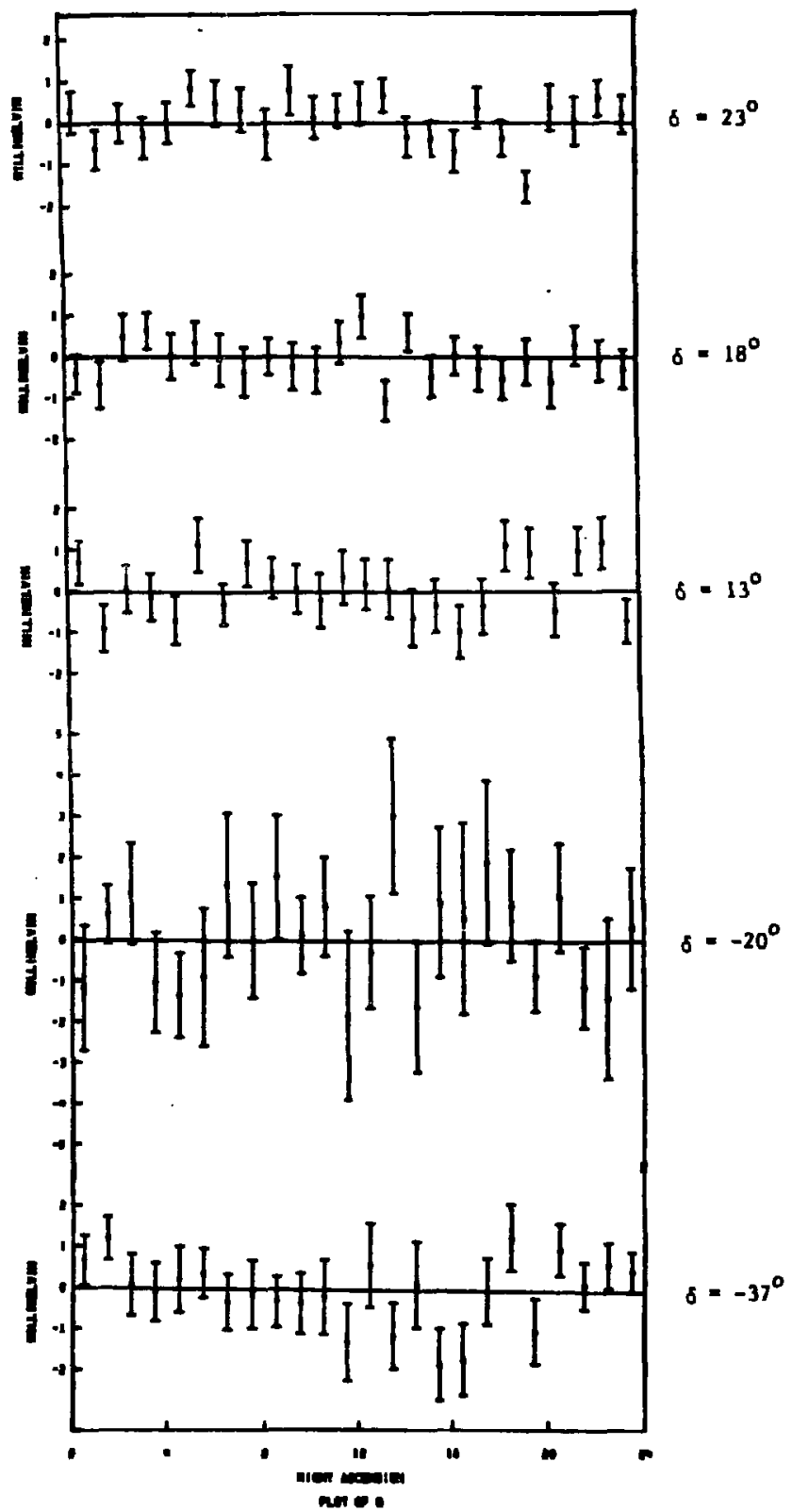

XBL $802-8412$ 
F1gure VI.1

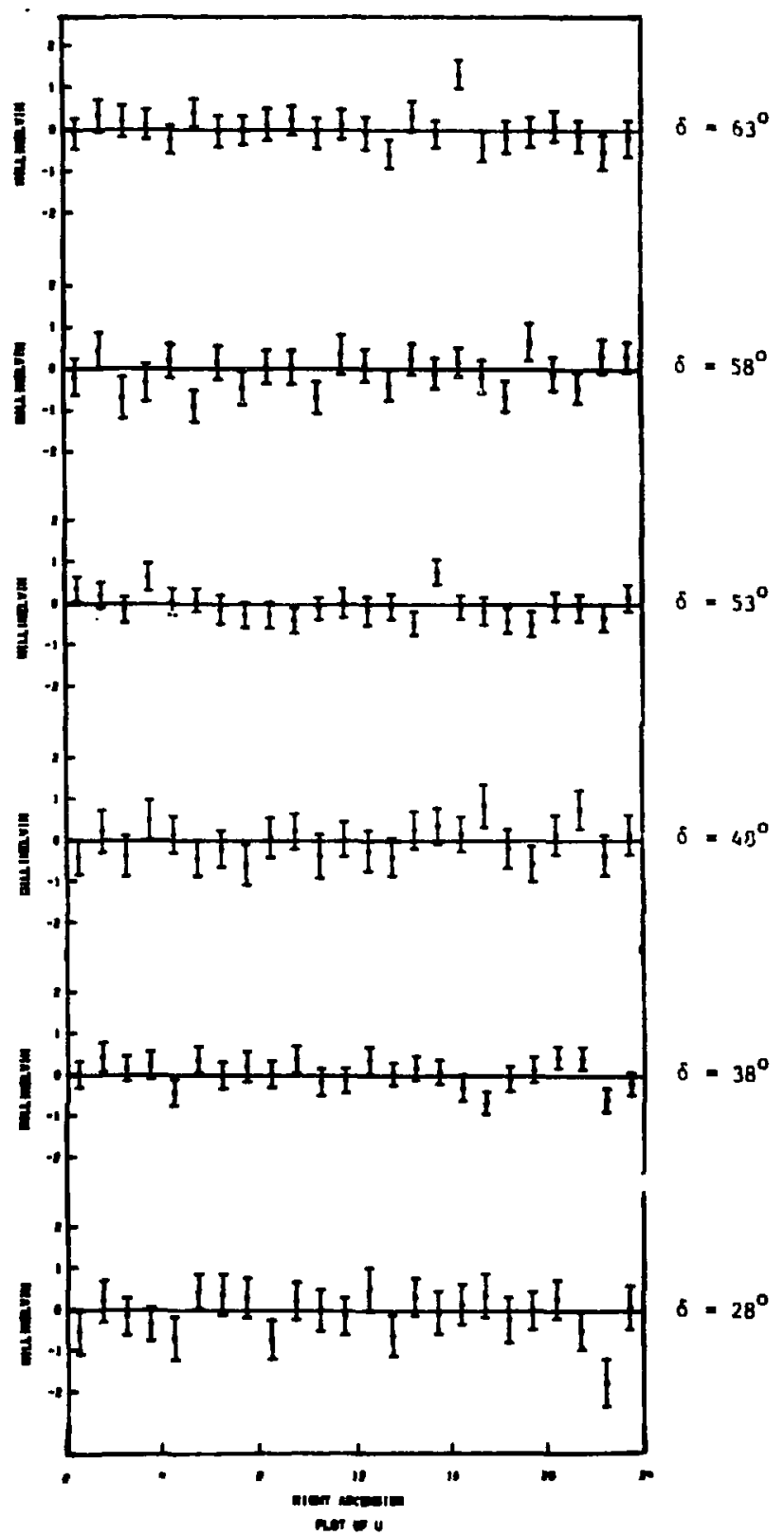

XBL 802-8411 
Figure VI. 1

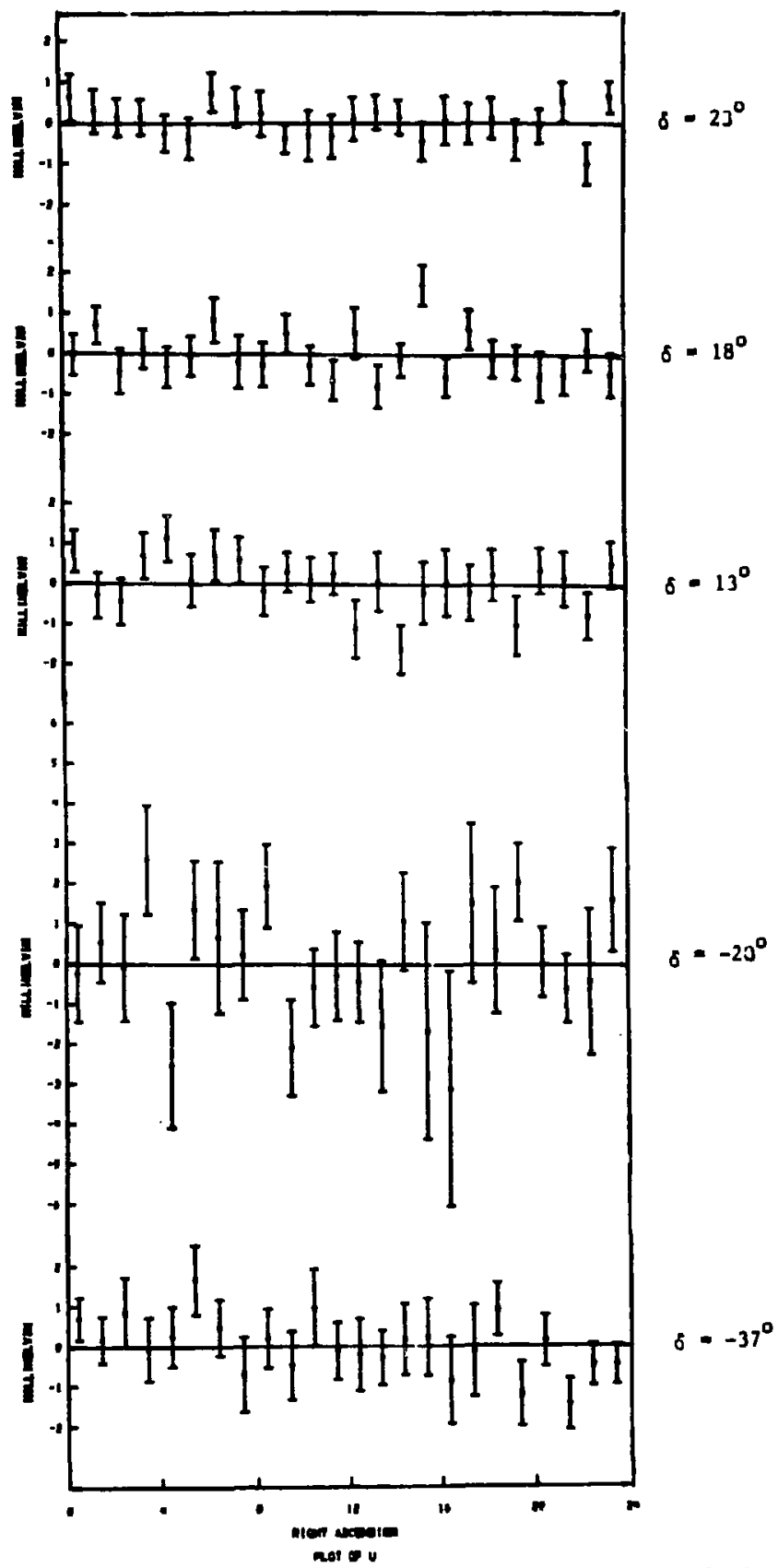

XBL $802-8410$ 


\begin{tabular}{|c|c|c|c|c|c|c|}
\hline 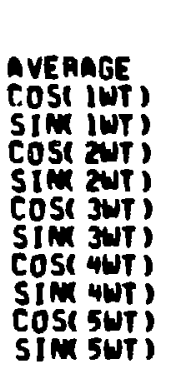 & $\begin{array}{r}0 \\
.11 \\
.68 \\
.14 \\
.27 \\
-.11 \\
-.03 \\
.06 \\
.21 \\
.20 \\
.10 \\
.03\end{array}$ & $\begin{array}{c}\text { DECLI } \\
\text { EAROA } \\
.14 \\
.20 \\
.21 \\
.20 \\
.20 \\
.21 \\
.20 \\
.20 \\
.21 \\
.21 \\
.20\end{array}$ & $\begin{array}{l}\text { Whion = } \\
\text { CHISO } \\
28.22 \\
16.67 \\
26.16 \\
28.73 \\
26.84 \\
28.60\end{array}$ & $\begin{array}{r}-31 \\
4 \\
-.01 \\
-.04 \\
.39 \\
-.10 \\
.39 \\
.01 \\
.30 \\
.27 \\
.17 \\
.29 \\
.20\end{array}$ & $\begin{array}{l}\text { EAROA } \\
.15 \\
.19 \\
.23 \\
.20 \\
.22 \\
.21 \\
.21 \\
.21 \\
.21 \\
.21 \\
.20\end{array}$ & $\begin{array}{l}\text { Ch1 S0 } \\
21.49 \\
18.44 \\
17.61 \\
19.30 \\
19.06 \\
18.10\end{array}$ \\
\hline 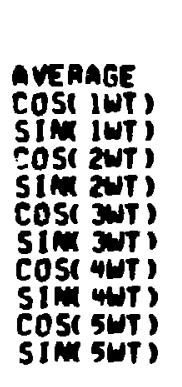 & $\begin{array}{r}9 \\
.01 \\
-.25 \\
.03 \\
.13 \\
.04 \\
.48 \\
.46 \\
-.03 \\
.43 \\
-.30 \\
.08\end{array}$ & $\begin{array}{c}\text { DECLI } \\
\text { EAROA } \\
.26 \\
.36 \\
.38 \\
.40 \\
.35 \\
.39 \\
.36 \\
.40 \\
.35 \\
.36 \\
.39\end{array}$ & $\begin{array}{c}\text { NTION = } \\
\text { CHISO } \\
16.73 \\
16.24 \\
16.59 \\
13.23 \\
15.18 \\
15.90\end{array}$ & $\begin{array}{r}-20 \\
.21 \\
.39 \\
.09 \\
-.53 \\
.05 \\
.08 \\
.09 \\
.06 \\
.66 \\
.28 \\
-.23\end{array}$ & $\begin{array}{c}\text { EAROA } \\
.25 \\
.34 \\
.39 \\
.37 \\
.34 \\
.35 \\
.36 \\
.35 \\
.36 \\
.35 \\
.36\end{array}$ & $\begin{array}{l}\text { CHISO } \\
24.92 \\
24.34 \\
23.58 \\
25.52 \\
22.21 \\
24.64\end{array}$ \\
\hline 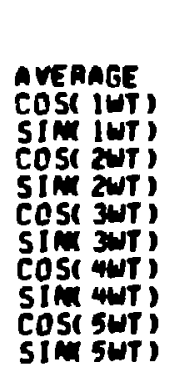 & $\begin{array}{r}.10 \\
.08 \\
-.01 \\
=.04 \\
=.43 \\
=.12 \\
-.09 \\
.09 \\
.15 \\
=.00 \\
-.10\end{array}$ & $\begin{array}{c}\text { DECLI } \\
\text { EAROA } \\
-12 \\
.17 \\
117 \\
117 \\
117 \\
117 \\
117 \\
117 \\
117 \\
.17 \\
117\end{array}$ & $\begin{array}{c}\text { Mrion }= \\
\text { CHISO } \\
29.62 \\
30.00 \\
23.00 \\
29.43 \\
29.11 \\
29.10\end{array}$ & $\begin{array}{l}13 \\
.0 \\
.08 \\
.20 \\
.43 \\
-.11 \\
-.11 \\
-.01 \\
.09 \\
.18 \\
-.20 \\
.08 \\
.05\end{array}$ & $\begin{array}{c}\text { EAPOA } \\
.12 \\
.17 \\
.18 \\
.18 \\
.17 \\
.18 \\
.17 \\
.17 \\
.18 \\
.17 \\
.18\end{array}$ & $\begin{array}{l}\text { CHISO } \\
26.14 \\
19.94 \\
25.47 \\
26.35 \\
24.15 \\
26.28\end{array}$ \\
\hline 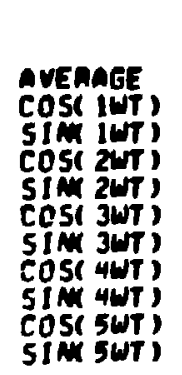 & $\begin{array}{l}=.04 \\
-.06 \\
.17 \\
.02 \\
.11 \\
=.22 \\
=.09 \\
=.09 \\
=.15 \\
=.17 \\
=.06\end{array}$ & $\begin{array}{c}\text { OECL } \\
\text { EAAOA } \\
.10 \\
.15 \\
.15 \\
.15 \\
.15 \\
.15 \\
.15 \\
.14 \\
.15 \\
.15 \\
.15\end{array}$ & $\begin{array}{l}\text { MTION } \\
\text { CHISg } \\
20.89 \\
19.69 \\
20.48 \\
10.35 \\
19.66 \\
19.51\end{array}$ & $\begin{array}{l}10 \\
-4 \\
-.00 \\
-.05 \\
.02 \\
-.12 \\
.16 \\
.28 \\
.07 \\
-.02 \\
.00\end{array}$ & $\begin{array}{c}\text { EAA9A } \\
.10 \\
.15 \\
.14 \\
.15 \\
.14 \\
.15 \\
.14 \\
.15 \\
.14 \\
.14 \\
.15\end{array}$ & $\begin{array}{l}\text { CHI } 50 \\
30.05 \\
29.93 \\
28.04 \\
26.36 \\
30.03 \\
28.78\end{array}$ \\
\hline
\end{tabular}

Table VI.2 - Ind1vidual Declination Fits 
$Z \cdot I \Lambda$ әTqEI

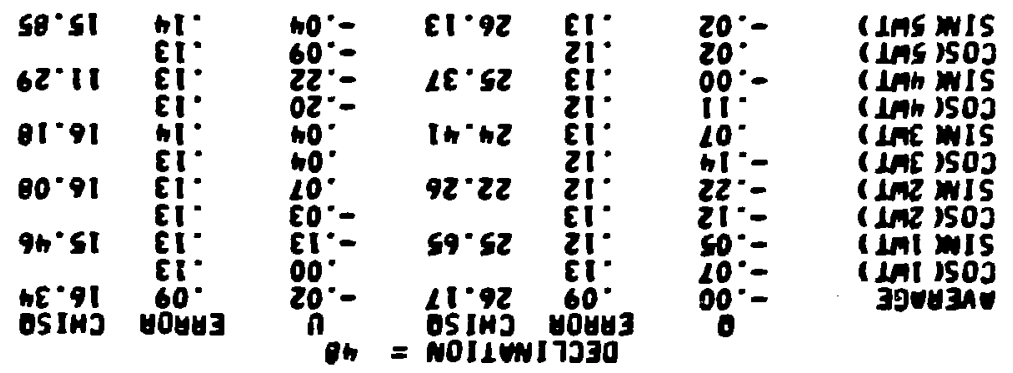

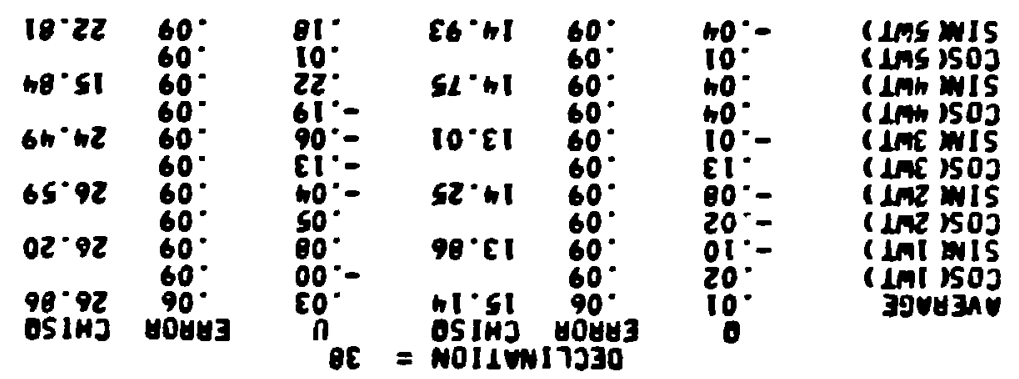

\begin{tabular}{|c|c|c|c|c|c|c|}
\hline $\begin{array}{l}86 \cdot 81 \\
26 \cdot 12 \\
16 \cdot c 2 \\
68 \cdot 12 \\
26 \cdot 12 \\
66 \cdot 22 \\
05145\end{array}$ & 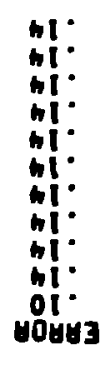 & 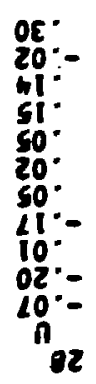 & 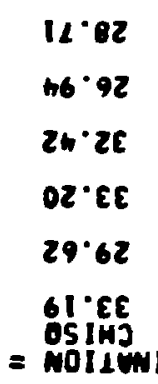 & 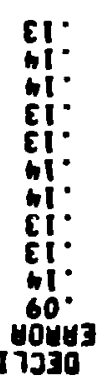 & 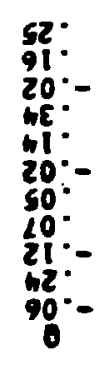 & 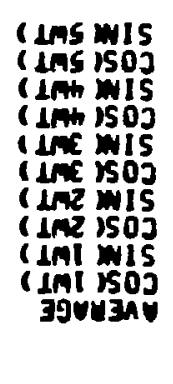 \\
\hline $\begin{array}{l}21 \cdot 21 \\
06 \cdot 21 \\
25 \cdot 91 \\
15 \cdot 11 \\
00 \cdot 91 \\
69^{\circ} 11 \\
051 \% 3\end{array}$ & 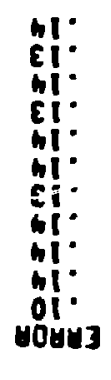 & 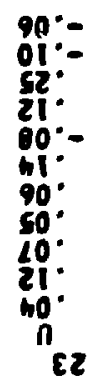 & 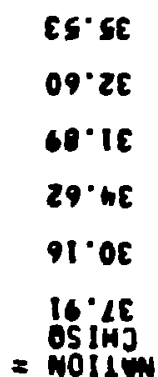 & $\begin{array}{l}n 1: \\
n 1: \\
81: \\
n 1: \\
n 1: \\
n 1: \\
n 1: \\
n 1: \\
01: \\
\text { noyns }\end{array}$ & 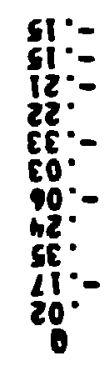 & 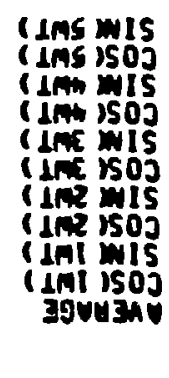 \\
\hline
\end{tabular}




\begin{tabular}{|c|c|c|c|c|c|c|}
\hline 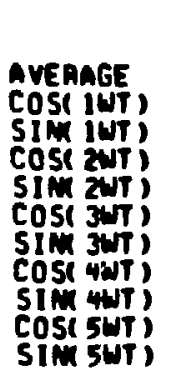 & $\begin{array}{r}-.07 \\
.09 \\
-.09 \\
.02 \\
.02 \\
.06 \\
-.13 \\
.06 \\
.94 \\
-.16\end{array}$ & $\begin{array}{c}\text { DECLI } \\
\text { EAROA } \\
.06 \\
.09 \\
.09 \\
.09 \\
.09 \\
.09 \\
.09 \\
.09 \\
.09 \\
.09 \\
.09\end{array}$ & $\begin{array}{l}\text { NATION = } \\
\text { CHISO } \\
30.54 \\
29.91 \\
31.89 \\
29.12 \\
31.33 \\
25.12\end{array}$ & $\begin{array}{r}53 \\
y \\
-.06 \\
.10 \\
.07 \\
.07 \\
.21 \\
.04 \\
-.01 \\
-.00\end{array}$ & $\begin{array}{c}\text { EAnOA } \\
.06 \\
.09 \\
.09 \\
.09 \\
.09 \\
.09 \\
.09 \\
.09 \\
.09 \\
.09 \\
.09\end{array}$ & $\begin{array}{l}\text { CHISO } \\
25.10 \\
23.91 \\
19.57 \\
25.70 \\
24.60 \\
23.96\end{array}$ \\
\hline 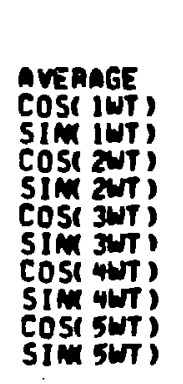 & $\begin{array}{l}2 \\
0 \\
5 \\
6 \\
9 \\
9 \\
1 \\
13\end{array}$ & $\begin{array}{c}\text { DECLI } \\
\text { EAAOA } \\
.08 \\
.12 \\
.12 \\
.12 \\
111 \\
.12 \\
.11 \\
.12 \\
.11 \\
.12 \\
.11\end{array}$ & $\begin{array}{c}\text { Mrion = } \\
\text { ChISO } \\
21.04 \\
10.01 \\
20.22 \\
20.60 \\
20.64 \\
20.94\end{array}$ & $\begin{array}{c}58 \\
u \\
-.08 \\
.05 \\
-.08 \\
.12 \\
.03 \\
10\end{array}$ & $\begin{array}{c}\text { Eดดดด } \\
.08 \\
.12 \\
.11 \\
.11 \\
.12 \\
.12 \\
.11 \\
.12 \\
.11 \\
.12 \\
.11\end{array}$ & $\begin{array}{l}\text { CHISO } \\
23.43 \\
23.79 \\
23.24 \\
23.69 \\
24.39 \\
24.37\end{array}$ \\
\hline 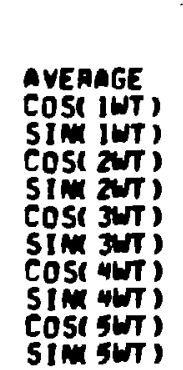 & 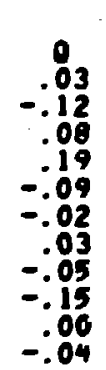 & $\begin{array}{c}\text { OECLI } \\
\text { EAROA } \\
.07 \\
.10 \\
.10 \\
.10 \\
.10 \\
.10 \\
.10 \\
.10 \\
.10 \\
10\end{array}$ & $\begin{array}{l}\text { MuTION = } \\
\text { CHISO } \\
16.00 \\
14.32 \\
12.23 \\
16.11 \\
13.75 \\
16.06\end{array}$ & $\begin{array}{r}63 \\
-.4 \\
.04 \\
-.05 \\
.02 \\
-.11 \\
.10 \\
.10 \\
.12 \\
-.08 \\
-.09\end{array}$ & $\begin{array}{c}\text { ERAOA } \\
-.07 \\
.11 \\
.10 \\
.10 \\
.10 \\
.10 \\
.10 \\
.10 \\
.10 \\
.10 \\
.10\end{array}$ & $\begin{array}{l}\text { CH150 } \\
26.51 \\
26.57 \\
24.83 \\
24.57 \\
25.56 \\
21.95\end{array}$ \\
\hline
\end{tabular}




\begin{tabular}{|c|c|c|c|}
\hline \multicolumn{4}{|c|}{$\begin{array}{c}\text { Table VI.3 } \\
\begin{array}{c}\text { Spherical Harmonic Fits }- \text { Independent Fits } \\
\text { milli-Kelvin }\end{array}\end{array}$} \\
\hline $\begin{array}{l}\text { Fitting Function } P_{i}^{\prime \prime} \\
1 \\
\sin \delta \\
\cos \delta \cos \alpha \\
\cos \delta \sin \alpha\end{array}$ & $\begin{array}{rr}\text { Q Fil } & \\
& 0.00 \\
-0.02 \\
0.02 \\
0.00\end{array}$ & $\begin{array}{ll}\text { U Fit } & \\
& -0.01 \\
& -0.03 \\
& 0.02 \\
& 0.08\end{array}$ & $\begin{array}{l}\text { Error } \\
0.03 \\
0.04 \\
0.05 \\
0.05\end{array}$ \\
\hline$\frac{1}{2}\left(3 \sin ^{2} \delta-1\right)$ & -0.02 & -0.05 & 0.06 \\
\hline $\begin{array}{l}\cos 2 \delta \cos \alpha \\
\cos 2 \delta \sin \alpha \\
\cos ^{2} \delta \cos 2 \alpha \\
\cos ^{2} \delta \sin 2 \alpha\end{array}$ & $\begin{array}{r}-0.05 \\
-0.03 \\
0.08 \\
-0.10\end{array}$ & $\begin{array}{r}0.01 \\
0.02 \\
-0.06 \\
0.15\end{array}$ & $\begin{array}{l}0.04 \\
0.04 \\
0.06 \\
0.06\end{array}$ \\
\hline$\frac{1}{2}\left(5 \sin ^{3} \delta-3 \sin \delta\right)$ & 0.04 & -0.03 & 0.08 \\
\hline$\frac{1}{4} \cos 6\left(5 \sin ^{2} 6-1\right) \cos \alpha$ & -0.02 & 0.01 & 0.05 \\
\hline$\frac{1}{4} \cos \delta\left(5 \sin ^{2} \delta-1\right) \sin \alpha$ & -0.06 & 0.01 & 0.05 \\
\hline $\begin{array}{l}\cos 2 \delta \sin \delta \cos 2 \alpha \\
\cos 2 \delta \sin \delta \sin 2 \alpha \\
\cos ^{3} \delta \cos 3 \alpha \\
\cos ^{3} \delta \sin 3 \alpha \\
\end{array}$ & $\begin{array}{r}0.03 \\
-0.05 \\
-0.01 \\
-0.07 \\
\end{array}$ & $\begin{array}{l}0.01 \\
0.08 \\
0.06 \\
0.04 \\
\end{array}$ & $\begin{array}{l}0.05 \\
0.05 \\
0.07 \\
0.08 \\
\end{array}$ \\
\hline 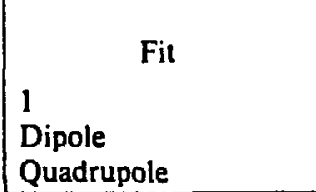 & $\begin{array}{c}Q: \frac{\chi^{2}}{D O F} \\
279 / 263 \mathrm{CL}=24 \% \\
279 / 261 \mathrm{CL}=21 \% \\
274 / 259 \mathrm{CL}=25 \%\end{array}$ & $\begin{array}{c}U: \frac{\chi^{2}}{D O F} \\
279 / 263 \mathrm{CL}=24 \% \\
281 / 261 \mathrm{CL}=19 \% \\
296 / 259 \mathrm{CL}=6 \%\end{array}$ & \\
\hline
\end{tabular}

discussed in appendix $E$ gives a definite prediction as to the functional form of $Q$ and $U$ for a model of anisotropic expansion with an axis of symmetry (Nanos 1979). Table VI.4 summarizes the best fit to this model. The model is a fairly good fit to the data but it is not significant. We have found no evidence for linear polarization over any of the areas surveyed.

\section{VI.4 Comparison to Previous Measurements}

There have been two previous measurements of the polarization of the CBR, Nanos (1979) and Caderni et al (1978), both with null results. Nanos performed an experiment similar to this one in 1973 at a wavelength of $32 \mathrm{~mm}$ for one declination $\delta=40^{\circ}$. Caderni et al used an balloon-borne infrared spectrometer operating at a wavelength of $0.5-3 \mathrm{~mm}$, but were forced to terminate after four hours of data taking. Because of the limiled sky coverage in both 


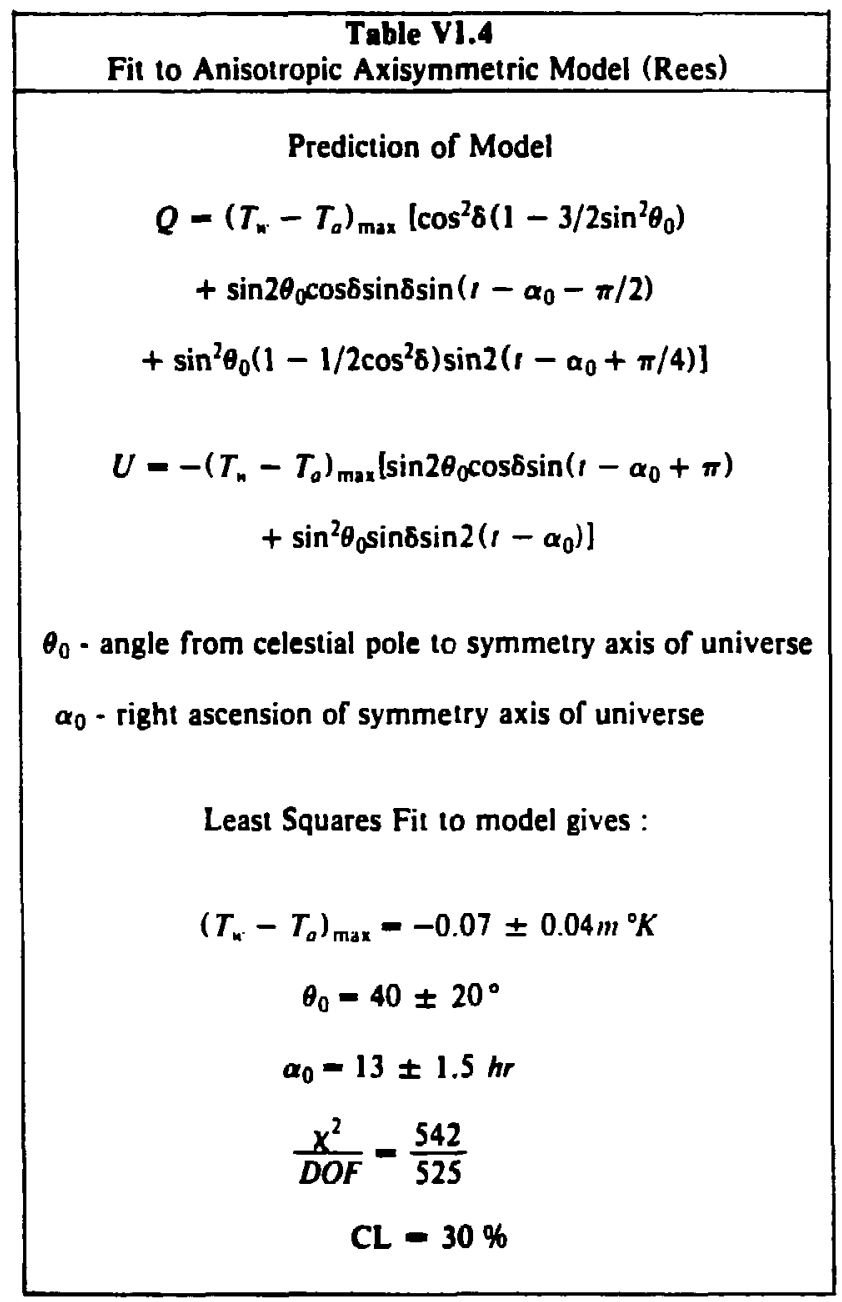

experiments, these data were not fit to spherical harmonics. The results of Nanos and Caderni et al are summarized in Table V1.5. Nanos's data shows a significant $(5 \sigma)$ average value for $Q$ and $U$ although this was interpreted as being caused by sidelobe pickup from a nearby building. The work described here represents about an order of magnitude improvement over previous measurements. 


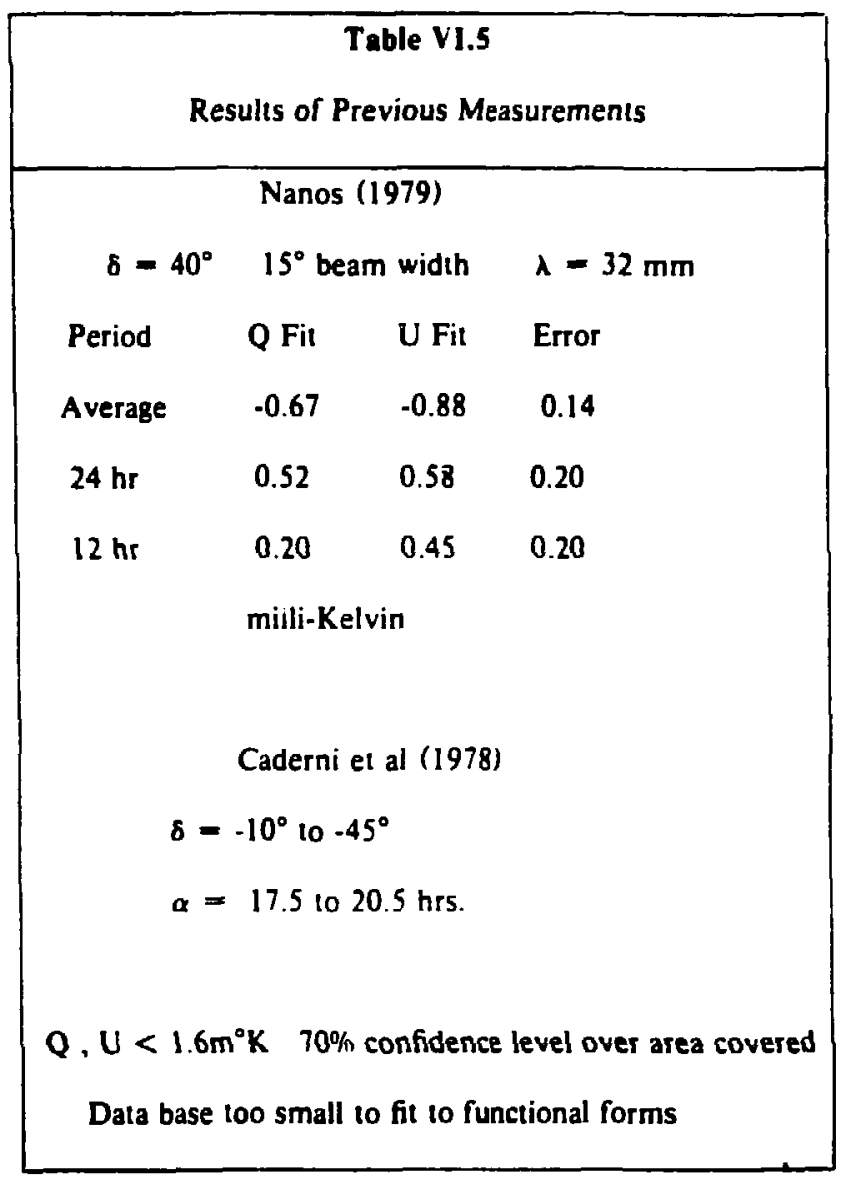




\section{Chapter VII - Astrophysical Interpretation}

\section{V11.1 LImits on Anisotropic Models}

The polarization limits obtained can be used to set limits on the types of models useful to describe the universe, as well as processes which can occur. In general, any model which produces an intrinsic intensity anisotropy in the background radiation, also will produce a polarization. Exactly what is meant by intrinsic requires some clarification. Intrinsic anisotropy here means that which is not produced by our particular frame of reference and which is present prior to the time of decoupling. Examples of intrinsic anisotropies include rotation of the universe and anisotropic expansion. Examples of anisotropies which are not intrinsic are local inhomogeneities (masses), local gravity waves, and the motion of our galaxy. These latter anisotropies would not be expected to produce any polarization. As stated before, one advantageous feature of this experiment is that it is only sensitive to intrinsic anisotropies; any perturbations present in the intensity which are simply due to our peculiar reference frame are not present and thus need not be subtracted away.

The degree of polarization induced by a given intrinsic anisotropy depends on the time at which decoupling occurred, since this sets the time scale on which matter and radiation interact. More specifically, the polarization depends on the ionization fraction as a function of time. Two cases will be considered in this regard. In case I, decoupling occurs at a $Z$ of 1500 with no reionization at later times. In case II, decoupling occurs at a $\mathrm{Z}$ of 1500 , but matter is reionized later at a $\mathbf{Z}$ of 7 , possibly corresponding to the era of early galaxy formation when it is postulated that radiation from the forming galaxies may be intense enough to reionize matter in the universe. In both cases, the calculation of Peebles (1968) was used for the ionization fraction through the era of decoupling (Negroponte and Silk, 1980), and it is assumed that the density is the critical density. Table VII.1 gives the limits that the polarization measurement places on various models and processes in terms of the two cases mentioned. 


\begin{tabular}{|c|l|l|}
\hline \multicolumn{3}{|c|}{ Table VII.1 } \\
\hline Model Constraints From Polarization Data \\
\hline \multirow{2}{*}{ Anisotropic Expansion } & $\frac{\delta h_{0}}{h_{0}}<6 \times 10^{-8}$ & Case 2 (reheat z=7) \\
Rotation of Universe & $\omega_{0}<10^{-10} \mathrm{sec}$-arc / century & $\frac{\delta h_{0}}{h_{0}}<2 \times 10^{-8}$ \\
Density Fluctuations & $\frac{\delta \rho}{\rho}<8 \times 10^{-3}$ & \\
& & \\
\hline
\end{tabular}

The calculations of Negroponte and Silk (1980) are used for the limits on anisotropic expansion (Appendix E). The calculations of Collins and Hawking (1973) are used for limits on the rotation of the universe along with the work of Negroponte and Silk to compute the polarization fraction. Adiabatic fluctuations are assumed for the limit on density inhomogeneities. In this case there is a simple relationship between the density fluctuations $\frac{\Delta p}{\rho}$ and the temperature fluctuations $\frac{\Delta T}{T}$; i.e. $\frac{\Delta T}{T}=\frac{1}{3} \frac{\Delta \rho}{\rho}$. The work of Negroponte and Silk is again used to calculate the polarization fraction.

\section{VI1.2 Comparison to Intensity Measurements}

The best limits on the intensity anisotropy other than the first order (motion) anisotropy come from the Princeton and Berkeley anisotropy experiments. The measured value of the first order term and limits on the higher order terms are shown in Table VII.2.

A direct comparison between the polarization and intensity measurements is not possible without a model to connect these two intrinsically different measurements. Such a model was first proposed by Rees (1968) and is discussed more fully in Appendix E. Its basis is that Thomson scattering of anisotropic radiation yields polarized radiation. As mentioned in the previous section, the basic input parameter of this model is the ionization fraction of matter as a function of time, or equivalently the matter temperature evolution of the universe. Utilizing 
the results of Appendix $E$, the comparison between polarization and intensity is given in Table Vll.3 for the case of an axisymmetric anisotropic universe.

\begin{tabular}{|crrrrr|}
\hline \multicolumn{6}{|c|}{ Table VII.2 } \\
Comparison To Intensity Measurements \\
Dipole and Quadrupole Fit - $\mathrm{m}^{\circ} \mathrm{K}$ \\
\hline \multicolumn{5}{|c}{ Intensity } & \multicolumn{3}{c|}{ Polarization } \\
\hline Fitting Function & Fit & Error & Q Fit & U Fit & Error \\
\hline $\cos \delta$ & -0.18 & 0.39 & -0.02 & -0.03 & 0.04 \\
$\cos \delta \cos \alpha$ & -2.78 & 0.28 & 0.02 & 0.02 & 0.05 \\
$\cos \delta \sin \alpha$ & 0.66 & 0.29 & 0.00 & 0.08 & 0.05 \\
$\frac{1}{2}\left(3 \sin ^{2} \delta-1\right)$ & 0.38 & 0.26 & -0.02 & -0.05 & 0.06 \\
$\sin 2 \delta \cos \alpha$ & -0.34 & 0.29 & -0.05 & 0.01 & 0.04 \\
$\sin 2 \delta \sin \alpha$ & 0.02 & 0.24 & -0.03 & 0.02 & 0.04 \\
$\cos ^{2} \delta \cos 2 \alpha$ & -0.11 & 0.16 & 0.08 & -0.06 & 0.06 \\
$\cos ^{2} \delta \sin 2 \alpha$ & 0.06 & 0.20 & -0.10 & 0.15 & 0.06 \\
\hline
\end{tabular}

-( Smoot and Lubin 1979)

\begin{tabular}{|c|c|}
\hline \multicolumn{2}{|c|}{$\begin{array}{c}\text { Table VII.3 } \\
\text { Comparison of Polarization and Intensity } \\
\end{array}$} \\
\hline Case & $\frac{P}{C}=$ ratio of pol. $10 \mathrm{int}$. \\
\hline No reheat of plasma & 0.04 \\
\hline $\begin{array}{c}\text { Reheat at } 2=7 \\
\boldsymbol{\Omega}_{H}=1 \\
\boldsymbol{\Omega}_{H}=0.1\end{array}$ & $\begin{array}{l}0.4 \\
0.07\end{array}$ \\
\hline Reheat at $2=40 \Omega_{H^{-1}}$ & 2 \\
\hline Reheat at $Z=100 \Omega_{H}-1$ & 0.5 \\
\hline
\end{tabular}




\section{Appendix A - Wobble Correction}

When the instrument is tilted away from the local vertical, the gravitational torque on the radiometer causes stress on the components. This leads to a modulated offset with a period the same as the rotation period. A true polarized signal would have a period which is one half of the rotation period. Simply rotating by a full $360^{\circ}$ cycle in $45^{\circ}$ steps would appear to allow complete cancellation of this effect. However, there is a residual second order effect on the order of one percent apparently caused by the mechanical asymmetry of construction which adds a constant level to both $Q$ and $U$. The mechanical nature of this wobble has been verified by physically rotating the instrument by $180^{\circ}$ about its axis and noting the $D C$ level of $Q$ and $U$ reversed sign.

For the northern hemisphere runs, the typical wobble correction is a few tenths of a millidegree Kelvin. For the southern hemisphere data, the instrument was in a different configuration and for the data at $8=-37^{\circ}$, a bolt worked loose causing a false polarized signal of about a millidegree Kelvin. It is important to note that this correction is only to the average level and does not effect the time dependence of the data.

To make the correction, the northern and southern hemisphere data were analyzed separately. A least squares fit is made to the wobble versus DC level assuming a linear relationship forcing the fit through the origin ( 0 wobble $=0$ correction). This fit is done separately for $\mathbf{Q}$ and $\mathbf{U}$ for the northern hemisphere runs. A linear relationship is expected because of the mechanical nature of the effect. The data and best fit is shown in Figure A.1. The correction is summarized in Table A.1. 


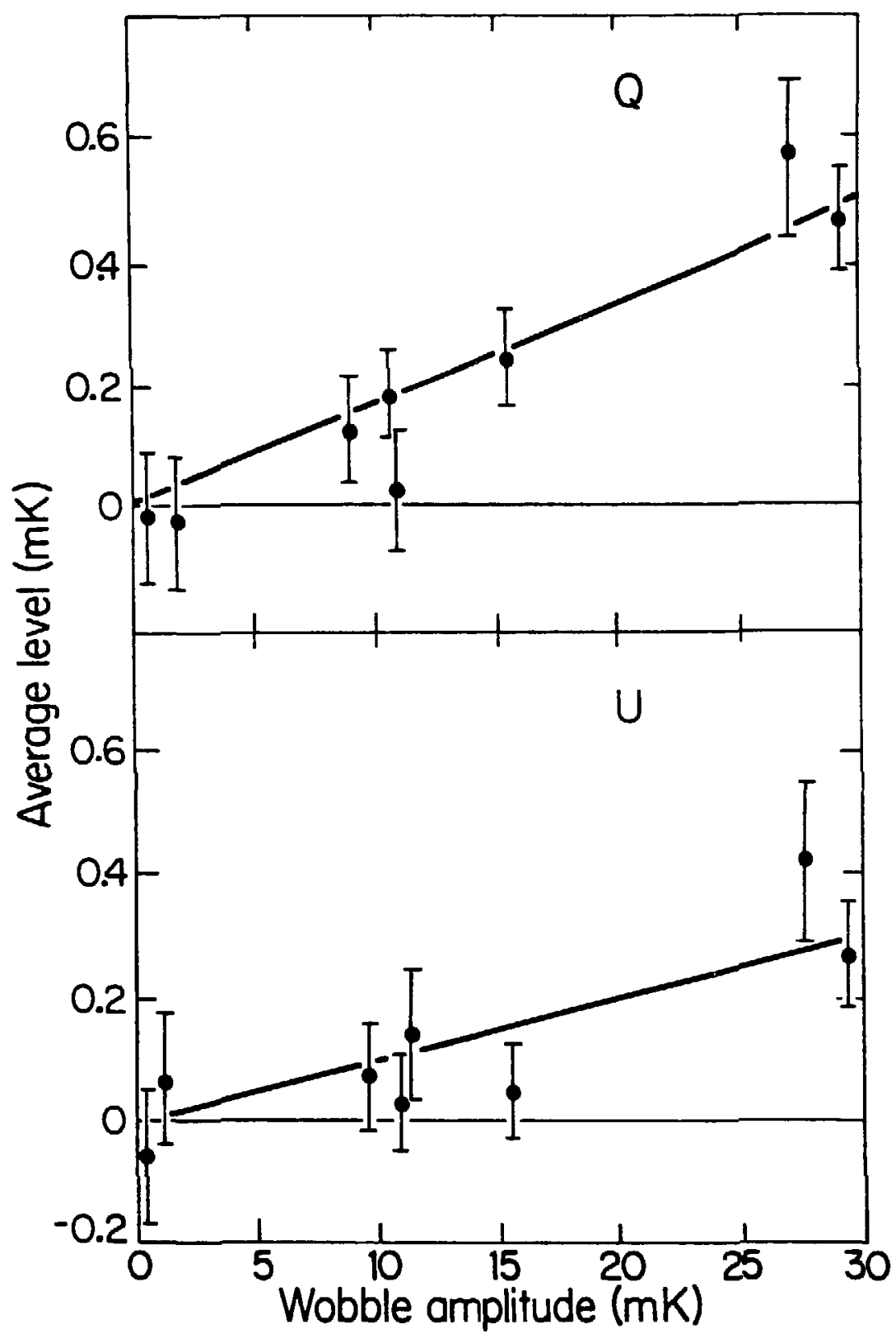

XBL802-238

Figure A.1 - Wobble Amplitude Versus DC Level 


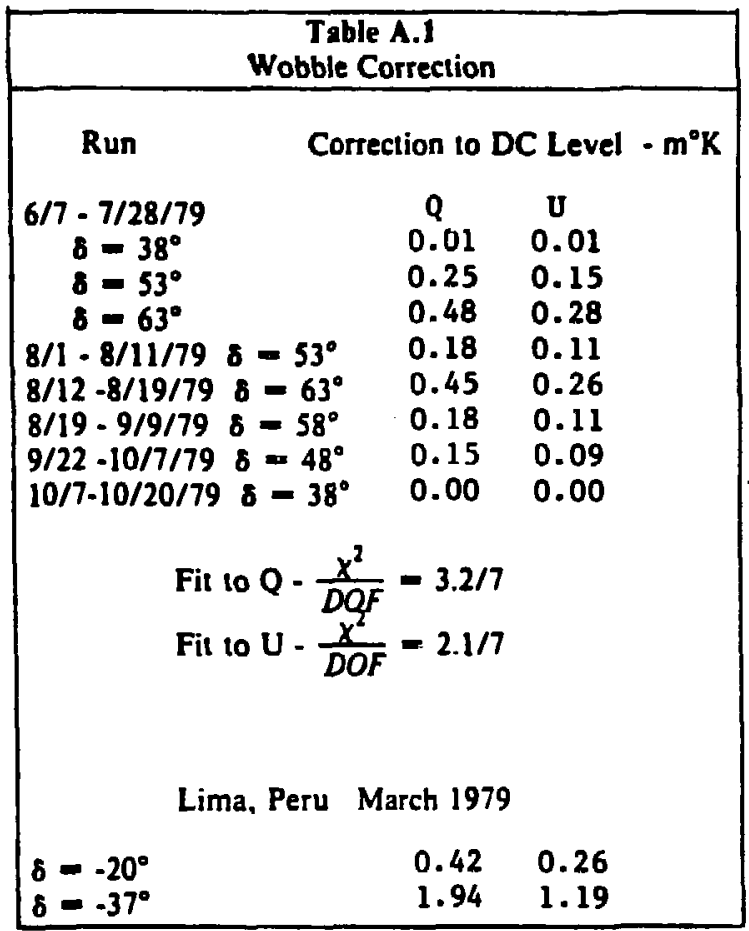




\section{Appendix B - Microwave Circuit}

\section{B.1 General Description}

The microwave circuit is shown in Figure B.1. It consists of a very low sidelobe antenna which accepts all polarization states, a Faraday Rotation Switch to switch between polarization states to be accepted, a superheterodyne microwave receiver and a lockin amplifer whose output is proportional to the power difference in polarization states selected by the Faraday Rotation Switch.

\section{B.2 Antenna}

The antenna used is a state-of-the-art dual mode scalar horn developed specifically to have very good sidelobe rejection (Janssen et al, 1979). An additional benefit is the symmetry in the response pattern about the axis of the horn. The measured tesponse pattern is shown in Figure B.2 for orthogonal passes across the antenna as a function of angle from the antenna axis. Also shown is the response pattern with a mock-up of the ground shield used. The beam has a FWHM of about $7^{\circ}$ with a gain of $27.8 \mathrm{db}$ at $33 \mathrm{GHz}$. It has an insertion loss of $0.1 \mathrm{db}$ and a VSWR of 1.1 .

The beam pattern and ground shield measurements were taken at JPL (Janssen et al, 1979). A digitized version of these patterns indicates that the sidelobe pickup from the ground should induce a constant apparently polarized signal of magnitude less than $0.1 \mathrm{~m}^{\circ} \mathrm{K}$ for tilt angles less than 25 degrees. However due to the uncertainty in the beam pattern measurements and incomplete knowledge of the azimuthal dependence of the beam pattern, this limit may be incorrect by a factor of two. 


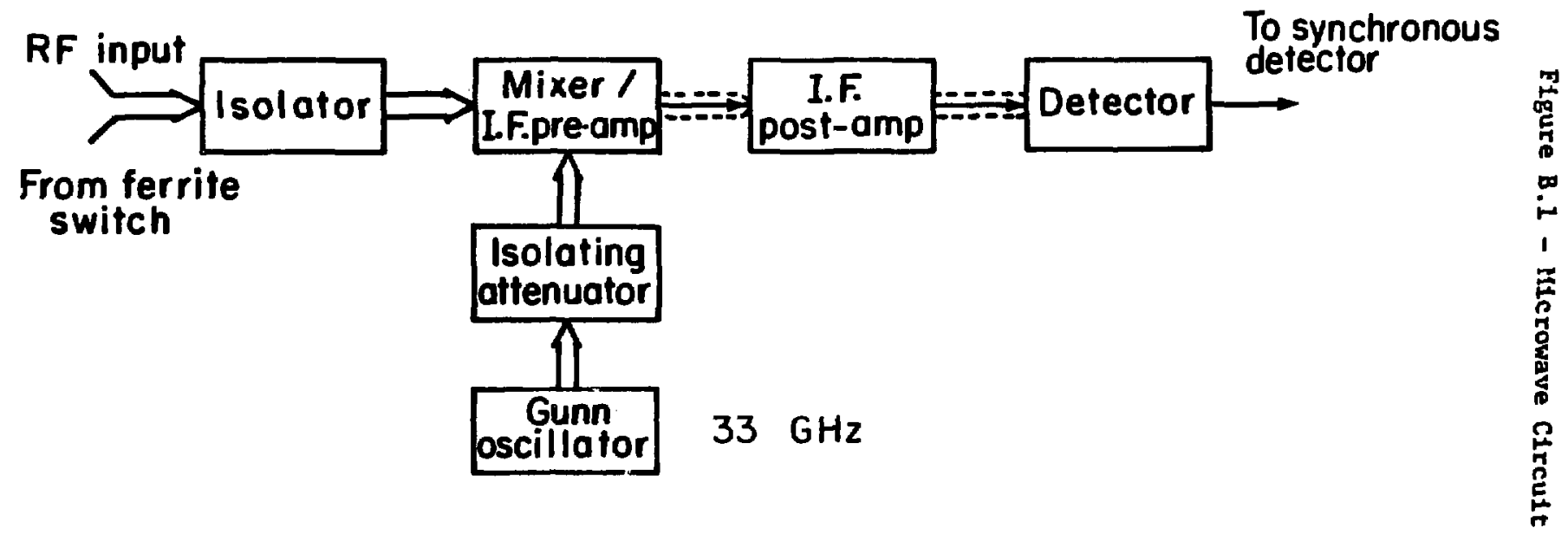

XBL776-I $339 A$ 
Figure B.2a - Measured Antenna Patterns

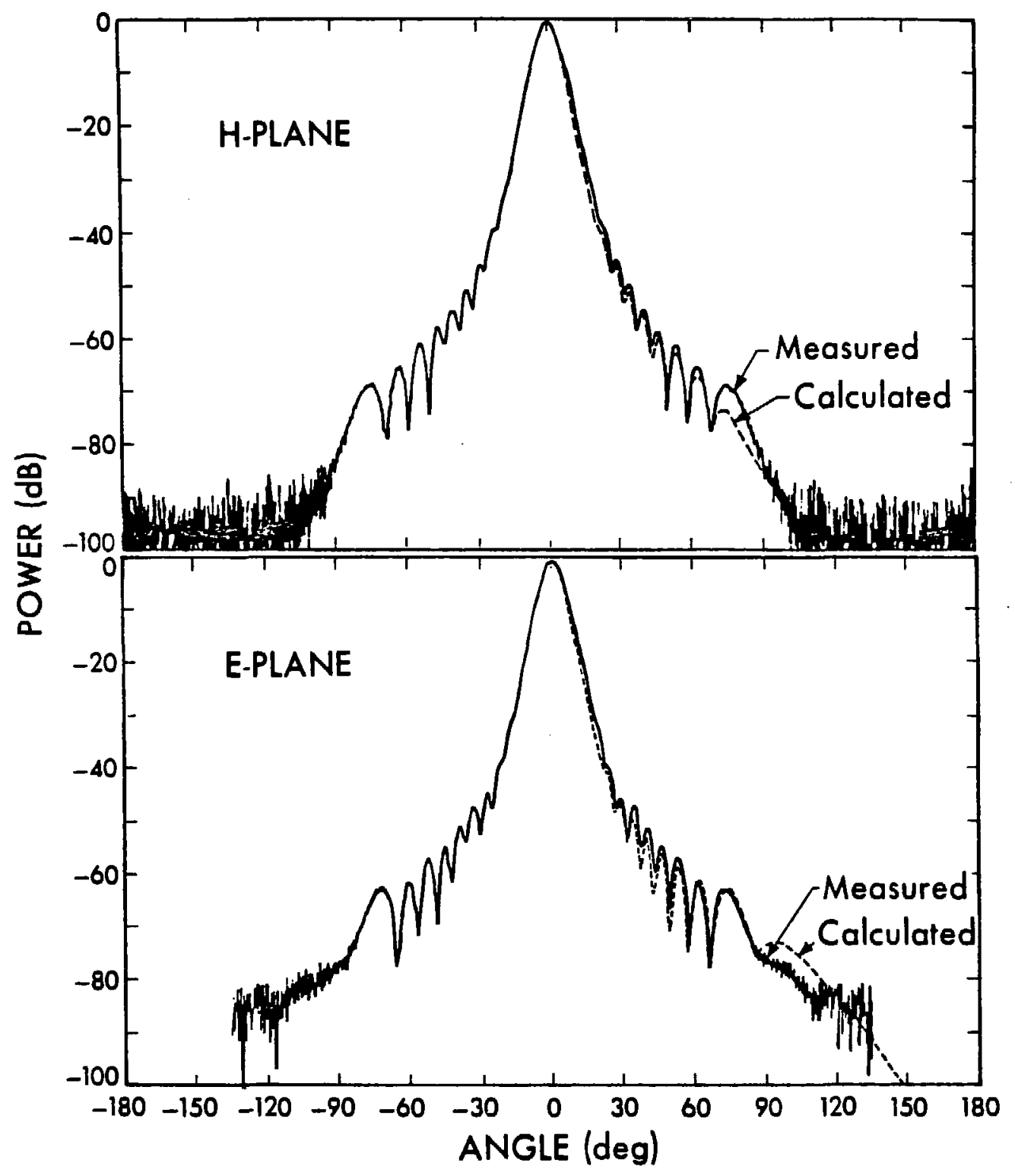

XBL $778-1809$ 

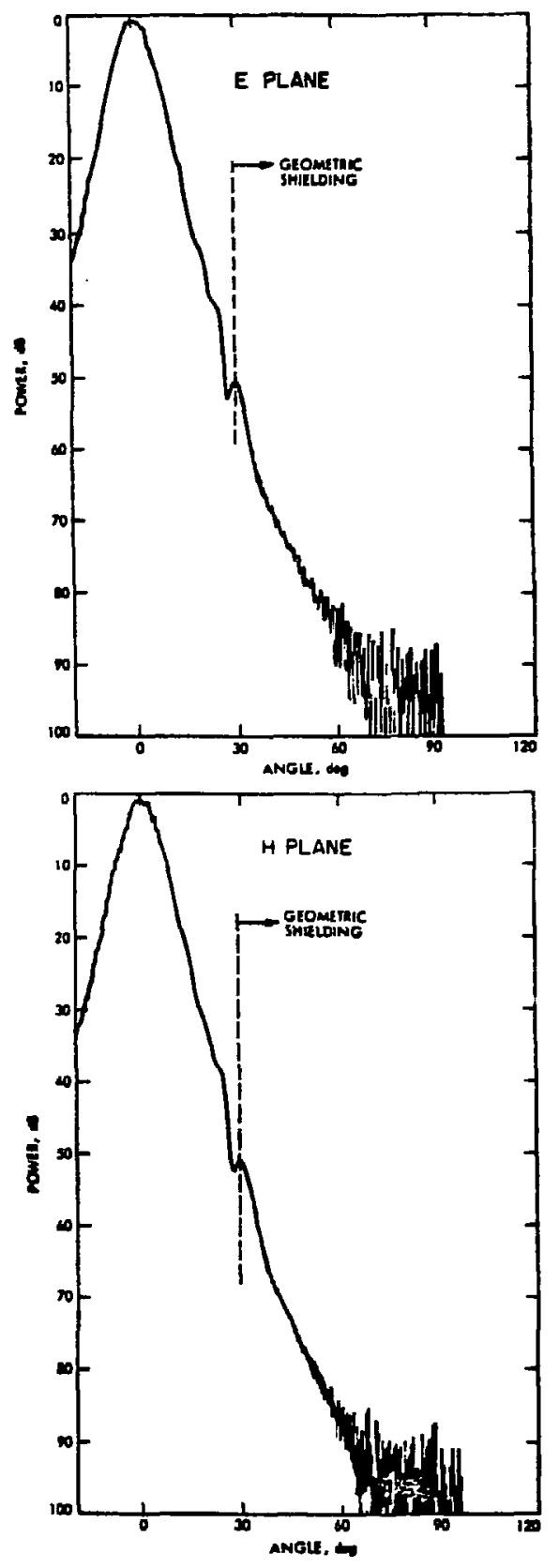

ne renas 4 


\section{B.3 Faraday Rotation Switch}

The switch was manufactured for us by TRG-Alpha of Wobum, Massachuselts. It consists of a ferrite "toothpick" embedded in teflon surrounded by a solenoidal winding. It is shown schematically in Figure B.3. For discussion, the ferrite can be thought of as a collection of magnetic moments. Application of an axial magnetic field causes the ferrite to become birefringent, meaning that photons of different helieities travel at different phase speeds through the ferrite (Yariv, 1967; Gurevich, 1960). As linearly polarized radiation can be thought of as equal components of right and left circularly polarized radiation with a definite phase relation, it is seen that transmission through the ferrite simply rotates the plane of polarization. Reversing the magnetic fields causes rotation of the opposite sense. By suitable arrangemeni of magnetic field strength and ferrite length, any desired rotation angle can be arranged. In our case a current of \pm 50 ma causes a rotation of $\pm 45^{\circ}$.

A transition piece between the switch and the receiver allows radiation linearly polarized along a prescribed direction to pass while reflecting the orthogonal component. So by switching alternately $\pm 45^{\circ}$ we alternately accept orthogonal polarization states at the receiver. This is analogous to rotating a polaroid sheet by $\pm 45^{\circ}$. In our case we switch at $100 \mathrm{~Hz}$.

\section{B.4 Receiver}

The receiver is a balanced mixer pumped by a Gunn effect oscillator. The mixer was built for us by Spacecom Inc. of Goleta, California. It has a center frequency of $33.0 \mathrm{GHz}$ and an IF bandwidth of $500 \mathrm{MHz}$. Originally the receiver utilized Schottky barrier silicon mixing diodes and gave an overall system ternperature of $900^{\circ} \mathrm{K}$.

The mixer was overhatled in the fall of 1978. Through the use of GaAs diodes, the system temperature was reduced to about $500^{\circ} \mathrm{K}$. A low frequency spectral analysis of the original mixer (silicon diodes) showed the "1/f knee" is around $1 \mathrm{~Hz}$, so switching at $100 \mathrm{~Hz}$ is sufficient to overcome $1 /[$ noise. The test was done with little thermal regulation so the $1 / f$ knee is probably even lower in the system being used. 


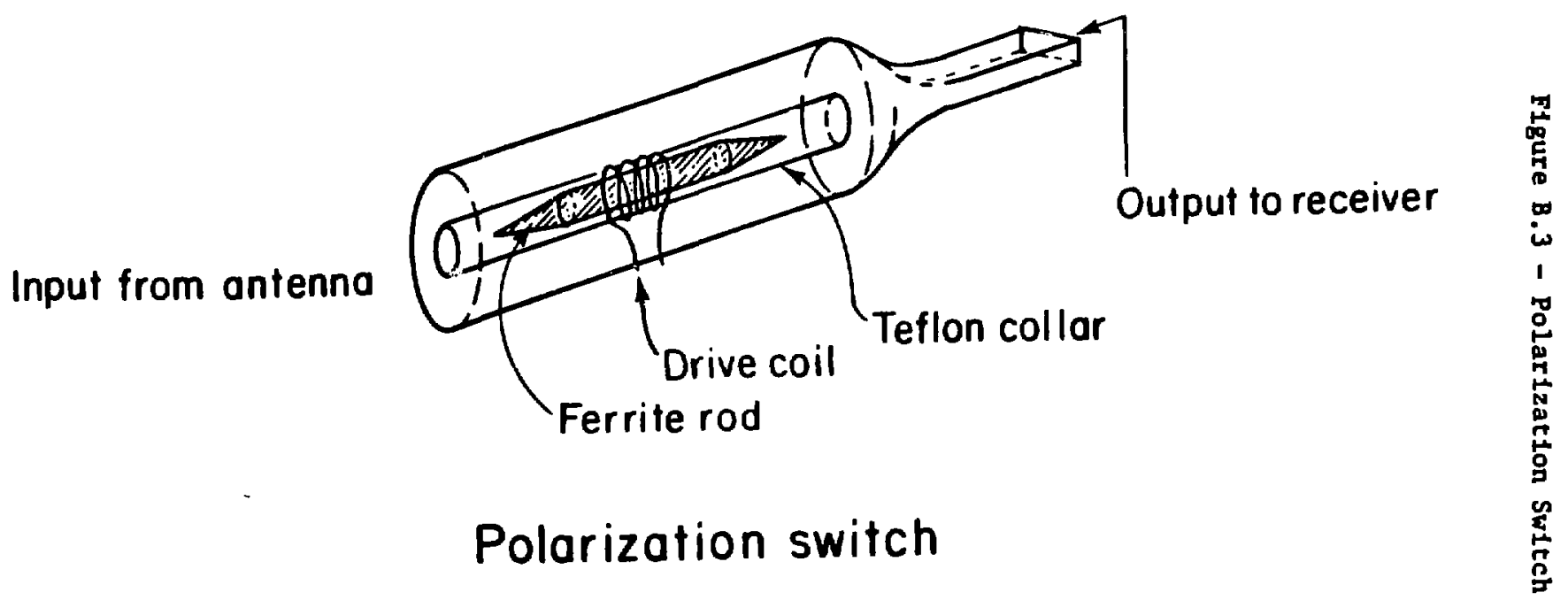

XBL 7910-4286 


\section{Appendix C - Systematic Error Analysis}

\section{C.I Introduction}

The detection of very low signal levels requires a system with excellent gain stabitity. The stability required of a total power radiometer with a noise temperature of $500^{\circ} \mathrm{K}$, capable of detecting a $0.1 \mathrm{~m}^{\circ} \mathrm{K}$ signal is:

$$
\frac{\text { minimum delectable signal }}{\text { syslem noise temperature }}=\frac{0.1 \mathrm{~m}^{\circ} \mathrm{K}}{500^{\circ} \mathrm{K}}=2 \times 10^{-7}
$$

Such stability would be extremely difficult to achieve, especially on the time scale required for integration to $0.1 \mathrm{~m}^{\circ} \mathrm{K}$, which is approximately one week.

A Dicke-switched radiometer greatly reduces this requirement of stability by rapidly switching the radiometer input between two signal states and taking their difference. The gain stability requirement is then reduced by the ratio of the system noise temperature to the offset temperature which is typically less than $50 \mathrm{~m}^{\circ} \mathrm{K}$. Thus the gain stability required becomes:

$$
\frac{\text { minimum detectoble signal }}{\text { offset temperalure }}=\frac{0.1 \mathrm{~m}^{\circ} \mathrm{K}}{50 \mathrm{~m}^{\circ} \mathrm{K}}=2 \times 10^{-3}
$$

This stability is only required for times of the order of the polarimeter cycle time (minutes). It can be seen that the smaller the offset is, the less stringent the gain stability requirement becomes.

The total radiometer gain is made up of $10^{6}$ power gain in the receiver (mixer and IF) plus $10^{6}$ valtage gain in the lockin amplifier. 
The polarimeter can be conveniently divided into three parts for thermal analysis. They are:

(1) Microwave Front End: ground shield, antenna, polarization switch, and isolator.

(2) Receiver: mixer-preamp, IF, L.O., and detector diode

(3) Post Electronics: lockin amplifier (synchronous detector) analog to digital converter (A/D), and power supplies.

\section{C.2 Microwave Front End}

Ofisets due to asymmetries in the instrumental response to differing polarization states are one of the primary diffculties in experiments of this type. Offsets which vary synchronously with the polarimeter rotation are indistinguishable from a real signal. Ofisets not synchronous with the polarimeter rotation can be reduced by sufficient data taking.

The signal at the receiver input for one polarization state is:

$$
\begin{aligned}
T_{S}= & \left(b\left(\alpha T_{C B}+(1-\alpha) T_{A T M}\right)+a E_{S} T_{S}+a{ } E_{G} T_{G}\right) A P I \\
& +(1-A) T_{A} P I+(1-P) T_{P S} I+(1-I) T_{I}+p T_{I}
\end{aligned}
$$

The parameters and their values are listed in Table C.1. 


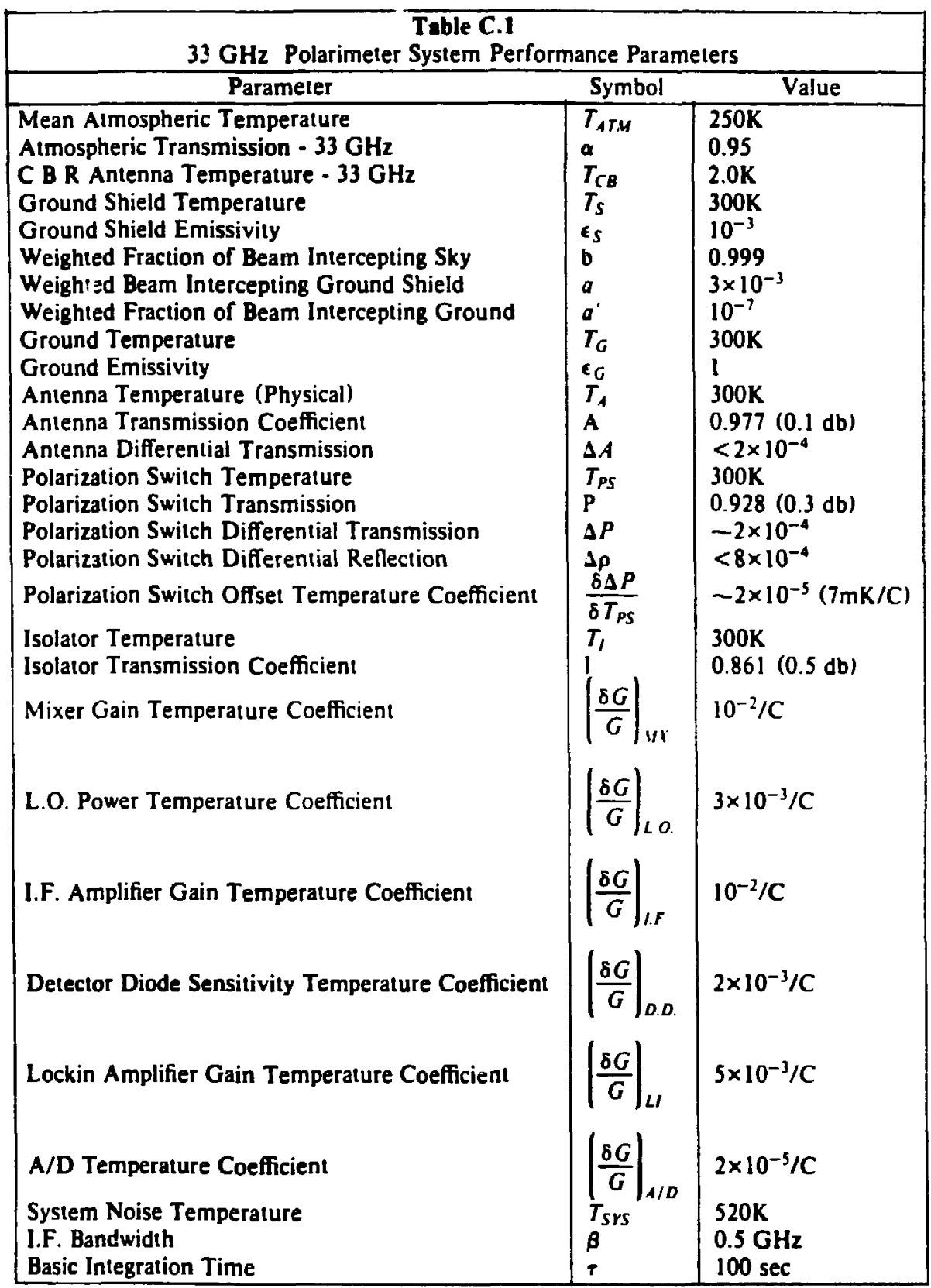


The differential signal (offset) between the two polarization states is given by:

$$
\begin{aligned}
S=\Delta T_{S}= & {\left.\left[\Delta\left(b \alpha T_{C B}\right)+\Delta\left(b(1-\alpha) T_{A T M}\right)+\Delta\left(a e_{S} T_{S}\right)+\Delta\left(a^{\prime} \epsilon_{G} T_{G}\right)\right] A P\right] } \\
& +\left[b\left(\alpha T_{C B}+(1-\alpha) T_{A T M}\right)+a \epsilon_{S} T_{S}+a^{\prime} \epsilon_{G} T_{G}-T_{A}\right] P I \Delta A \\
& \left.\left.+\left[\left(b\left(\alpha T_{C B}+(1-\alpha) T_{A T M}\right)+a \epsilon_{S} T_{S}+a^{\prime} \epsilon_{G} T_{G}\right) A\right]+(1-A) T_{A}\right]-T_{P S} I\right] \Delta P \\
& +T_{1} \Delta \rho
\end{aligned}
$$

If the physical temperatures of the various parameters are now allowed to vary, the change in offset with temperature is given by:

$$
\begin{aligned}
\delta S= & {\left[\Delta\left(b T_{C B} \delta \alpha\right)+\Delta\left(b \delta\left((1-\alpha) T_{A T M}\right)\right)+\Delta\left(a \epsilon_{S} \delta T_{S}\right)+\Delta\left(a^{\prime} \epsilon_{G} \delta T_{G}\right)\right] A P I } \\
& +\left[b T_{C B} \delta \alpha+b \delta\left((1-\alpha) T_{A T M}\right)+a \epsilon_{S} \delta T_{S}+a^{\prime} \epsilon_{G} \delta T_{G}-\delta T_{A}\right] P I \Delta A \\
& +\left[\left(b T_{C B} \delta \alpha+b \delta\left((1-\alpha) T_{A T I I}\right)+a \epsilon_{S} \delta T_{S}+a^{\prime} \epsilon_{G} \delta T_{G}\right) A I+(1-A) / \delta T_{A}-I \delta T_{P S S}\right] \Delta P \\
& +\left[\left(b\left(\alpha T_{C B}+(1-\alpha) T_{A T M}\right)+a \epsilon_{S} T_{S}+a^{\prime} \epsilon_{G} T_{G}\right) A I+(1-A) I T_{A}-T_{P S} I\right] \frac{\delta \Delta P}{\delta T_{P S}} \delta T_{P S}
\end{aligned}
$$

$$
+\Delta \rho \delta T_{1}
$$


The equivalent change in signal referred to the input of the antenna is given by:

$$
\begin{aligned}
\frac{\delta S}{A P I}- & {\left[\left(T_{C B}-T_{A T M}\right) \Delta b+\left(T_{C B-T_{A T M}}\right) b \frac{\Delta A}{A}+\left(T_{C B}-T_{A T M}\right) b \frac{\Delta P}{P}\right] \delta \alpha } \\
& +\left[\Delta\left(a^{\prime} \epsilon_{G}\right)+a^{\prime} \epsilon_{G} \frac{\Delta A}{A}+a^{\prime} e_{G} \frac{\Delta P}{P}\right] \delta T_{G} \\
& +\left[\Delta\left(a e_{S}\right)+a e_{S} \frac{\Delta A}{A}+a e_{S} \frac{\Delta P}{P}\right] \delta T_{S} \\
& +\left[\frac{1-A}{A} \frac{\Delta P}{P}-\frac{\Delta A}{A}\right] \delta T_{A} \\
& +\left[\left(b\left(\alpha T_{C B}+(1-\alpha) T_{A T M}\right)+a \epsilon_{S} T_{S}+a^{\prime} \epsilon_{G} T_{G}+\frac{1-A}{A} T_{A}-\frac{T_{P S}}{A}\right) \frac{1}{P} \frac{\delta \Delta P}{\delta T_{P S}}-\frac{1}{A} \frac{\Delta P}{P}\right] \delta T_{P S} \\
& +\frac{\Delta \rho}{A P I} \delta T_{1}
\end{aligned}
$$

where the parameters have been rearranged to show the dependence on temperature variation. The goal of the thermal design of the experiment is to reduce this change in offset to below 0.1 $\mathrm{m}^{\circ} \mathrm{K}$ for changes synchronous with the polarimeter.

Using the parameter values of Table C.I gives the following. where the first term representing the atmospheric variation has been ignored for the moment.

$$
\frac{\delta S}{A P I}<10^{-7} \delta T_{G}+3 \times 10^{-6} \delta T_{S}+2 \times 10^{-4} \delta T_{A}+6 \times 10^{-3} \delta T_{P S}+10^{-3} \delta T_{1}
$$

This assumes a conspiracy where all effects add in magnitude and as such is a worst case analysis.

The active heater circuits, along with the thermoelectric cooler, typically regulate the polarimeter $100.1{ }^{\circ} \mathrm{C}$ for time periods of the order of hours. This is valid except during periods of extreme heat input, such as during very warm days $\left(\mathrm{T}>30^{\circ} \mathrm{C}\right)$ which fortunately were infrequent.

The quantity of interest here is the temperature variation of the system components dur- 
ing the polarimeter rotation, in which alternate positions are 200 seconds apart. Table C. 2 gives the temperature variation of the various instrument components during the rotation cycle obtained from temperature sensors at critical points.

\begin{tabular}{|l|l|l|}
\hline \multicolumn{3}{|c|}{ Table C.2 } \\
Temperature Stability of Components over Alternate Rotations - 200 sec. \\
\hline \multicolumn{1}{|c|}{ Component } & \multicolumn{1}{|c|}{ Symbol } & \multicolumn{1}{|c|}{ Value - ${ }^{\circ} \mathrm{C}$} \\
Ground & $\delta T_{G}$ & $<10$ \\
Ground Shield Temperature & $\delta T_{S}$ & $<10$ \\
Antenna Temperature & $\delta T_{A}$ & $<0.1$ \\
Polarization Switch Temperature & $\delta T_{P S}$ & $<0.01$ \\
lsolator Temperature & $\delta T_{I}$ & $<0.05$ \\
Mixer Temperature & $\delta T_{M}$ & $<0.02$ \\
L.O. Temperature & $\delta T_{L . O .}$ & $<0.1$ \\
I.F. Amplifier Temperature & $\delta T_{I . F .}$ & $<0.02$ \\
Detector Diode Temperature & $\delta T_{D . D .}$ & $<0.02$ \\
Lockin Amplifier Temperature & $\delta T_{L I}$ & $<0.1$ \\
A/D temperature & $\delta T_{A / D}$ & $<2$ \\
\hline
\end{tabular}

Using the values of Table C. 2 the upper limit on the false signal induced by temparature variation is $\delta T<0.1 \mathrm{~m}^{\circ} \mathrm{K}$. Additionally this thermally induced signal is generally na: syath,ronous with the polarimeter rotation and will therefore tend to average out.

\section{C.3 Receiver and Post Detection Electronics}

A similar analysis applies to the receiver and downstream electronics. The magnitude of the final digitized signal $D$ is an overall gain constant $\mathbf{G}$ times the apparent signal $\mathbf{S}$ (ofiset plus real signal).

$$
D=G S
$$

The difference in this value caused by overall gain changes is

$$
6 D=(6 G) S
$$


The gain variation can be ascribed to a number of changes in system components such as changes in:
(1) mixer IF gain
(2) detecior diode sensitivity
(3) L.O. power
(4) lockin amplifier
(5) A/D gain

Assuming a conspiratorial effect (worst case analysis) gives:

$$
\frac{\delta D}{D}=\frac{\delta G}{G}-\left(\frac{\delta G}{G}\right)_{M X}+\left(\frac{\delta G}{G}\right)_{I F}+\left(\frac{\delta G}{G}\right)_{L O}+\left(\frac{\delta G}{G}\right)_{D D}+\left(\frac{\delta G}{G}\right)_{L I}+\left(\frac{\delta G}{G}\right)_{A: D}
$$

Inserting the values from Table $\mathrm{C} .1$ and converting to equivalent temperature gives:

$$
\begin{aligned}
\delta T_{\text {rearded }}<\left(10^{-2} \delta T_{M X}+10^{-2} \delta T_{I F}\right. & +3 \times 10^{-3} \delta T_{L O}+2 \times 10^{-3} \delta T_{D D} \\
& \left.+5 \times 10^{-3} \delta T_{L I}+2 \times 10^{-5} \delta T_{A / D}\right) S
\end{aligned}
$$

Inserting the values for the component temperature variation during a rotation cycle from Table C.2 and using an offset value of $50 \mathrm{~m}^{\circ} \mathrm{K}$ gives:

$$
8 T_{\text {recorded }}<0.06 m^{\circ} K
$$

In general as mentioned before, this variation is not synchronous with the polarimeter rotation and will average out.

\section{C.4 Atmospheric Fluctuations}

The first line of (C.4) represents the atmospheric contribution to the false signal.

$$
\delta T_{A T M}=\left[\left(T_{C B}-T_{A T M}\right) \Delta b+\left(T_{C B}-T_{A T M}\right) b \frac{\Delta A}{A}+\left(T_{C B}-T_{A T M}\right) b \frac{\Delta P}{P}\right] \delta \alpha
$$

The first term represents the asymmetry in the response of the antenna to the orthogonal polarization states. The second and third term represent the difierential insertion loss of the antenna 
and polarization switch (respectively) for the two orthogonal polarizations. Recall that " $\Delta$ " refers to differences between polarization states while " $\delta$ " refers to changes over the rotation cycle.

For example, a cloud will produce a small (apparently) polarized signal due to the slight differences in the gain of the antenna for the two orthogonal polarizations. This is manifest in the first term of (C.11). As an example of the second and third term a change in the overall opacity of the atmosphere will cause a change in the offset because of differential absorption by the switch and antenna for the two polarization states. This change in offset is equivalent to an apparent signal. It is important to note that in these processes there is no correlation to the rotation state of the polarization and thus with sufficient sampling this false signal should average out. However processes of the above mentioned type will increase the overall noise of the system.

Using the values of Table C.1 in (C.11) gives:

$$
\delta T_{A T M}<(250 \Delta b+0.1) \delta a
$$

A typical heavy cloud $\left(1 \mathrm{~g} / \mathrm{cm}^{2}\right.$ precipitable water ) would cause a change in opacity $6 \alpha$ of $10^{-2}$ while a typical weighted differential response might be $\Delta b \sim 10^{-3}$. This would cause a "signal" of about $4 \mathrm{mK}$ during a rotation cycle.

The system noise (RMS) during a cycle is about $2.7 \pm 0.1 \mathrm{mK}$ from receiver noise. Experimentally during a typical run the RMS fuctuations on $Q$ and $\mathcal{J}$ for a rotation cycle are about $3.5 \pm 0.2 \mathrm{mK}$ while data taken during "clear" days is consistent with receiver noise. There is no evidence to suggest this (occasional) extra atmospheric noise does not integrate down as random noise. 


\section{Appendix D - Backgrounds}

\section{D.1 Introduction}

As with most experiments dealing with nature there are backgrounds to be dealt with. There are two choices; either deal with the backgrounds and subtract them in the data analysis or try to design an experiment which avoids them. The latter was followed here. Possible backgrounds and interferences are listed in Table D.1.

\section{D.2 Galactic and Extragalactlc}

Diffuse galactic emission is dominated by synchrotron radiation from energetic electrons spiraling in galactic magnetic fields and by emission from ionized hydrogen (H-II). Synchrotron emission is significantly linearly polarized typically $10-50 \%$ while H-Il emission is not. So synchrotron emission is more relevant as a background. Figure D.1 shows an extrapolation of a low frequency $(400 \mathrm{MHz}$ ) full sky survey (Taylor, 1973) based upon high frequency measurements of the scaling law and averaged over our beam pattern (Witebsky, 1978). The scaling is essentially a power law in frequency; for equivalent antenna temperature $T_{A} \sim \nu^{-2.8}$ so the ernission is less at higher frequency and except in the galactic plane, emission is less than 0.3 $\mathrm{m}^{\circ} \mathrm{K}$. What is really of interest is the polarized component since unpolarized low level radiation is not a problem. Surveys at low frequency have been made which measure the polarization (Berkhuijsen, 1971; Brouw \& Spoelstra, 1976). Utilizing the $1411 \mathrm{MHz}$ polarization survey of Brouw and Spoelstra an estimation of the polarized emission was made at $33 \mathrm{GHz}$. Figure D.2 shows a contour map estimation based on their data. The extrapolation assumes $T_{A} \sim \nu^{-2.8}$ with errors likely to be no more than a factor of two. Because the beam pattern of the antenna is fairly broad, extragalactic sources are negligible at the $0.1 \mathrm{~m}^{\circ} \mathrm{K}$ for all known sources. 
Table D.1 - Posstble Systemat1c Errors : Causes and Soluttons

\section{PROBLEM}

\begin{tabular}{|c|c|c|}
\hline Receiver noise & $\begin{array}{l}\text { Integrate for sufficient } \\
\text { time }\end{array}$ & $60 \mathrm{mK} / \mathrm{sec}^{1 / 2}$ \\
\hline I/f noise & $\begin{array}{l}\text { Switch pol. states } \\
\text { at } 100 \mathrm{~Hz}\end{array}$ & - \\
\hline Temp. dep, of switch & Regulate temp. to $0.2^{\circ} \mathrm{C}$ & $<0.08 \mathrm{mk}$ \\
\hline Temp. dep. of I.F. & Regulate temp. & $<0.01 \mathrm{mk}$ \\
\hline $\begin{array}{l}\text { Mag. field dep. of } \\
\text { switch }\end{array}$ & Shield switch & $<0.08 \mathrm{mk}$ \\
\hline $\begin{array}{l}\text { Antenna sidelobe } \\
\text { pickup }\end{array}$ & $\begin{array}{l}\text { Use good antenna + } \\
\text { ground shield }\end{array}$ & $<0.02 \mathrm{mk}$ \\
\hline RF pickup. & RF shield apparatus & - \\
\hline Instrument asymmetry & $\begin{array}{l}\text { Rotate equipment at } \\
100 \mathrm{sec}\end{array}$ & - \\
\hline Galactic backgrounds & High freq. & $<0.05 \mathrm{mk}$ \\
\hline
\end{tabular}

XBL 799-3002A 
Figure D.1 - Estimated Galactic Synchrotron Radiation at $33 \mathrm{GHz}$

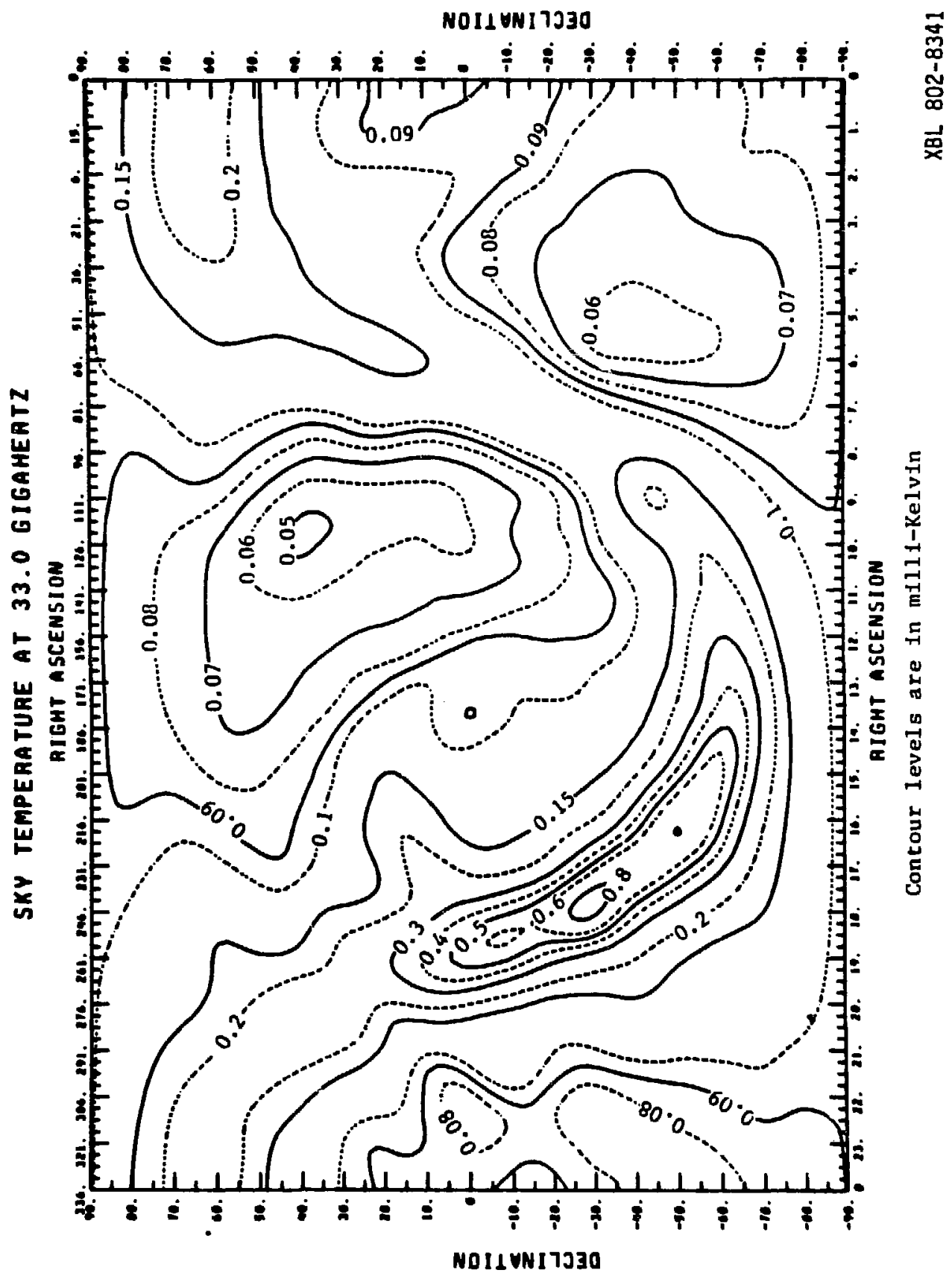


Figure D.2 - Estimated Polarized Galactic Emission at $33 \mathrm{GHz}$ micro-Kelvin
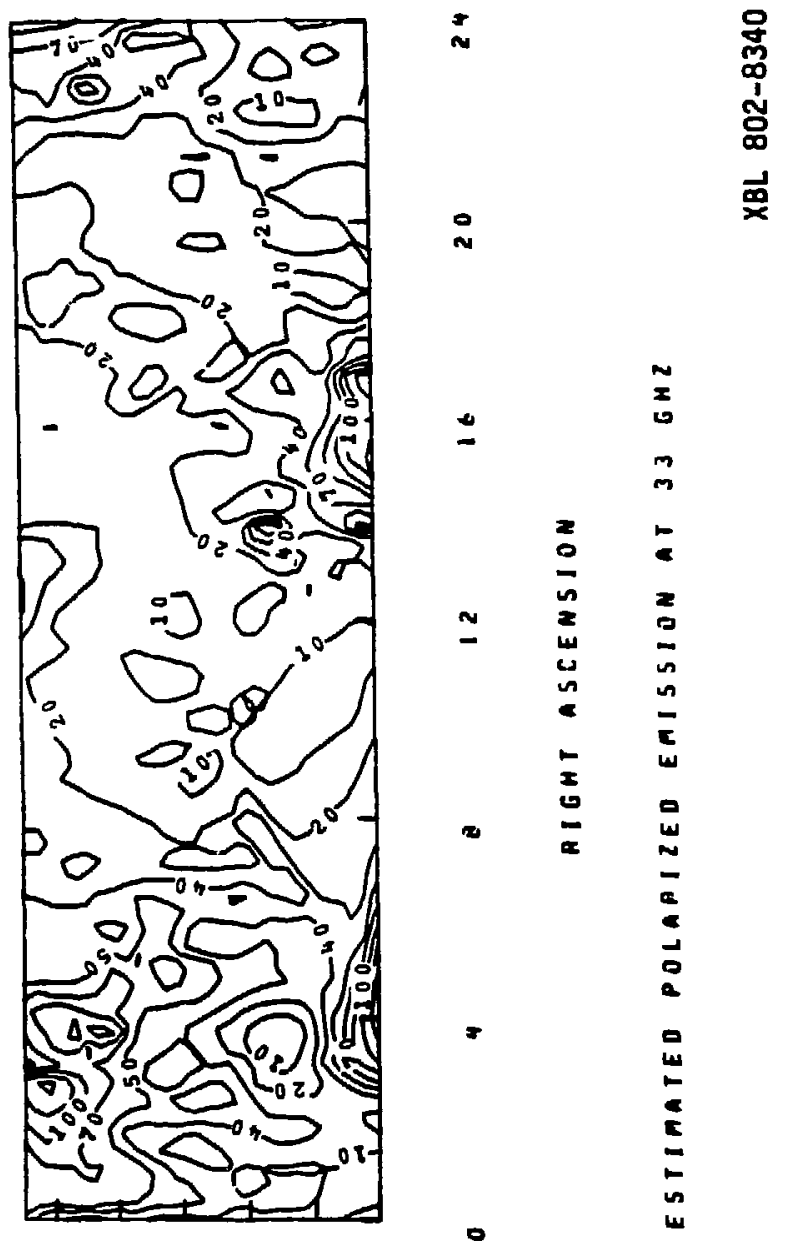

in in un in un un 


\section{D.3 Solar System Sources}

The sun and moon are a potential problem because of their proximity. It is not so much that these bodies emit polarized radiation, but rather that because of small differences in the response pattern of the antenna to differing polarization states a powerful unpolarized source can produce a signal in our system. For this reason data is ignored when these objects are close to the beam axis. The induced signal is less than $0.1 \mathrm{~m}^{\circ} \mathrm{K}$ when the sun is more than $30^{\circ}$ from the beam axis while the corresponding angle for the moon is $20^{\circ}$.

\section{D.3.1 Satellites}

A satellite downlinking at $33 \mathrm{GHz}$ would be a problem. Fortunately, technology has not progressed to this point yet, though in several more years this may not be true. A list of broadcasting sources from ECAC (Electromagnetic Compatibility Analysis Center) shows that we are relatively safe from this type of manmade radialion.

\section{D.3.2 Dust}

Solar system and galactic dust do not produce a significant polarized signal at our frequency of observation. Infared balloon-borne measurements indicate that the total intensity should be well below $0.1 \mathrm{~m}^{\circ} \mathrm{K}$ everywhere except in certain isolated regions near the galactic plane (Owens et al 1979). The polarized component would be substantially less.

\section{D.4 Atmosphere}

The atmusphere has an equivalent antenna temperature of about $T_{A}=12 \%$. Fortunately this is essentially unpolarized. Fog does not appear to be a problem except when it condenses on the rain shield: il!:ils ly for rain collecting on the rain shield. Data taken during these 
times is eliminated. Though scattered sunlight is significantly polarized in the optical, very little is scattered in the microwave because the scattering cross section is inversely proportional to the fourth power of the wavelength (Jackson, 1962).

\section{D.5 Terrestrial Magnetic Fields}

Because the Faraday Rotation Switch (FRS) is inherently magnetically controlled, perturbations in the switching field caused by local fields can be a problem. From knowledge of the switch coil geometry and winding the switch field is approximately $\mathrm{H}=9.2 \mathrm{~g}$. Measurements of the local environment here at Berkeley with a Hall probe magnetometer show the local field is essentially just that of the earth with a total magnitude $0.5 \pm 0.1 \mathrm{~g}$ in agreement with USGS map showing $|\mathbb{B}|=0.51 \mathrm{~g}$ here at Berkeley. Because the ferrite ' 3 the FRS has a magnetization dependent absorption, any external magnetic field combined with a misalignment of the ferrite about the physical rotation axis of the equipment could cause a signal. For this reason the FRS was magnetically shielded with several layers of 4 mil mu-metal foil. Measurements show that

a single layer of u-metal reduces transverse fields by a factor of $10^{2}$ and longitudinal fields by a factor of 10. Tests with a Helmholtz pair (separation equals radius) along three axes at fields up to $10 \mathrm{~B}$ show the induced signal caused by the earth is less than $0.08 \mathrm{~m}^{\circ} \mathrm{K}$ at the $95 \%$ confidence level.

\section{D.6 Depolarization Processes}

Consideration must be given to processes which could reduce or depolarize an initially polarized signal. 


\section{Faraday Rotation}

A plasma in a magnetic field becomes birefringent so a plasma and a turbulent magnetic field could be a possible source of depolarization since this would tend to randomly rotate the polarization. Table D.2 lists the rotation expected from various sources at our frequency. With the exception of an ionized dense universe and a global magnetic field, all known effects are small.

\section{Metric Scrambling}

An interesting geometrical depolarizing effect is that suggested by Brans (1975). Here. an axisymmetric universe causes a scrambling of polarization due to the changing geomerry of the universe. This effect has been shown to be small for "reasonable" universes (Caderni et al. $1978 b)$. 


\section{FARADAY ROTATION \& DEPOLARIZATION}

Plane of polarization rotated by $\Delta \phi \approx 81 \lambda^{2} \int_{N}(r) B(r) \cos \theta(r) d r$

$$
\begin{aligned}
& \lambda-\mathrm{cm}^{-3}-\text { density } \\
& \mathrm{N}-\mathrm{cm}^{-3} \mathrm{e}^{-} \text {deuss } \\
& \mathrm{B}-\text { gaus } \\
& \theta-\text { angle between } \overline{\mathrm{B}} \text { and direction of propagation. } \\
& \mathrm{r}-\mathrm{pc} \quad 1 \mathrm{pc} \sim 3.1 \times 10^{18} \mathrm{~cm} \sim 3.3 \mathrm{ly} \\
& \Delta \phi \simeq 70 \mathrm{NB} \mathrm{r} \quad \lambda=0.91 \mathrm{~cm}
\end{aligned}
$$

Source

Galaxy

lonosphere

Extragalactic ${ }^{1}$

Extragalactic ${ }^{2}$

Solar wind

\begin{tabular}{|l|l|l|l|}
$\mathrm{N} \mathrm{cm}^{-3}$ & $\mathrm{~B} \mathrm{~g}$ & $\mathrm{r} \mathrm{pc}$ & $\Delta \phi \mathrm{rad}$ \\
\hline $10^{-3}$ & $10^{-5}$ & $10^{4}$ & $<10^{-2}$ \\
\hline $10^{6}$ & 1 & $10^{-11}$ & $<10^{-3}$ \\
\hline $10^{-5}$ & $\mathrm{~B}$ & $10^{10}$ & $\leq 10^{7} \mathrm{~B}$ \\
\hline $10^{-9}$ & $\mathrm{~B}$ & $10^{10}$ & $\leq 10^{3} \mathrm{~B}$ \\
\hline 1 & $10^{-4}$ & $10^{-3}$ & $<10^{-5}$ \\
\hline
\end{tabular}

1 Assuming complete ionization in a critically dense universe with a universal magnetic field $B$.

2. As in 1 except ionized fraction $=10^{-4}$. 


\section{Appendix E - Anisotropic Models}

As stated previously, linear polarization of the CBR can result from Thomson scattering of an anisotropic distribution of radiation. This appendix will outline the general approach to the problem of polarization from an anisotropic radiation distribution and discuss in detail the specific model proposed by Rees (1968) of an axisymmetric anisotropic universe.

Following the general method of Chandrasekhar (1950), the equation of transfer for an arbitrary angular distribution and polarization $T(\theta, \phi)$ is given by:

$$
\begin{aligned}
& -\frac{\overline{d l}(\theta, \phi)}{d \tau}=\bar{I}(\theta, \phi)-\bar{J}(\theta, \phi) \\
& \bar{J}(\theta, \phi)=\frac{1}{4 \pi} \int_{\Omega} \bar{P}\left(\theta, \phi, \theta^{\prime}, \phi^{\prime}\right) \bar{I}\left(\theta^{\prime}, \phi^{\prime}\right) d \Omega^{\prime} \\
& \tau-\text { opacity }
\end{aligned}
$$

The first term on the right is the loss due to scattering out of the observation direction while the second term is the increase due to scattering of radiation back into the observation direction. $\bar{P}$ is the called the phase matrix of the scattering and is just the scattering matrix between the initial and final polarization states. The four components of $\vec{T}$ are given below.

$I_{1}$ - Intensity in plane of $Z$ axis

$I_{2}$ - Intensity in $X, Y$ plane

$I_{3}$ - Stokes parameter $U$

$I_{4}$ - Stokes parameter $\mathrm{V}$

Although this notation is different than the usual Stokes parameter notation, it is conenient in scattering problems. The relation between the polarization basis vectors and the physical coordinate system used is shown in Figure E.1. Notice that $Q-I_{1}-I_{2}$ and $I_{\text {toral }}=I_{1}+I_{2}$. As can be seen from (E.1), calculation of the final state of polarization and intensity requires kno" :edge of the initial state and the opacity.

Consider the case of an anisotropically expanding universe, where for simplicity assume there is an axis of symmetry. This is equivalent to assuming the expansion rate proceeds equally rapidly along two axes but at a different rate along the third axis taken to be the $Z$ axis. Conforming to the notation of Rees (1968) the metric describing the expansion is given by: 


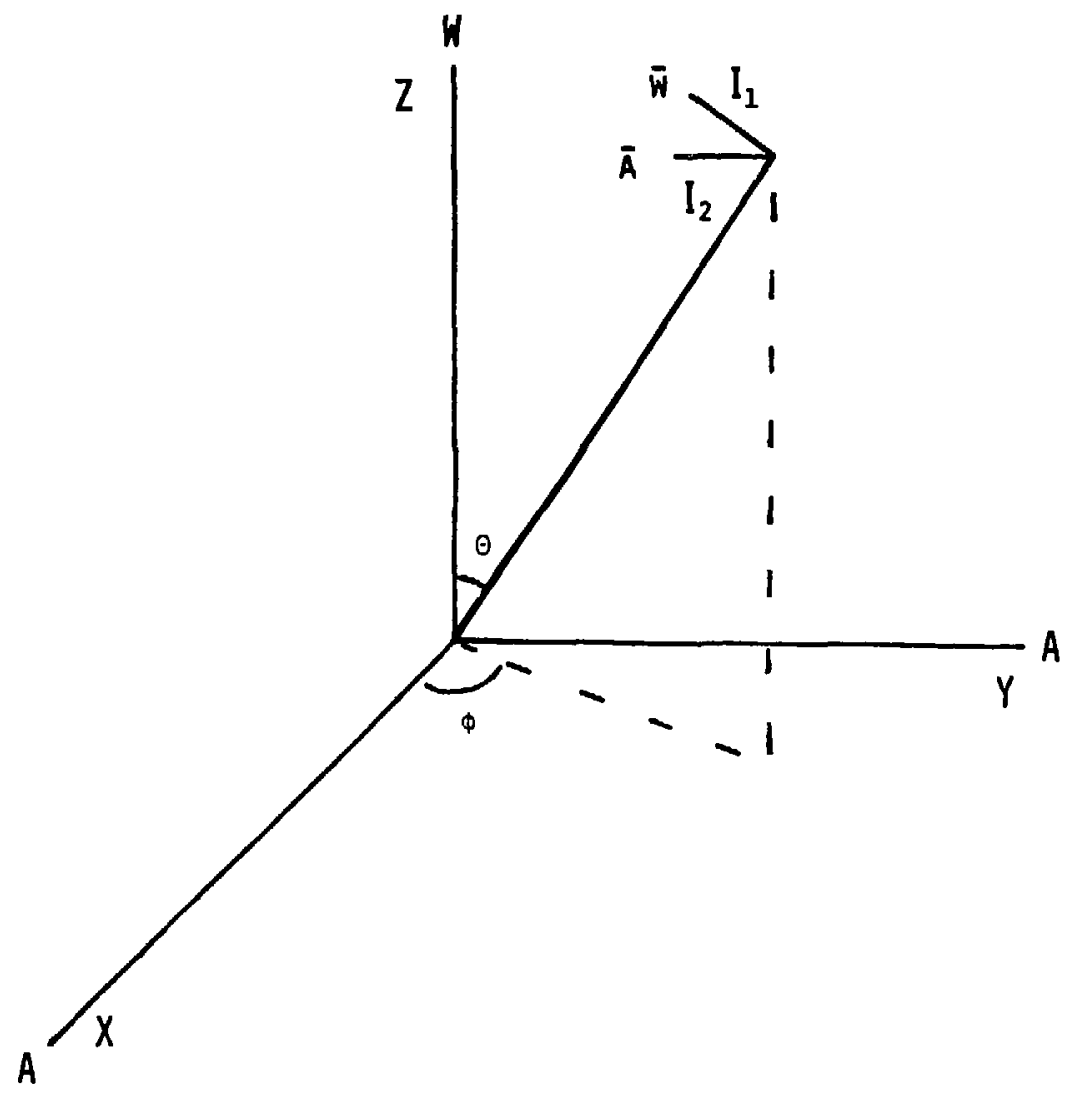

POLARIZATION BASIS VECTORS AND AXISYMMETRIC MODEL COORDINATE SYSTEM

XBL $802-8414$

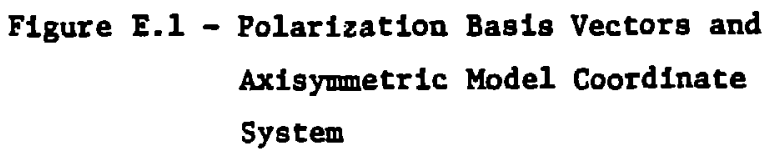




$$
d s^{2}=d t^{2}-A^{2}(1)\left(d x^{2}+d y^{2}\right)-W^{2}(1) d z^{2}
$$

where $A(t)$ and $W(t)$ reflect the different scale sizes of the two expansion rates. The Hubble coefficients for the different directions are defined as:

$$
a(t)=\frac{d A / d t}{A(t)} \quad w(t)=\frac{d W / d t}{W(t)}
$$

The average Hubble coefficient is defined as $h(t)=1 / 3(2 a(t)+w(t))$ while the differential expansion coefficient is defined as $\Delta h(t)=n(t)-a(t)$. As in the standard Big Bang theory, an initial source of blackbody radiation is assumed to exist, which because of the anisotropic expansion is anisotropic in intensity. In the absence of scattering the radiation still has Planckian spectrum but with an effective temperature which has an angle dependence given by

$$
T(1, \theta, \phi)=T(1, \theta, \phi)\left|\frac{A^{2}(1)}{A^{2}\left(l_{1}\right)} \sin ^{2} \theta+\frac{W^{2}(t)}{W^{2}\left(t_{1}\right)} \cos ^{2} \theta\right|^{-1 / 2}
$$

where $t_{\text {, }}$ refers to some "initial" time of interest and $\theta$ is the argle between the observation direction and the $\mathrm{Z}$ axis. For a nearly isotropic universe as ours apparently is, this can be written as:

$$
\begin{aligned}
& T(t, \theta)=T(1,) \frac{W^{2}(t,)}{W^{2}(t)}\left(1+\epsilon\left(1, t_{1}\right) \sin ^{2} \theta\right) \\
& \epsilon\left(1, t_{1}\right)=1 / 2\left(1-\left(\frac{A(t)}{A(t,)} \frac{W(1,)}{W(t)}\right)^{2}\right) \ll 1
\end{aligned}
$$

If scattering is now allowed, the radiation will become partially polarized. To allow for the polarization, the temperature of radiation polarized along the directions $\vec{a}$ and $\overline{\boldsymbol{n}}^{\prime}$ (Figure E.1) can be written as:

$$
\begin{aligned}
& T_{n}(t, \theta)=T(t)\left(1+\epsilon_{n}(t) \sin ^{2} \theta\right) \\
& T_{a}(t, \theta)=T(t)\left(1+\epsilon_{a}(t) \sin ^{2} \theta\right)
\end{aligned}
$$

The equation of transfer can then be written as (Rees 1968, Nanos 1979 , Negroponte and Silk 1980):

$$
\begin{aligned}
& \frac{d}{d t}\left(\begin{array}{l}
\epsilon_{a} \\
\epsilon_{w}
\end{array}\right)=\Delta h\left(\begin{array}{l}
1 \\
1
\end{array}\right)+\frac{1}{\tau}\left(\begin{array}{cc}
-1 & 0 \\
-1 / 2 & -3 / 10
\end{array}\right)\left(\begin{array}{l}
\epsilon_{u} \\
\epsilon_{k}
\end{array}\right) \\
& T=\left(n_{e}(t) \sigma_{T} c\right)^{-1}-\text { mean time between scarterings }
\end{aligned}
$$

The first term on the right represents the effect of anisotropic expansion with no scattering, 
while the second term gives the effect of scattering.

The intensity and polarization anisotropy parameters are defined respectively as:

$$
\begin{aligned}
& \epsilon(t) \equiv 1 / 2\left(\epsilon_{a}(t)+\epsilon_{w}(t)\right) \\
& P(t) \equiv \epsilon_{w}(t)-\epsilon_{a}(t)
\end{aligned}
$$

Negroponte and Silk (1980) have solved the equation of transfer for a number of cases.

The results are shown in Figure E.2. Several general features should be noted.

1) In the absence of reionization after decoupling P/6 -0.04 ror a critically dense universe with all matter jonized before recombination.

2) In the presence of reionization the polarization is very sensitive to the time of reionization and fraction reionized and so could be a sensitive probe of decoupling if an intrinsic anisotropy is found in the CBR.

3) If substantial reionization occurs, polarization and intensity anisotropy can be comparable. Note the case of reheat at $1+z=9$ possibly corresponding to (protol galaxy formation. 
$v_{1} 1=+$ zation (dashed 1ines) and intensity anisotropy (solid lines) in units of present shear to Hubble constant $\left(\Delta H_{0} / H_{0}\right)$ for $T_{0}=2.7^{\circ} \mathrm{K}, h=\Omega=1$ as a function of epoch. $\mathrm{H}_{0}=100 \mathrm{~km} / \mathrm{sec} / \mathrm{tpc}$
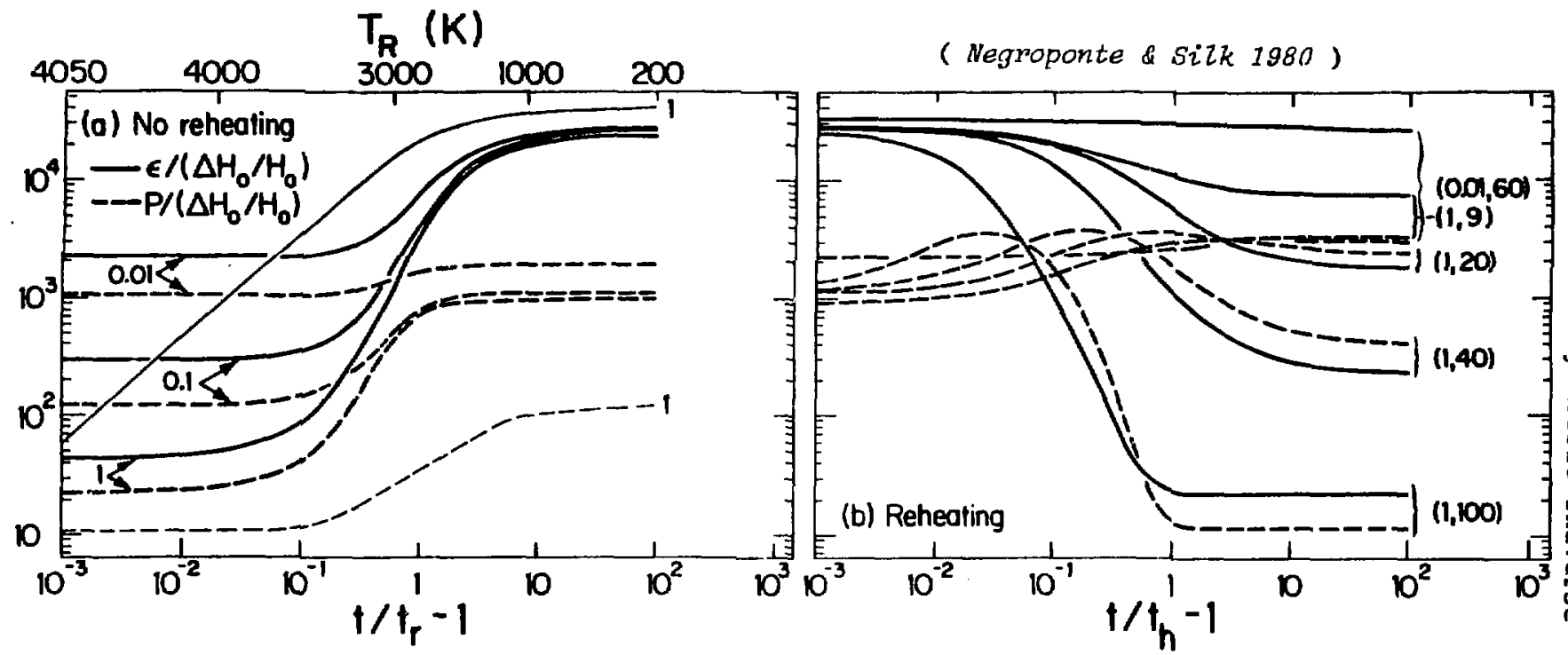

a) Polarization and intensity anisotropy as a function of time after decoupling XEL $\quad$ r $11-13200 \mathrm{C}$ assuming no reheat. The thin lines correspond to the case of instantaneous decoupling while the bold lines use the calculation of Peebles (1968) for the Ionlzation during decoupling. The parameter labeling the lines is the fraction of matter lonized before decoupling while ${ }_{r}$ is the time at decoupling.

b) Polarization and intensity anisotropy as a function of time after reheat. The first and second parameters are the fraction of matter ionized before decoupling and $1+z_{h}\left(z_{h}=2\right.$ at reheat) while $t_{h}$ is the time at reheat. 


\section{Acknowledgments}

As a graduate student it has been my privilege to be associated with a uniquely outstanding and diverse research group. I am particularly grateful for the opportunity to have worked with Andy Buffington, Charles Orth and Terry Mast prior to starting this project. Andy's ability to reduce a problem to its simplest terms and then constantly probe this until it yields a solution has been a valuable experience. Terry's consistent and balanced approach to all types of physics has been a constant source of inspiration to me.

In pursuing this project it has been my fortune to work closely with George Smoot whose constant zeal and enthusiasm for this project and others we have worked on has taught me a great deal. I have especially appreciated the responsibility and freedom he has allowed me. George has been a continual source of insight and knowledge.

I would like to acknowledge the contributions of Rich Muller, Tony Tyson and Luis Alvarez to my education. Rich Muller's creative and novel approach to problems and science in general has left an indelible impression on me. Tony Tyson with whom I regret I could not interact more during his sabbatical stay with our group, showed a true interest in my ideas and encouraged me to pursue them even though I doubted their worth. Luis Alvarez in whom our group is reflected in many ways is an example of how one can seek simplicity in apparent chaos and that through diligence it is possible to "push back the frontiers of knowledge", as he is t und of saying.

This project would not have been fruitful without the support of many others in our group, particularly Hal Dougherty for the mechanical assistance and numerous lunchtime stories, John Gibson and John Yamada for the electronics support, John Aymong for programming assistance, Marsha McBride for a smile every day and eighty words per minute on the typewriter and Lynda Drexler and Nancy: Gusack for the very professional administration of the group. 
l'd like to thank my fellow students ex and otherwise, Marc Gorenstein, Lynne Deutsch. Scott Freidman, Steve Pollaine and Chris Witebsky, better known as Mr. Galactic Background, for their help and support. I am grateful to the United States taxpayers for three years support as a National Science Foundation Fellow. I can only hope someday I'll make enough to pay them back in taxes. The Physics Department here at Berkeley has been extremely supportive in all matters and I would like to acknowledge the true brains behind the department, Teri Doizaki, without whom it would surely perish.

The Lawrence Berkeley Laboratory is a uniquely versatile institution and has created a very productive and pleasurable research environment. The Space Sciences Laboratory under Buford Price has been extraordinarily supportive of this group and has contributed substantially to our ability to carry on research effectively.

Finally l'd like to thank my wife Georganne for putting up with me during the seemingly endless nights, weeks, months 1 was away. She has been an endless source of encouragement for me. Truly the Ph.D. belongs to her for "Putting husband through the Degree."

This work was prepared with the support of the U. S. Department of Energy under Contract W-7405-ENG-48. 


\section{References}

Alpher, R. A., Bethe, H. \&amow, G., Phys. Rev. 73,803 (1948).

Anile, A. M. \& Breuer, R. A., Ap. J. 217,353 (1977).

Berkhuijsen, E. M., Astron. Astrophys. 14, 359 (1971).

Berkhuijsen, E. M., Astron. Astrophys. Suppl. 5 , 263 (1972).

Brans, C. H., Ap. J. 197,1 (1975).

Brouw, W. N. \& Spoelstra, T. A. Th., Astron. A sirophys. Suppl. 26,129 (1976).

Caderni, N., Fabbri, R., Melchiorri, B., Melchiorri, F. \& Natale, V., Phys. Rev. D17 , 8. 1908 (1978a).

Caderni, N., Fabbri, R., Melchiorri, B., Melchiorri, F. \& Natale, V., Phys. Rev. D17 , 8, 1901 (1978b).

Chandrasekhar, S., Radianve Transfer (Clarendon Press, 1950).

Cheng, E. S., Saulson, P. R., Wilkinson, D. T. \& Corey, B. E., Ap. J. Lett. 232 , L139 (1979).

Chu, T. S., Gans, M. J. \& Legg, W. E., Bell. Sys. Tech. J. 54, 10, 1665 (1975).

Collins, C. B. \& Hawking, S. W., Mon. Not. R. Astr. Soc. 162, 307 (1973).

Corey, B. E. \& Wilkinson, D. T., Bull. Am. Astro. Soc. 8 , 351 (1976).

Dicke, R. H., Peebles, P. J. E., Roll, P. G. \& Wilkinson, D. T., Ap. J. 142 , 414 (1965).

Freund, J. E., Mathematical Statistics (Prentice-Hall, 1971, 2nd ed.).

Gorenstein, M. V. \& Smoot, G. F., Ap. J. to be published 1980.

Gurevich, A. G., Ferriles at Microwave Frequencies (Consultants Bureau, New York, 1960).

Harrison, E. R., Ann. Rev. Astron. Astrophys. , 11, 155 (1973).

Hawking, S., Mon. Not. R. Astr. Soc. 142,129 (1969).

Jackson, J. D., Classical Electrodynamics (John Wiley \& Sons, New York, 1962). 
Janssen, M. A., Bednarczyk, S. M., Gulkis, S., Marlin, H. W. \& Smoot, G. F., IEEE Trans. Ant. Prop. AP-27, 4 (1979).

Kraus, J. D.. Radio Astronomy (McGraw-Hill, 1966).

Lubin, P. M. \& Smoot, G. F., Phys. Rev. Lett. 42, 2, 129 (1979).

Mach, E., The Science of Mechanics (Open Court Publishing Co., 1893, 2nd ed.).

Nanos, G. P., Ph.D. Thesis, Princelon University, 1974.

Nanos, G. P., Ap. J. 232,341 (1979).

Negroponte, J. \& Silk, J. Phys. Rev. Lett. to be published (1980).

Owens, D. K., Muehiner, D. J. \& Weiss, R., Ap. J. 231 , 702 (1979).

Peebles, P. J. E., Ap. J. 153,1 (1968).

Peebles, P. J. E., Physical Cosmology (Princeton University Press, Princeton, N.J., 1971).

Penzias. A. A. \& Wilson, R. W., Ap. J. 142.419 (1965).

Rees, M. J., Ap. J. Lett. 153 , Ll (1968).

Shurcliff, W. A. \& Ballard, S. S., Polarized Light (D. Van Nostrand Co., N.J., 1962).

Smoot, G. F., Gorenstein, M. V. \& Muller, R. A., Phys. Rev. Lett. 39, 14, 898 (1977).

Smoot, G. F. \& Lubin, P. M., Ap. J. Letr. 234, L117 (1979).

Taylor, R. E., Proc. IEEE 61 , 4,469 (1973).

Weinberg. S., Gravitation and Cosmology: Principles and Applications of the General Theory of Relativity (John Wiley \& Sons, N.Y., 1972).

Waterman, T. H., Scientific American 193,88 (1955).

Wilson, R. W., Science 205, 31, 866 (1979).

Witebsky, C., Internal group memo, unpublished, NASA note number 361, 1978.

Woody, D. P., Ph.D. Thesis, U.C. Berkeley, 1975.

Woody, D. P. \& Richards, P. L., Phys. Rev. Lett. 42 , 14, 925 (1979).

Yariv, A., Quantum Electronites (John Wiley \& Sons, N.Y., ins?, isi żu.j. 
Zeldovich, Y. B., Kurt, V. G. \&unyaev, R. A., JETP 28,1 (1969). 